\title{
Quantitative uniqueness estimates for the shallow shell system and their application to an inverse problem
}

\author{
Michele Di Cristo, Ching-Lung Lin and Jenn-NAN Wang
}

\begin{abstract}
In this paper we derive some quantitative uniqueness estimates for the shallow shell equations. Our proof relies on appropriate Carleman estimates. For applications, we consider the size estimate inverse problem.
\end{abstract}

Mathematics Subject Classification (2010): 53J58 (primary); 35R30 (secondary).

\section{Introduction}

In this work we study a quantitative uniqueness for the shallow shell system and its application to the inverse problem of estimating the size of an embedded inclusion by boundary measurements. To begin, we let $\Omega$ be a bounded domain in $\mathbb{R}^{2}$. Without loss of generality, we assume $0 \in \Omega$. Let $\bar{\theta}: \bar{\Omega} \rightarrow \mathbb{R}$ satisfy an appropriate regularity assumption which will be specified later. For a shallow shell, its middle surface is described by $\left\{\left(x_{1}, x_{2}, \varepsilon \rho_{0} \bar{\theta}\left(x_{1}, x_{2}\right)\right):\left(x_{1}, x_{2}\right) \in \bar{\Omega}\right\}$ for $\varepsilon>0$, where $\rho_{0}>0$ is the characteristic length of $\Omega$ (see Section 3.1). From now on, we set $\theta=\rho_{0} \bar{\theta}$. Let $u=\left(u_{1}, u_{2}, u_{3}\right)=\left(u^{\prime}, u_{3}\right): \Omega \rightarrow \mathbb{R}^{3}$ represent the displacement vector of the middle surface. Then $u$ satisfies the following equations:

$$
\left\{\begin{array}{l}
-\partial_{j} n_{i j}^{\theta}(u)=0 \quad \text { in } \Omega, \\
\partial_{i j}^{2} m_{i j}\left(u_{3}\right)-\partial_{j}\left(n_{i j}^{\theta}(u) \partial_{i} \theta\right)=0 \quad \text { in } \Omega,
\end{array}\right.
$$

where

$$
\begin{aligned}
m_{i j}\left(u_{3}\right) & =\rho_{0}^{2}\left\{\frac{4 \lambda \mu}{3(\lambda+2 \mu)}\left(\Delta u_{3}\right) \delta_{i j}+\frac{4 \mu}{3} \partial_{i j}^{2} u_{3}\right\}, \\
n_{i j}^{\theta}(u) & =\frac{4 \lambda \mu}{\lambda+2 \mu} e_{k k}^{\theta}(u) \delta_{i j}+4 \mu e_{i j}^{\theta}(u), \\
e_{i j}^{\theta}(u) & =\frac{1}{2}\left(\partial_{i} u_{j}+\partial_{j} u_{i}+\left(\partial_{i} \theta\right) \partial_{j} u_{3}+\left(\partial_{j} \theta\right) \partial_{i} u_{3}\right),
\end{aligned}
$$

Lin and Wang's work was supported in part by the National Science Council of Taiwan.

Received December 2, 2010; accepted May 20, 2011. 
and $\lambda, \mu$ are Lamé coefficients. Hereafter, the Roman indices (except $n$ ) belong to $\{1,2\}$ and the Einstein summation convention is used for repeated indices.

Assume that $D$ is a measurable subdomain of $\Omega$ with $\bar{D} \subset \Omega$. We consider Lamé parameters

$$
\tilde{\lambda}=\lambda+\chi_{D} \lambda_{0} \quad \text { and } \quad \tilde{\mu}=\mu+\chi_{D} \mu_{0}
$$

where $\chi_{D}$ is the characteristic function of $D$. The domain $D$ represents the inclusion inside of $\Omega$. With such parameters $\widetilde{\lambda}, \widetilde{\mu}$, we denote the displacement field $\widetilde{u}=$ $\left(\tilde{u}^{\prime}, \tilde{u}_{3}\right)^{t}$ satisfying (1.1) and the Neumann boundary conditions on $\partial \Omega$ :

$$
\left\{\begin{array}{l}
\tilde{n}_{i j}^{\theta} v_{j}=\rho_{0}^{-1} \widehat{T}_{i} \\
\tilde{m}_{i j} v_{i} v_{j}=\widehat{M}_{\nu} \\
\left(\partial_{i} \tilde{m}_{i j}-\tilde{n}_{i j}^{\theta} \partial_{i} \theta\right) v_{j}+\partial_{s}\left(\widetilde{m}_{i j} v_{i} \tau_{j}\right)=-\partial_{s} \widehat{M}_{\tau}
\end{array}\right.
$$

where $\tilde{m}_{i j}=\tilde{m}_{i j}\left(\tilde{u}_{3}\right)$ and $\tilde{n}_{i j}^{\theta}=\tilde{n}_{i j}^{\theta}(\widetilde{u})$ are defined in (1.2) with $\lambda, \mu, u$ replaced by $\tilde{\lambda}, \tilde{\mu}, \tilde{u}$. Hereafter, $v=\left(v_{1}, v_{2}\right), \tau=\left(\tau_{1}, \tau_{2}\right)$ are, respectively, the normal and the tangent vectors along $\partial \Omega$, and $s$ is the arclength parameter of $\partial \Omega$. Precisely, the tangent vector $\tau$ is obtained by rotating $v$ counterclockwise of angle $\pi / 2$. The boundary field $\widehat{M}=\widehat{M}_{\tau} \nu+\widehat{M}_{\nu} \tau$, i.e., $\widehat{M}_{\tau}=\widehat{M} \cdot v$ and $\widehat{M}_{\nu}=\widehat{M} \cdot \tau$. We remark that in the plate theory, $\widehat{M}_{\tau}$ and $\widehat{M}_{\nu}$ are the twisting and bending moments applied on $\partial \Omega$. The field $\widehat{T}$ satisfies the compatibility condition which will be specified in the following section. An interesting inverse problem is to determine geometric information on $D$ from a pair $\left\{\widehat{T}, \widehat{M} ;\left.\widetilde{u}^{\prime}\right|_{\partial \Omega},\left(\left.\widetilde{u}_{3}\right|_{\partial \Omega},\left.\partial_{\nu} \widetilde{u}_{3}\right|_{\partial \Omega}\right)\right\}$, i.e., from the Cauchy data of the solution $\tilde{u}$. Despite its practical value, the fundamental global uniqueness, even for the scalar equation, is yet to be proved. For the development of the uniqueness issue for this kind of inverse problems, we refer to [13] and references therein for details.

In this paper we are interested in estimating the size of the area of $D$ in terms of the Cauchy data of $\tilde{u}$. This type of problem has been studied for the scalar equation and for systems of equations such as the isotropic elasticity and plate. We refer to the survey article [3] for the early developments and [20,21] for the latest results on the plate equations. Specifically, the size of $D$ is estimated by the following two quantities:

$$
\widetilde{W}=\int_{\partial \Omega} \rho_{0}^{-1} \widehat{T} \cdot \tilde{u}^{\prime}+\widehat{M}_{\nu} \partial_{\nu} \tilde{u}_{3}+\partial_{s} \widehat{M}_{\tau} \widetilde{u}_{3}
$$

and

$$
W=\int_{\partial \Omega} \rho_{0}^{-1} \widehat{T} \cdot u^{\prime}+\widehat{M}_{\nu} \partial_{\nu} u_{3}+\partial_{s} \widehat{M}_{\tau} u_{3},
$$

where $u=\left(u^{\prime}, u_{3}\right)^{t}$ is the displacement vector satisfing (1.1) and (1.3) with $D=\emptyset$, i.e., $\tilde{\lambda}=\lambda$ and $\tilde{\mu}=\mu$. Here we assume that $\lambda, \mu$ are given a priori, thus, both $\widetilde{W}$ and $W$ are known. To be more precise, in this paper, we will show that under some 
a priori assumptions, there exist positive constants $C_{1}, C_{2}$ such that

$$
C_{1}\left|\frac{\tilde{W}-W}{W}\right| \leq \operatorname{area}(D) \leq C_{2}\left|\frac{\widetilde{W}-W}{W}\right|
$$

where $C_{1}, C_{2}$ depend on the a priori data.

The derivation of the volume bounds on $D$ relies on the following integral inequalities

$$
\begin{aligned}
\frac{1}{K} \int_{D} \sum_{i j}\left|e_{i j}^{\theta}(u)\right|^{2}+\rho_{0}^{2}\left|\partial_{i j}^{2} u_{3}\right|^{2} & \leq|W-\widetilde{W}| \\
& \leq K \int_{D} \sum_{i j}\left|e_{i j}^{\theta}(u)\right|^{2}+\rho_{0}^{2}\left|\partial_{i j}^{2} u_{3}\right|^{2},
\end{aligned}
$$

where the constant $K$ depends on the a priori data. The lower bound for area $(D)$ is a consequence of the second inequality of (1.5) and the elliptic regularity estimate for $u$. To derive the upper bound for area $(D)$, we shall use the first inequality of (1.5). As indicated in all previous related results, we need to estimate $\int_{D} \sum_{i j}\left|e_{i j}^{\theta}(u)\right|^{2}+\rho_{0}^{2}\left|\partial_{i j}^{2} u_{3}\right|^{2}$ from below. This can be achieved by the quantitative uniqueness estimates of solutions $u$ solving (1.1), which is one of the themes of the paper.

For the second order elliptic operator, using the Carleman or the frequency functions methods, quantitative estimates for the strong unique continuation under different assumptions on coefficients were derived in $[8-11,14,16,18]$. For the isotropic elasticity, similar estimates can be found in $[1,4,19]$. Further, for the elastic plate, quantitative uniqueness estimates were derived in [20,21]. Note that global versions of quantitative uniqueness estimates, in the form of doubling inequality, were given in [4] and [21], where their arguments rely on a local version for the power of Laplacian derived in [17].

In this paper, we will derive three-ball inequalities and doubling inequalities for the shallow shell system (1.1) with $\lambda, \mu \in C^{1,1}(\Omega)$. Since the first and the second equations in (1.1) have different orders, it seems that the Carleman method is the most efficient way to derive those quantitative uniqueness estimates for (1.1). We will give detailed derivations of quantitative uniqueness estimates based on the Carleman estimates in Section 4. The investigation of the inverse problem is given in Section 5. Since the Neumann boundary value problem for (1.1) is not standard, we will first study this forward problem in Section 3.

\section{Notation}

Definition 2.1. Let $\Omega$ be a bounded domain in $\mathbb{R}^{n}$ with $n \geq 2$. Given $k \in \mathbb{Z}^{+}$, we say that $\partial \Omega$ is of class $C^{k, 1}$ with constants $\rho_{0}, A_{0}$, if, for any point $z \in \partial \Omega$, there 
exists a rigid coordinate transformation under which $z=0$ and

$$
\Omega \cap B_{\rho_{0}}(0)=\left\{x=\left(x_{1}, \cdots, x_{n-1}, x_{n}\right)=\left(x^{\prime}, x_{n}\right) \in B_{\rho_{0}}(0): x_{n}>\varphi\left(x^{\prime}\right)\right\},
$$

where $\varphi\left(x^{\prime}\right)$ is a $C^{k, 1}$ function on $B_{\rho_{0}}^{\prime}(0)=B_{\rho_{0}}(0) \cap\left\{x_{n}=0\right\}$ satisfying $\varphi(0)=0$ and $\nabla \varphi(0)=0$ if $k \geq 1$ and

$$
\|\varphi\|_{C^{k, 1}\left(B_{\rho_{0}}^{\prime}(0)\right)} \leq A_{0} \rho_{0} .
$$

Throughout the paper, we will normalize all norms such that they are dimensionally homogeneous and coincide with the standard definitions when the dimensional parameter is one. With this in mind, we define

$$
\|\varphi\|_{C^{k, 1}\left(B_{\rho_{0}}^{\prime}(0)\right)}=\sum_{j=0}^{k} \rho_{0}^{j}\left\|\nabla^{j} \varphi\right\|_{L^{\infty}\left(B_{\rho_{0}}^{\prime}(0)\right)}+\rho_{0}^{k+1}\left\|\nabla^{k+1} \varphi\right\|_{L^{\infty}\left(B_{\rho_{0}}^{\prime}(0)\right)} .
$$

Similarly, when $\Omega$ with $\partial \Omega$ defined above and $w: \Omega \rightarrow \mathbb{R}$, we define

$$
\begin{aligned}
\|w\|_{C^{k, 1}(\Omega)} & =\sum_{j=0}^{k} \rho_{0}^{j}\left\|\nabla^{j} w\right\|_{L^{\infty}(\Omega)}+\rho_{0}^{k+1}\left\|\nabla^{k+1} w\right\|_{L^{\infty}(\Omega)}, \\
\|w\|_{L^{2}(\Omega)}^{2} & =\rho_{0}^{-n} \int_{\Omega} w^{2}, \\
\|w\|_{H^{k}(\Omega)}^{2} & =\rho_{0}^{-n} \sum_{j=0}^{k} \rho_{0}^{2 j} \int_{\Omega}\left|\nabla^{j} w\right|^{2}, \quad k \geq 1 .
\end{aligned}
$$

In particular, if $\Omega=B_{\rho}(0)$, then $\Omega$ satisfies Definition 2.1 with $\rho_{0}=\rho$.

Let $\mathcal{A}$ be an open connected component of $\partial \Omega$. For any given point $z_{0} \in \mathcal{A}$, we define the positive orientation of $\mathcal{A}$ associated with an arclength parametrization $\zeta(s)=\left(x_{1}(s), x_{2}(s)\right), s \in[0, \operatorname{length}(\mathcal{A})]$ such that $\zeta(0)=z_{0}$ and $\zeta^{\prime}(s)=\tau(\zeta(s))$. Finally, we define for any $h>0$

$$
\Omega_{h}=\{x \in \Omega \mid \operatorname{dist}(x, \partial \Omega)>h\} .
$$

\section{The forward problem}

\subsection{The Neumann boundary value problem for the shallow shell equation}

At this moment, we assume $\partial \Omega \in C^{1,1}$ with constants $A_{0}, \rho_{0}$. Also, let $\Omega$ satisfy

$$
|\Omega| \leq A_{1} \rho_{0}^{2}
$$

throughout the article, and

$$
\|\nabla \theta\|_{L^{\infty}(\Omega)}=\rho_{0}\|\nabla \bar{\theta}\|_{L^{\infty}(\Omega)} \leq A_{2}
$$


for some positive constants $A_{1}$ and $A_{2}$. We will investigate the Neumann boundary value problem, the forward problem, for the shallow shell system. To begin, let us assume that Lamé coefficients $\lambda, \mu \in L^{\infty}(\Omega)$ satisfying

$$
0<\delta_{0} \leq \mu(x), \quad \delta_{0} \leq \lambda(x), \quad \forall x \in \Omega .
$$

We aim to find $u=\left(u_{1}, u_{2}, u_{3}\right)=\left(u^{\prime}, u_{3}\right)$ satisfying

$$
\left\{\begin{array}{l}
-\partial_{j} n_{i j}^{\theta}(u)=0 \quad \text { in } \Omega, \\
\partial_{i j}^{2} m_{i j}\left(u_{3}\right)-\partial_{j}\left(n_{i j}^{\theta}(u) \partial_{i} \theta\right)=0 \quad \text { in } \Omega,
\end{array}\right.
$$

with boundary conditions

$$
\left\{\begin{array}{l}
n_{i j}^{\theta}(u) v_{j}=\rho_{0}^{-1} \widehat{T}_{i}, \\
m_{i j}\left(u_{3}\right) v_{i} v_{j}=\widehat{M}_{\nu}, \\
\left(\partial_{i} m_{i j}\left(u_{3}\right)-n_{i j}^{\theta}(u) \partial_{i} \theta\right) v_{j}+\partial_{s}\left(m_{i j}\left(u_{3}\right) v_{i} \tau_{j}\right)=-\partial_{s} \widehat{M}_{\tau} .
\end{array}\right.
$$

Now assume that $u=\left(u^{\prime}, u_{3}\right)$ satisfies (3.4)-(3.5). Let $v=\left(v^{\prime}, v_{3}\right) \in\left(H^{1}(\Omega)\right)^{2} \times$ $H^{2}(\Omega)$, then multiplying the first and second equations of (3.4) by $v^{\prime}$ and $v_{3}$, respectively, and using the standard integration by parts, we can obtain that

$$
\int_{\Omega} \sum_{i j}\left(n_{i j}^{\theta}(u) e_{i j}^{\theta}(v)+m_{i j}\left(u_{3}\right) \partial_{i j}^{2} v_{3}\right)=\int_{\partial \Omega} \rho_{0}^{-1} \widehat{T} \cdot v^{\prime}+\partial_{s} \widehat{M}_{\tau} v_{3}+\widehat{M}_{\nu} \partial_{\nu} v_{3}
$$

The boundary field $\widehat{M}=\widehat{M}_{\tau} v+\widehat{M}_{\nu} \tau$ in the cartesian coordinates is written as

$$
\widehat{M}=\widehat{M}_{1} e_{2}+\widehat{M}_{2} e_{1} .
$$

In view of the relation

$$
\partial_{s} \widehat{M}_{\tau} v_{3}=\partial_{s}\left(\widehat{M}_{\tau} v_{3}\right)-\widehat{M}_{\tau} \partial_{s} v_{3},
$$

one can see that the right-hand side of (3.6) becomes

$$
\int_{\partial \Omega} \rho_{0}^{-1} \widehat{T} \cdot v^{\prime}-\widehat{M}_{\tau} \partial_{s} v_{3}+\widehat{M}_{\nu} \partial_{\nu} v_{3}
$$

Recall that $\partial_{j} v_{3}=\partial_{s} v_{3} \tau_{j}+\partial_{\nu} v_{3} v_{j}$ for $j=1,2$. Using the relation $\tau=\left(-v_{2}, v_{1}\right)$ if $v=\left(v_{1}, v_{2}\right)$, we get that

$$
\begin{aligned}
\widehat{M}_{1} \partial_{1} v_{3}-\widehat{M}_{2} \partial_{2} v_{3} & =\widehat{M}_{1}\left(\partial_{s} v_{3} \tau_{1}+\partial_{\nu} v_{3} v_{1}\right)-\widehat{M}_{2}\left(\partial_{s} v_{3} \tau_{2}+\partial_{\nu} v_{3} v_{2}\right) \\
& =\left(\widehat{M}_{1} \tau_{1}-\widehat{M}_{2} \tau_{2}\right) \partial_{s} v_{3}+\left(\widehat{M}_{1} v_{1}-\widehat{M}_{2} v_{2}\right) \partial_{\nu} v_{3} \\
& =-\widehat{M}_{\tau} \partial_{s} v_{3}+\widehat{M}_{\nu} \partial_{\nu} v_{3}
\end{aligned}
$$


In view of the above computations, we deduce that

$$
\int_{\Omega} \sum_{i j}\left(n_{i j}^{\theta}(u) e_{i j}^{\theta}(v)+m_{i j}\left(u_{3}\right) \partial_{i j}^{2} v_{3}\right)=\int_{\partial \Omega} \rho_{0}^{-1} \widehat{T} \cdot v^{\prime}+\widehat{M}_{1} \partial_{1} v_{3}-\widehat{M}_{2} \partial_{2} v_{3}
$$

(see the similar derivation for the plate equation in [20]). Let $v^{\prime}=a+W \cdot x+b \theta$ and $v_{3}=c-b \cdot x$, where $a=\left(a_{1}, a_{2}\right), b=\left(b_{1}, b_{2}\right)$ are two-dimensional vectors, $W$ is a $2 \times 2$ skew-symmetric matrix, and $c$ is a scalar. Then $e_{i j}^{\theta}(v)=\partial_{i j}^{2} v_{3}=0$ for all $i, j$. Thus, to solve (3.4) and (3.5), the pair $(\widehat{T}, \widehat{M})$ must satisfy the compatibility condition

$$
\int_{\partial \Omega} \rho_{0}^{-1} \widehat{T} \cdot(a+W \cdot x+b \theta)-b_{1} \widehat{M}_{1}+b_{2} \widehat{M}_{2}=0 .
$$

Note that taking $b=0$, we have the usual compatibility condition for the traction of the elasticity equation, i.e.,

$$
\int_{\partial \Omega} \widehat{T} \cdot(a+W \cdot x)=0
$$

On the other hand, to guarantee uniqueness for the forward problem, we impose the following normalization conditions

$$
\int_{\Omega} u=0, \quad \int_{\Omega} \nabla u_{3}=0, \quad \int_{\Omega}\left(\partial_{1} u_{2}-\partial_{2} u_{1}\right)+\left(\partial_{1} \theta \partial_{2} u_{3}-\partial_{2} \theta \partial_{1} u_{3}\right)=0 .
$$

To solve the forward problem, the following Poincaré-Korn inequality is very important.

Proposition 3.1. There exists an absolute constant $C>0$, depending on $A_{0}, A_{1}, A_{2}$, such that for all $u=\left(u^{\prime}, u_{3}\right) \in\left(H^{1}(\Omega)\right)^{2} \times H^{2}(\Omega)$ satisfying (3.9) we have

$$
\left\|u^{\prime}\right\|_{H^{1}(\Omega)}^{2}+\left\|u_{3}\right\|_{H^{2}(\Omega)}^{2} \leq C \int_{\Omega} \sum_{i j}\left|e_{i j}^{\theta}(u)\right|^{2}+\rho_{0}^{2}\left|\partial_{i j}^{2} u_{3}\right|^{2} .
$$

Proof. The inequality (3.10) is a combination of Poincaré's and Korn's inequalities. By abuse of notation, the variable $x$ in our proof stands for $\left(x_{1}, x_{2}, x_{3}\right)=\left(x^{\prime}, x_{3}\right)$. Now let $\Gamma=\Omega \times\left(-\rho_{0}, \rho_{0}\right) \subset \mathbb{R}^{3}$ and introduce new variables $\tilde{x}^{\prime}=x^{\prime}$ and $\tilde{x}_{3}=x_{3}+\theta\left(x^{\prime}\right)$. Denote by $\widetilde{\Gamma}$ the domain of $\Omega$ under the coordinate transformation $x \mapsto \tilde{x}$, i.e., $\widetilde{\Gamma}=\Omega \times\left(-\rho_{0}+\theta, \rho_{0}+\theta\right)$. Both domains $\Gamma$ and $\widetilde{\Gamma}$ are clearly Lipschitz. On $\widetilde{\Gamma}$, we have the standard Korn's inequality: there exists a constant $K_{0}>0$ such that for any 3 vector $v \in H^{1}(\widetilde{\Gamma})$ satisfying

$$
\int_{\widetilde{\Gamma}} v d \widetilde{x}=0, \quad \int_{\widetilde{\Gamma}}\left(\nabla_{\tilde{x}} v-\left(\nabla_{\tilde{x}} v\right)^{T}\right) d \widetilde{x}=0
$$

we have

$$
\rho_{0}^{-2}\|v\|_{L^{2}(\widetilde{\Gamma})}^{2}+\left\|\nabla_{\tilde{x}} v\right\|_{L^{2}(\widetilde{\Gamma})}^{2} \leq K_{0}\left\|\widehat{\nabla}_{\widetilde{x}} v\right\|_{L^{2}(\widetilde{\Gamma})}^{2},
$$


where $\widehat{\nabla}_{\widetilde{x}} v=\left(\nabla_{\widetilde{x}} v+\left(\nabla_{\tilde{x}} v\right)^{t}\right) / 2$ and $K_{0}$ depends on $A_{0}, A_{1}, A_{2}$. Let $w(x)=$ $w\left(x_{1}, x_{2}, x_{3}\right) \in H^{1}(\Gamma)$, then $v(\tilde{x}):=w\left(\tilde{x}_{1}, \tilde{x}_{2}, \tilde{x}_{3}-\theta\left(\tilde{x}^{\prime}\right)\right) \in H^{1}(\widetilde{\Gamma})$. By observing that the Jacobian of the coordinate transformation $x \mapsto \tilde{x}$ is 1 , we can write (3.11), (3.12) in terms of $x$ and get that for all $w \in H^{1}(\Gamma)$ satisfying

$$
\left\{\begin{array}{l}
\int_{\Gamma} w d x=0 \\
\int_{\Gamma}\left(\partial_{1} w_{2}-\partial_{1} \theta \partial_{3} w_{2}-\partial_{2} w_{1}+\partial_{2} \theta \partial_{3} w_{1}\right) d x=0 \\
\int_{\Gamma}\left(\partial_{1} w_{3}-\partial_{1} \theta \partial_{3} w_{3}-\partial_{3} w_{1}\right) d x=0 \\
\int_{\Gamma}\left(\partial_{2} w_{3}-\partial_{2} \theta \partial_{3} w_{3}-\partial_{3} w_{2}\right) d x=0
\end{array}\right.
$$

we have

$$
\rho_{0}^{-2}\|w\|_{L^{2}(\Gamma)}^{2}+\left\|\nabla_{x}^{\theta} w\right\|_{L^{2}(\Gamma)}^{2} \leq K_{0}\left\|\widehat{\nabla}_{x}^{\theta} w\right\|_{L^{2}(\Gamma)}^{2},
$$

where

$$
\nabla_{x}^{\theta} w=\left(\begin{array}{ccc}
\left(\partial_{1}-\partial_{1} \theta \partial_{3}\right) w_{1} & \left(\partial_{1}-\partial_{1} \theta \partial_{3}\right) w_{2} & \left(\partial_{1}-\partial_{1} \theta \partial_{3}\right) w_{3} \\
\left(\partial_{2}-\partial_{2} \theta \partial_{3}\right) w_{1} & \left(\partial_{2}-\partial_{2} \theta \partial_{3}\right) w_{2} & \left(\partial_{2}-\partial_{2} \theta \partial_{3}\right) w_{3} \\
\partial_{3} w_{1} & \partial_{3} w_{2} & \partial_{3} w_{3}
\end{array}\right)
$$

and the symmetric part $\widehat{\nabla}_{x}^{\theta} w$ of $\nabla_{x}^{\theta} w$ is defined similarly. In fact, by the form of $\nabla_{x}^{\theta} w,(3.14)$ can be improved to

$$
\rho_{0}^{-2}\|w\|_{L^{2}(\Gamma)}^{2}+\left\|\nabla_{x} w\right\|_{L^{2}(\Gamma)}^{2} \leq K_{1}\left\|\widehat{\nabla}_{x}^{\theta} w\right\|_{L^{2}(\Gamma)}^{2}
$$

for some constant $K_{1}$, also depending on $A_{0}, A_{1}, A_{2}$. Now let $u=\left(u^{\prime}, u_{3}\right)^{t} \in$ $\left(H^{1}(\Omega)\right)^{2} \times H^{2}(\Omega)$, we apply (3.13) and (3.15) to

$$
w(x)=\left(u_{1}\left(x^{\prime}\right)-x_{3} \partial_{1} u_{3}\left(x^{\prime}\right), u_{2}\left(x^{\prime}\right)-x_{3} \partial_{2} u_{3}\left(x^{\prime}\right), u_{3}\left(x^{\prime}\right)\right)
$$

where $\left(x^{\prime}, x_{3}\right) \in \Omega \times\left(-\rho_{0}, \rho_{0}\right)$. It is easy to check that the constraints (3.13) are reduced to the normalization conditions (3.9). On the other hand, easy computations show that (3.15) becomes

$\rho_{0} \int_{\Omega}\left(\rho_{0}^{-2}|u|^{2}+|\nabla u|^{2}+\rho_{0}^{2} \sum_{i j}\left|\partial_{i j}^{2} u_{3}\right|^{2}\right) \leq C \int_{\Omega} \sum_{i j}\left(\rho_{0}\left|e_{i j}^{\theta}(u)\right|^{2}+\rho_{0}^{3}\left|\partial_{i j}^{2} u_{3}\right|^{2}\right)$

with $C$ only depending on $A_{0}, A_{1}, A_{2}$.

\subsection{Existence and uniqueness}

We will use the variational method to solve the forward problem. This seems to be standard. But we could not find any literature discussing the Neumann boundary value problem for the shallow shell. For the sake of completeness, we give a proof 
of this forward problem. The arguments used here are adapted from [20]. To begin, let us introduce

$$
\begin{aligned}
H(u, v) & =\int_{\Omega} \sum_{i j} n_{i j}^{\theta}(u) \partial_{j} v_{i}+m_{i j}\left(u_{3}\right) \partial_{i j}^{2} v_{3}+n_{i j}^{\theta}(u) \partial_{i} \theta \partial_{j} v_{3} \\
& =\int_{\Omega} \sum_{i j} n_{i j}^{\theta}(u) e_{i j}^{\theta}(v)+m_{i j}\left(u_{3}\right) \partial_{i j}^{2} v_{3}
\end{aligned}
$$

and

$$
L(v)=\int_{\partial \Omega} \rho_{0}^{-1} \widehat{T} \cdot v^{\prime}+\partial_{s} \widehat{M}_{\tau} v_{3}+\widehat{M}_{\nu} \partial_{\nu} v_{3} .
$$

We now give a weak formulation of the Neumann boundary value problem (3.4)(3.5).

Definition 3.2. A vector valued function $u=\left(u^{\prime}, u_{3}\right)^{t} \in\left(H^{1}(\Omega)\right)^{2} \times H^{2}(\Omega)$ is a weak solution to (3.4)-(3.5) if and only if

$$
H(u, v)=L(v) \text { for all } v=\left(v^{\prime}, v_{3}\right)^{t} \in H^{1}(\Omega) \times H^{2}(\Omega) .
$$

From the above computations, we know that

$$
L(v)=\int_{\partial \Omega} \rho_{0}^{-1} \widehat{T} \cdot v^{\prime}+\widehat{M}_{1} \partial_{1} v_{3}-\widehat{M}_{2} \partial_{2} v_{3}:=\widetilde{L}(v) .
$$

In other words, (3.16) is equivalent to

$$
H(u, v)=\widetilde{L}(v) \text { for all } v=\left(v^{\prime}, v_{3}\right)^{t} \in\left(H^{1}(\Omega)\right)^{2} \times H^{2}(\Omega) .
$$

Theorem 3.3. Assume that $\theta$ satisfies (3.2) and $\lambda, \mu \in L^{\infty}(\Omega)$ satisfy (3.3). Given any boundary field $(\widehat{T}, \widehat{M}) \in H^{-1 / 2}(\partial \Omega)$ and the compatibility condition (3.8) holds. Then (3.4)-(3.5) admits a unique weak solution $u=\left(u^{\prime}, u_{3}\right)^{t}$ satisfying the conditions (3.9) and

$$
\left\|u^{\prime}\right\|_{H^{1}(\Omega)}+\left\|u_{3}\right\|_{H^{2}(\Omega)} \leq C\|(\widehat{T}, \widehat{M})\|_{\left(H^{-1 / 2}(\partial \Omega)\right)^{3}},
$$

where $C$ depends on $A_{0}, A_{1}, A_{2}, \delta_{0}$.

Proof. Let $V$ be the subspace of $\left(H^{1}(\Omega)\right)^{2} \times H^{2}(\Omega)$ characterized by

$$
V=\left\{w=\left(w^{\prime}, w_{3}\right) \in\left(H^{1}(\Omega)\right)^{2} \times H^{2}(\Omega): w \text { satisfies (3.9) }\right\} .
$$

In view of (3.10), we have that

$$
\begin{aligned}
\int_{\Omega} \sum_{i j}\left|e_{i j}^{\theta}(u)\right|^{2}+\rho_{0}^{2}\left|\partial_{i j}^{2} u_{3}\right|^{2} & \leq\left\|u^{\prime}\right\|_{H^{1}(\Omega)}^{2}+\left\|u_{3}\right\|_{H^{2}(\Omega)}^{2} \\
& \leq C \int_{\Omega} \sum_{i j}\left|e_{i j}^{\theta}(u)\right|^{2}+\rho_{0}^{2}\left|\partial_{i j}^{2} u_{3}\right|^{2}
\end{aligned}
$$


for all $u \in V$. We now define a functional $J: V \rightarrow \mathbb{R}$ by

$$
J(u)=\frac{1}{2} H(u, u)-\tilde{L}(u) .
$$

We first want to prove that $J$ has a unique minimizer on $V$. To this end, it suffices to show that $J$ is coercive and strictly convex on $V$. It is easy to see that

$$
\begin{aligned}
H(u, u)= & \int_{\Omega} n_{i j}^{\theta}(u) e_{i j}^{\theta}(u)+m_{i j}\left(u_{3}\right) \partial_{i j}^{2} u_{3} \\
= & \int_{\Omega} \frac{4 \lambda \mu}{\lambda+2 \mu}\left|\sum_{k} e_{k k}^{\theta}(u)\right|^{2}+4 \mu \sum_{i j}\left|e_{i j}^{\theta}(u)\right|^{2} \\
& \quad+\frac{4 \lambda \mu \rho_{0}^{2}}{3(\lambda+2 \mu)}\left|\Delta u_{3}\right|^{2}+\frac{4 \mu \rho_{0}^{2}}{3} \sum_{i j}\left|\partial_{i j}^{2} u_{3}\right|^{2} \\
\geq & \frac{4 \delta_{0}}{3} \int_{\Omega} \sum_{i j}\left|e_{i j}^{\theta}(u)\right|^{2}+\rho_{0}^{2}\left|\partial_{i j}^{2} u_{3}\right|^{2} .
\end{aligned}
$$

Thus, (3.19) implies

$$
H(u, u) \geq C\left(\left\|u^{\prime}\right\|_{H^{1}(\Omega)}^{2}+\left\|u_{3}\right\|_{H^{2}(\Omega)}^{2}\right)
$$

with $C$ depending only on $A_{0}, A_{1}, A_{2}, \delta_{0}$. On the other hand, the trace inequality leads to

$$
\widetilde{L}(u) \leq C\|(\widehat{T}, \widehat{M})\|_{\left(H^{-1 / 2}(\partial \Omega)\right)^{3}}\left(\left\|u^{\prime}\right\|_{H^{1}(\Omega)}+\left\|u_{3}\right\|_{H^{2}(\Omega)}\right) .
$$

Consequently, we obtain that

$$
\begin{aligned}
J(u) \geq C\left(\left\|u^{\prime}\right\|_{H^{1}(\Omega)}^{2}\right. & +\left\|u_{3}\right\|_{H^{2}(\Omega)}^{2} \\
& \left.\quad\|(\widehat{T}, \widehat{M})\|_{\left(H^{-1 / 2}(\partial \Omega)\right)^{3}}\left(\left\|u^{\prime}\right\|_{H^{1}(\Omega)}+\left\|u_{3}\right\|_{H^{2}(\Omega)}\right)\right),
\end{aligned}
$$

which shows that $J$ is coercive and bounded from below on $V$.

Now for $t \in[0,1]$ and $u, v \in V$, we have that

$$
\begin{aligned}
& H(t u+(1-t) v, t u+(1-t) v)-t H(u, u)-(1-t) H(v, v) \\
= & -t(1-t) H(u-v, u-v) \leq 0
\end{aligned}
$$

and for $t \in(0,1)$

$$
H(t u+(1-t) v, t u+(1-t) v)=t H(u, u)+(1-t) H(v, v)
$$

if and only if

$$
H(u-v, u-v)=0 .
$$


Since $u, v \in V$, we see that $H(u-v, u-v)=0$ if and only if $u=v$ in $\left(H^{1}(\Omega)\right)^{2} \times H^{2}(\Omega)$. In other words, we have shown that $H(u, u)$ is strictly convex on $V$. Taking into account that $\widetilde{L}(u)$ is linear, we have that $J(u)$ is strictly convex on $V$. Therefore, $J(u)$ has a unique minimizer, denoted by $w$, on $V$. In other words, $J^{\prime}(w)[v]=0$ for all $v \in V$, i.e.,

$$
H(w, v)=\widetilde{L}(v)
$$

for all $v \in V$. Now we need to show that (3.21) is valid for all $v \in\left(H^{1}(\Omega)\right)^{2} \times$ $H^{2}(\Omega)$, that is, $w$ indeed a weak solution. Given any $z=\left(z^{\prime}, z_{3}\right) \in H^{1}(\Omega) \times$ $H^{2}(\Omega)$, one can easily check that $\tilde{z}$ satisfies (3.9), where

$$
\begin{aligned}
& \widetilde{z}^{\prime}=z^{\prime}-\frac{1}{|\Omega|} \int_{\Omega} z^{\prime}-\left[\frac{1}{|\Omega|} \int_{\Omega} \frac{\left(\nabla z^{\prime}-\left(\nabla z^{\prime}\right)^{t}\right)}{2}\right]\left(x-x_{\Omega}\right)+\left(\theta-\theta_{\Omega}\right) \frac{1}{|\Omega|} \int_{\Omega} \nabla z_{3}, \\
& \tilde{z}_{3}=z_{3}-\frac{1}{|\Omega|} \int_{\Omega} z_{3}-\left(\frac{1}{|\Omega|} \int_{\Omega} \nabla z_{3}\right) \cdot\left(x-x_{\Omega}\right),
\end{aligned}
$$

and

$$
\theta_{\Omega}=\frac{1}{|\Omega|} \int_{\Omega} \theta, \quad x_{\Omega}=\frac{1}{|\Omega|} \int_{\Omega} x .
$$

Since $(\widehat{T}, \widehat{M})$ satisfies the compatibility condition (3.8), we conclude that

$$
H(w, z)=H(w, \widetilde{z})=\widetilde{L}(z)=\widetilde{L}(z) \quad \forall z \in\left(H^{1}(\Omega)\right)^{2} \times H^{2}(\Omega) .
$$

The estimate (3.18) is an easy consequence of (3.20) and the trace inequality.

\subsection{Global regularity}

To study the inverse problem, we also need a global regularity theorem for the shallow shell equations. To simplify our presentation, we impose a technical assumption on $\bar{\theta}$ (or $\theta$ ) in this section. Assume that $\bar{\theta}$ satisfies

$$
\bar{\theta}=\nabla \bar{\theta}=0 \quad \text { on } \quad \partial \Omega .
$$

We shall prove the following theorem.

Theorem 3.4. Assume that $\Omega$ is a bounded domain in $\mathbb{R}^{2}$ satisfying (3.1) whose boundary $\partial \Omega$ is of class $C^{4,1}$ with constants $A_{0}$ and $\rho_{0}$. Let $\lambda, \mu \in C^{1,1}(\bar{\Omega})$ satisfy (3.3) and $\bar{\theta} \in C^{2,1}(\bar{\Omega})$ satisfy (3.22) and

$$
\|\lambda\|_{C^{1,1}(\bar{\Omega})}+\|\mu\|_{C^{1,1}(\bar{\Omega})}+\|\bar{\theta}\|_{C^{2,1}(\bar{\Omega})} \leq A_{2} .
$$

Let $u \in\left(H^{1}(\Omega)\right)^{2} \times H^{2}(\Omega)$ be the weak solution of (3.4), (3.5) with Neumann boundary condition $(\widehat{T}, \widehat{M}) \in\left(H^{1 / 2}(\partial \Omega)\right)^{2} \times H^{3 / 2}(\partial \Omega)$ satisfying (3.8). Assume that $u$ satisfies the normalization conditions (3.9). Then there exists a constant $C>0$, depending on $A_{0}, A_{1}, A_{2}, \delta_{0}$ such that

$$
\left\|u^{\prime}\right\|_{H^{2}(\Omega)}+\|u\|_{H^{4}(\Omega)} \leq C\|(\widehat{T}, \widehat{M})\|_{\left(H^{1 / 2}(\partial \Omega)\right)^{2} \times H^{3 / 2}(\partial \Omega)} .
$$


Proof. To prove this theorem, it suffices to consider (3.4) with homogeneous Neumann boundary conditions. In view of (3.22), the boundary conditions (3.5) are simplified to

$$
\left\{\begin{array}{l}
n_{i j}\left(u^{\prime}\right) v_{j}=\rho_{0}^{-1} \widehat{T}_{i}, \\
m_{i j}\left(u_{3}\right) v_{i} v_{j}=\widehat{M}_{\nu}, \\
\partial_{i} m_{i j}\left(u_{3}\right) v_{j}+\partial_{s}\left(m_{i j}\left(u_{3}\right) v_{i} \tau_{j}\right)=-\partial_{s} \widehat{M}_{\tau},
\end{array}\right.
$$

where

$$
n_{i j}\left(u^{\prime}\right)=\frac{4 \lambda \mu}{\lambda+2 \mu} e_{k k}\left(u^{\prime}\right) \nu_{i}+4 \mu e_{i j}\left(u^{\prime}\right) \nu_{j}=\rho_{0}^{-1} \widehat{T}_{i} \quad \text { on } \quad \partial \Omega
$$

with $e_{i j}\left(u^{\prime}\right)=\frac{1}{2}\left(\partial_{i} u_{j}+\partial_{j} u_{i}\right)$. It is clear that boundary conditions (3.25) are decoupled. Using the result in [20, Proposition 8.1], one can find $\tilde{w}_{3}$ satisfying

$$
\left\{\begin{array}{l}
m_{i j}\left(\tilde{w}_{3}\right) v_{i} v_{j}=\widehat{M}_{\nu} \\
\partial_{i} m_{i j}\left(\tilde{w}_{3}\right) v_{j}+\partial_{s}\left(m_{i j}\left(\tilde{w}_{3}\right) v_{i} \tau_{j}\right)=-\partial_{s} \widehat{M}_{\tau}
\end{array}\right.
$$

on $\partial \Omega$ and the estimate

$$
\left\|\tilde{w}_{3}\right\|_{H^{4}(\Omega)} \leq C\|\widehat{M}\|_{H^{3 / 2}(\partial \Omega)} .
$$

Similarly, we can choose $\tilde{w}^{\prime}$ such that

$$
n_{i j}\left(\tilde{w}^{\prime}\right) v_{j}=\rho_{0}^{-1} \widehat{T}_{i} \quad \text { on } \quad \partial \Omega
$$

and

$$
\left\|\tilde{w}^{\prime}\right\|_{H^{2}(\Omega)} \leq C\|\widehat{T}\|_{\left(H^{1 / 2}(\partial \Omega)\right)^{2}} .
$$

The constant $C$ in (3.26) and (3.27) depend on $A_{0}, A_{1}, A_{2}, \delta_{0}$. By setting

$$
w^{\prime}=\tilde{w}^{\prime}-\frac{1}{|\Omega|} \int_{\Omega} \tilde{w}^{\prime}-\left[\frac{1}{|\Omega|} \int_{\Omega} \frac{\left(\nabla \tilde{w}^{\prime}-\left(\nabla \tilde{w}^{\prime}\right)^{t}\right)}{2}\right]\left(x-x_{\Omega}\right)+\left(\theta-\theta_{\Omega}\right) \frac{1}{|\Omega|} \int_{\Omega} \nabla \tilde{w}_{3}
$$

and

$$
w_{3}=\tilde{w}_{3}-\frac{1}{|\Omega|} \int_{\Omega} \tilde{w}_{3}-\left(\frac{1}{|\Omega|} \int_{\Omega} \nabla \tilde{w}_{3}\right) \cdot\left(x-x_{\Omega}\right),
$$

we can see that $\left(w^{\prime}, w_{3}\right)$ satisfies the boundary condition (3.25), the normalization conditions (3.9), and the estimate

$$
\left\|w^{\prime}\right\|_{H^{2}(\Omega)}+\left\|w_{3}\right\|_{H^{4}(\Omega)} \leq C\|(\widehat{T}, \widehat{M})\|_{\left(H^{1 / 2}(\partial \Omega)\right)^{2} \times H^{3 / 2}(\partial \Omega)},
$$

where $C$ depends on $A_{0}, A_{1}, A_{2}, \delta_{0}$. 
So now by letting $u=w+v$, we obtain that $v$ satisfies

$$
\left\{\begin{array}{l}
-\partial_{j} n_{i j}^{\theta}(v)=f_{i} \quad \text { in } \quad \Omega, \\
\partial_{i j}^{2} m_{i j}\left(v_{3}\right)-\partial_{j}\left(n_{i j}^{\theta}(v) \partial_{i} \theta\right)=f_{3} \quad \text { in } \Omega,
\end{array}\right.
$$

with homogeneous Neumann boundary conditions on $\partial \Omega$

$$
\left\{\begin{array}{l}
n_{i j}\left(v^{\prime}\right) v_{j}=0 \\
m_{i j}\left(v_{3}\right) v_{i} v_{j}=0 \\
\partial_{i} m_{i j}\left(v_{3}\right) v_{j}+\partial_{s}\left(m_{i j}\left(v_{3}\right) v_{i} \tau_{j}\right)=0
\end{array}\right.
$$

where $f=\left(f_{1}, f_{2}, f_{3}\right)=\left(f^{\prime}, f_{3}\right)$ is given by

$$
\left\{\begin{array}{l}
f_{i}=\partial_{j} n_{i j}^{\theta}(w), i=1,2 \\
f_{3}=-\partial_{i j}^{2} m_{i j}\left(w_{3}\right)+\partial_{j}\left(n_{i j}^{\theta}(w) \partial_{i} \theta\right)
\end{array}\right.
$$

Using the integration by parts, it is not hard to check that $f$ satisfies the following compatibility conditions

$$
\int_{\Omega} f=0, \int_{\Omega}\left(f_{1} x_{2}-f_{2} x_{1}\right)=0, \int_{\Omega}\left(f_{1} \theta+f_{3} x_{1}\right)=0, \int_{\Omega}\left(f_{2} \theta+f_{3} x_{2}\right)=0 .
$$

Now to obtain a global estimate for $v$, we decouple (3.29) as follows

$$
\left\{\begin{array}{l}
-\partial_{j} n_{i j}\left(v^{\prime}\right)=f_{i}+\frac{1}{2} \partial_{j}\left(\partial_{i} \theta \partial_{j} v_{3}+\partial_{j} \theta \partial_{i} v_{3}\right):=\tilde{f}_{i} \quad \text { in } \quad \Omega \\
\partial_{i j}^{2} m_{i j}\left(v_{3}\right)=f_{3}+\partial_{j}\left(n_{i j}^{\theta}(v) \partial_{i} \theta\right):=\tilde{f}_{3} \quad \text { in } \quad \Omega .
\end{array}\right.
$$

By (3.31) and straightforward computations, we can deduce that $\tilde{f}=\left(\tilde{f}_{1}, \tilde{f}_{2}, \tilde{f}_{3}\right)=$ $\left(\tilde{f}^{\prime}, \tilde{f}_{3}\right)$ satisfy

$$
\int_{\Omega} \tilde{f}=0, \int_{\Omega}\left(\tilde{f}_{1} x_{2}-\tilde{f}_{2} x_{1}\right)=0, \int_{\Omega} \tilde{f}_{3} x_{1}=\int_{\Omega} \tilde{f}_{3} x_{2}=0,
$$

which are the compatibility conditions for the existence of the boundary value problem (3.32) and (3.30). Recall the global estimate for the isotropic elasticity with homogeneous Neumann boundary condition, we have

$$
\begin{aligned}
\left\|v^{\prime}\right\|_{H^{2}(\Omega)} & \leq C\left(\rho_{0}^{2}\left\|\tilde{f}^{\prime}\right\|_{L^{2}(\Omega)}+\left\|v^{\prime}\right\|_{H^{1}(\Omega)}\right) \\
& \leq C\left(\rho_{0}^{2}\left\|f^{\prime}\right\|_{L^{2}(\Omega)}+\left\|v_{3}\right\|_{H^{2}(\Omega)}+\left\|v^{\prime}\right\|_{H^{1}(\Omega)}\right),
\end{aligned}
$$

where $C$ depend on $A_{0}, A_{1}, A_{2}, \delta_{0}$. For $v_{3}$, we use [20, Proposition 8.2] to obtain that

$$
\begin{aligned}
\left\|v_{3}\right\|_{H^{4}(\Omega)} & \leq C\left(\rho_{0}^{2}\left\|\tilde{f}_{3}\right\|_{L^{2}(\Omega)}+\left\|v_{3}\right\|_{H^{2}(\Omega)}\right) \\
& \leq C\left(\rho_{0}^{2}\left\|\tilde{f}_{3}\right\|_{L^{2}(\Omega)}+\left\|v_{3}\right\|_{H^{2}(\Omega)}+\left\|v^{\prime}\right\|_{H^{1}(\Omega)}\right) .
\end{aligned}
$$


The dependence of $C$ is the same as above. Putting (3.34) and (3.35) together yields

$$
\left\|v^{\prime}\right\|_{H^{2}(\Omega)}+\left\|v_{3}\right\|_{H^{4}(\Omega)} \leq C\left(\rho_{0}^{2}\|f\|_{L^{2}(\Omega)}+\left\|v_{3}\right\|_{H^{2}(\Omega)}+\left\|v^{\prime}\right\|_{H^{1}(\Omega)}\right) .
$$

Now using the weak formulation of the boundary value problem (3.32), (3.30), the Poincaré-Korn inequality (3.10), (3.28), we get from (3.36) that

$$
\begin{aligned}
\left\|v^{\prime}\right\|_{H^{2}(\Omega)}+\left\|v_{3}\right\|_{H^{4}(\Omega)} & \leq C\left(\rho_{0}^{2}\|f\|_{L^{2}(\Omega)}+\left\|v_{3}\right\|_{H^{2}(\Omega)}+\left\|v^{\prime}\right\|_{H^{1}(\Omega)}\right) \\
& \leq C \rho_{0}^{2}\|f\|_{L^{2}(\Omega)} \\
& \leq C\|(\widehat{T}, \widehat{M})\|_{\left(H^{1 / 2}(\partial \Omega)\right)^{2} \times H^{3 / 2}(\partial \Omega)} .
\end{aligned}
$$

Finally, combining (3.28) and (3.37) gives (3.24).

\section{Quantitative uniqueness estimates}

\subsection{Main theorems}

In this section, we would like to derive the three-ball inequalities for (1.1), which is a form of quantitative uniqueness estimate. The regularity of $\partial \Omega$ is irrelevant for the estimates derived here. But to make the paper consistent, we assume that $\Omega$ is at least a Lipschitz domain with constant $A_{0}$ and $\rho_{0}$. Let $\lambda(x), \mu(x)$ satisfy (3.3) and $\lambda, \mu, \bar{\theta}$ satisfy estimate (3.23). We now first state the main results of this section. Assume that $B_{\rho_{0} \bar{R}_{0}} \subset \Omega$ with $\bar{R}_{0} \leq 1$. Let us denote $U_{r}=\left(r u^{\prime}, u_{3}\right)=$ $\left(r u_{1}, r u_{2}, u_{3}\right)$. Then the following local estimates hold.

Theorem 4.1. There exists a positive number $R_{1}$, depending on $\delta_{0}, K_{1}, K_{2}$, such that if $0<r_{1}<r_{2}<r_{3} \leq \rho_{0} \bar{R}_{0}$ and $r_{1} / r_{3}<r_{2} / r_{3}<R_{1}$, then

$$
\int_{|x|<r_{2}}\left|U_{r_{2}}\right|^{2} d x \leq C_{1}\left(\int_{|x|<r_{1}}\left|U_{r_{1}}\right|^{2} d x\right)^{\tau}\left(\int_{|x|<r_{3}}\left|U_{r_{3}}\right|^{2} d x\right)^{1-\tau}
$$

for $\left(u^{\prime}, u_{3}\right) \in\left(H^{1}\left(B_{\rho_{0} \bar{R}_{0}}\right)\right)^{2} \times H^{3}\left(B_{\rho_{0} \bar{R}_{0}}\right)$ satisfying (1.1) in $B_{\rho_{0} \bar{R}_{0}}$, where $C_{1}>0$ and $0<\tau<1$ depend on $r_{1} / r_{3}, r_{2} / r_{3}, \delta_{0}, A_{2}$.

Remark 4.2. The estimate (4.1) is the three-ball inequality. Constants $C_{1}$ and $\tau$ appeared above can be explicitly written as $\tau=B /(E+B)$ and

$$
C_{1}=\max \left\{C_{0}\left[\left(\log \left(r_{1} / r_{3}\right)\right)^{2} /\left(\log \left(r_{2} / r_{3}\right)\right)^{2}\right]\left(r_{2} / r_{1}\right)^{2}, \exp \left(B \beta_{0}\right)\right\}\left(r_{3} / r_{1}\right)^{2 \tau},
$$

where $C_{0}>1$ and $\beta_{0}$ are constants depending on $\delta_{0}, A_{2}$ and

$$
\begin{aligned}
& E=E\left(r_{1} / r_{3}, r_{2} / r_{3}\right)=\left(\log \left(r_{1} / r_{3}\right)-1\right)^{2}-\left(\log \left(r_{2} / r_{3}\right)\right)^{2}, \\
& B=B\left(r_{2} / r_{3}\right)=-1-2 \log \left(r_{2} / r_{3}\right) .
\end{aligned}
$$


Remark 4.3. If $r_{3} \leq 1$, then (4.1) is reduced to

$$
\int_{|x|<r_{2}}|U|^{2} d x \leq \frac{C_{1}}{r_{2}^{2}}\left(\int_{|x|<r_{1}}|U|^{2} d x\right)^{\tau}\left(\int_{|x|<r_{3}}|U|^{2} d x\right)^{1-\tau} .
$$

By abuse of notation, we denote $U=\left(u^{\prime}, u_{3}\right)$.

Using the three-ball inequality, we can prove

Theorem 4.4. If $\left(u^{\prime}, u_{3}\right) \in\left(H^{1}\left(B_{\rho_{0}} \bar{R}_{0}\right)\right)^{2} \times H^{3}\left(B_{\rho_{0} \bar{R}_{0}}\right)$ is a nontrivial solution to (1.1), then we can find a constant $R_{2}$ depending on $\delta_{0}, A_{2}$ and a constant $m_{1}$ depending on $\delta_{0}, A_{2}$ and $\left\|U_{R_{2}^{2}}\right\|_{L^{2}\left(|x|<\rho_{0} R_{2}^{2}\right)} /\left\|U_{R_{2}^{4}}\right\|_{L^{2}\left(|x|<\rho_{0} R_{2}^{4}\right)}$ such that

$$
\int_{|x|<R}|U|^{2} d x \geq K R^{m_{1}},
$$

where $R$ is sufficiently small and the constant $K$ depends on $R_{2}$ and $U$.

In view of the standard unique continuation property for (1.1) in a connected domain containing the origin, if $u$ vanishes in a neighborhood of the origin then it vanishes identically in $\Omega$. Theorem 4.4 provides an upper bound on the vanishing order of a nontrivial solution to (1.1). The following doubling inequality is another quantitative estimate of the strong unique continuation for (1.1).

Theorem 4.5. Let $\left(u^{\prime}, u_{3}\right) \in\left(H^{1}\left(B_{\rho_{0} \bar{R}_{0}}\right)\right)^{2} \times H^{3}\left(B_{\rho_{0} \bar{R}_{0}}\right)$ be a nonzero solution to $(1.1)$. Then there exist positive constants $R_{3}$, depending on $\delta_{0}, A_{2},\left\|U_{R_{2}^{2}}\right\|_{L^{2}\left(|x|<\rho_{0} R_{2}^{2}\right)} /$ $\left\|U_{R_{2}^{4}}\right\|_{L^{2}\left(|x|<\rho_{0} R_{2}^{4}\right)}$, and $C_{2}$, depending on $\delta_{0}, A_{2}, m_{1}$, such that if $0<r \leq \rho_{0} R_{3}$, then

$$
\int_{|x| \leq 2 r}|U|^{2} d x \leq C_{2} \int_{|x| \leq r}|U|^{2} d x
$$

where $R_{2}$ and $m_{1}$ are the constants obtained in Theorem 4.4.

The rest of this section is devoted to the proofs of Theorem 4.1, 4.4, and 4.5.

\subsection{Preliminaries}

From now on, it suffices to take $\rho_{0}=1$. The first step is to transform the system (1.1) into a new system with uncoupled principal parts. To simplify the notation in the following proofs, we denote $u=u^{\prime}=\left(u_{1}, u_{2}\right)$ (suppress the prime), $w=$ $u_{3}$, and $v=\operatorname{div} u^{\prime}=\operatorname{div} u$. Putting (1.1) and the equation obtained by taking the divergence of the first system of (1.1) together, we come to the following new system

$$
\left\{\begin{aligned}
\Delta u & =P_{1}(D u, D v)+P_{2}\left(D^{2} w, D w\right) \\
\Delta v & =P_{3}(D u, D v)+P_{4}\left(D^{3} w, D^{2} w, D w\right) \\
\Delta^{2} w & =P_{5}\left(D^{3} w, D^{2} w, D w\right)+P_{6}(D u)
\end{aligned}\right.
$$


where $P_{1}-P_{6}$ are zeroth order operators with at least $L^{\infty}$ coefficients which are bounded by a constant depending on $\delta_{0}, A_{2}$.

To prove Theorem 4.1, the following interior estimate is useful. From now on, the notation $X \lesssim Y$ or $X \gtrsim Y$ means that $X \leq C Y$ or $X \geq C Y$ with some constant $C$ which could only depend on $\delta_{0}, A_{2}$.

Lemma 4.6. Let $(u, w) \in\left(H_{\mathrm{loc}}^{1}\left(B_{\bar{R}_{0}}\right)\right)^{2} \times H_{\mathrm{loc}}^{3}\left(B_{\bar{R}_{0}}\right)$ be a solution of $(1.1)$. Then for any $0<a_{3}<a_{1}<a_{2}<a_{4}$, there exists a constant $r_{0}$ with $a_{4} r_{0}<\bar{R}_{0}(<1)$ such that if $r \leq r_{0}$

$$
\begin{aligned}
& \sum_{|\alpha| \leq 2} \int_{a_{1} r<|x|<a_{2} r}|x|^{2|\alpha|}\left|D^{\alpha} u\right|^{2} d x+\sum_{|\alpha| \leq 4} \int_{a_{1} r<|x|<a_{2} r}|x|^{2|\alpha|}\left|D^{\alpha} w\right|^{2} d x \\
\leq & C_{3} \int_{a_{3} r<|x|<a_{4} r}\left(|u|^{2}+|w|^{2}\right) d x,
\end{aligned}
$$

where $C_{3}$ is independent of $r$ and $(u, w)$.

Proof. The proof here is motivated by the ideas used in [12, Corollary 17.1.4]. Let $X=B_{a_{4} r} \backslash \overline{B_{a_{3} r}}$ and $d(x)$ be the distant from $x \in X$ to $\mathbb{R}^{2} \backslash X$. We obtain from (1.1) that $u \in\left(H_{\mathrm{loc}}^{2}\left(B_{\bar{R}_{0}} \backslash\{0\}\right)\right)^{2}$ and $w \in H_{\mathrm{loc}}^{4}\left(B_{\bar{R}_{0}} \backslash\{0\}\right.$. Denote

$$
\mathcal{L}(x, D) u:=\frac{4 \lambda \mu}{\lambda+2 \mu} \nabla(\operatorname{div} u)+4 \mu \operatorname{div}(\operatorname{Sym}(\nabla u)) .
$$

Since $\mathcal{L}(x, D)$ and $\Delta^{2}$ are uniformly elliptic, it is obvious that

$$
\left\{\begin{aligned}
&\|f\|_{H^{2}\left(\mathbb{R}^{n}\right)} \lesssim\|\mathcal{L}(y, D) f\|_{L^{2}\left(\mathbb{R}^{n}\right)}+\|f\|_{L^{2}\left(\mathbb{R}^{n}\right)} \\
&\|g\|_{H^{4}\left(\mathbb{R}^{n}\right)} \lesssim\left\|\Delta^{2} g\right\|_{L^{2}\left(\mathbb{R}^{n}\right)}+\|g\|_{L^{2}\left(\mathbb{R}^{n}\right)}
\end{aligned}\right.
$$

for all $f \in H^{2}\left(\mathbb{R}^{n}\right), g \in H^{4}\left(\mathbb{R}^{n}\right)$ and any fixed $y$ in $\Omega$. Note that the absolute constant appearing in the first estimate of (4.7) can be chosen to be uniformly in $y \in \Omega$. By changing variables $x \rightarrow B^{-1} x$ in (4.7), we will have

$$
\left\{\begin{array}{l}
\sum_{|\alpha| \leq 1} B^{2-|\alpha|}\|f\|_{H^{2}\left(\mathbb{R}^{n}\right)} \lesssim\|\mathcal{L}(y, D) f\|_{L^{2}\left(\mathbb{R}^{n}\right)}+B^{2}\|f\|_{L^{2}\left(\mathbb{R}^{n}\right)} \\
\sum_{|\alpha| \leq 3} B^{4-|\alpha|}\left\|D^{\alpha} g\right\|_{L^{2}\left(\mathbb{R}^{n}\right)} \lesssim\left\|\Delta^{2} g\right\|_{L^{2}\left(\mathbb{R}^{n}\right)}+B^{4}\|g\|_{L^{2}\left(\mathbb{R}^{n}\right)}
\end{array}\right.
$$

for all $f \in H^{2}\left(\mathbb{R}^{n}\right)$ and $g \in H^{4}\left(\mathbb{R}^{n}\right)$. To apply (4.8) on $(u, w)$, we need to cut-off $(u, w)$. So let $\xi(x) \in C_{0}^{\infty}\left(\mathbb{R}^{n}\right)$ satisfy $0 \leq \xi(x) \leq 1$ and

$$
\xi(x)= \begin{cases}1, & |x|<1 / 4 \\ 0, & |x| \geq 1 / 2\end{cases}
$$


Let us denote $\xi_{y}(x)=\xi((x-y) / d(y))$. For $y \subset X$, we apply (4.8) to $\xi_{y}(x) u(x)$ and use the first equation of (1.1) to get that

$$
\begin{aligned}
& \sum_{|\alpha| \leq 2} B^{4-2|\alpha|} \int_{|x-y| \leq d(y) / 4}\left|D^{\alpha} u\right|^{2} d x \\
\lesssim & \sum_{|\alpha| \leq 1} \int_{|x-y| \leq d(y) / 2} d(y)^{-4+2|\alpha|}\left|D^{\alpha} u\right|^{2} d x+\int_{|x-y| \leq d(y) / 2}|\mathcal{L}(x, D) u|^{2} d x \\
& +\int_{|x-y| \leq d(y) / 2}|\mathcal{L}(x, D) u-\mathcal{L}(y, D) u|^{2} d x+B^{4} \int_{|x-y| \leq d(y) / 2}|u|^{2} d x \\
& +\sum_{|\alpha| \leq 2} \int_{|x-y| \leq d(y) / 2}\left|D^{\alpha} w\right|^{2} d x \\
\lesssim & \sum_{|\alpha| \leq 1} \int_{|x-y| \leq d(y) / 2} d(y)^{-4+2|\alpha|}\left|D^{\alpha} u\right|^{2} d x+r \sum_{|\alpha|=2} \int_{|x-y| \leq d(y) / 2}\left|D^{\alpha} u\right|^{2} d x \\
& +B^{4} \int_{|x-y| \leq d(y) / 2}|u|^{2} d x+\sum_{|\alpha| \leq 2} \int_{|x-y| \leq d(y) / 2}\left|D^{\alpha} w\right|^{2} d x .
\end{aligned}
$$

Now taking $B=M d(y)^{-1}$ for some positive constant $M$ and multiplying $d(y)^{4}$ on both sides of (4.9), we have

$$
\begin{aligned}
& \sum_{|\alpha| \leq 2} M^{4-2|\alpha|} \int_{|x-y| \leq d(y) / 4} d(y)^{2|\alpha|}\left|D^{\alpha} u\right|^{2} d x \\
\lesssim & \sum_{|\alpha| \leq 1} \int_{|x-y| \leq d(y) / 2} d(y)^{2|\alpha|}\left|D^{\alpha} u\right|^{2} d x+r \sum_{|\alpha|=2} \int_{|x-y| \leq d(y) / 2} d(y)^{4}\left|D^{\alpha} u\right|^{2} d x \\
& +M^{4} \int_{|x-y| \leq d(y) / 2}|u|^{2} d x+\sum_{|\alpha| \leq 2} \int_{|x-y| \leq d(y) / 2} d(y)^{4}\left|D^{\alpha} w\right|^{2} d x
\end{aligned}
$$

Integrating $d(y)^{-2} d y$ over $X$ on both sides of (4.10) and using Fubini's Theorem, we get that

$$
\begin{aligned}
& \sum_{|\alpha| \leq 2} M^{4-2|\alpha|} \int_{X} \int_{|x-y| \leq d(y) / 4} d(y)^{2|\alpha|-2}\left|D^{\alpha} u\right|^{2} d y d x \\
\lesssim & \sum_{|\alpha| \leq 1} \int_{X} \int_{|x-y| \leq d(y) / 2} d(y)^{2|\alpha|-2}\left|D^{\alpha} u\right|^{2} d y d x \\
& +M^{4} \int_{X} \int_{|x-y| \leq d(y) / 2} d(y)^{-2}|u|^{2} d y d x+r \sum_{|\alpha|=2} \int_{X} \int_{|x-y| \leq d(y) / 2} d(y)^{2}\left|D^{\alpha} u\right|^{2} d y d x \\
& +\sum_{|\alpha| \leq 2} \int_{X} \int_{|x-y| \leq d(y) / 2} d(y)^{2}\left|D^{\alpha} w\right|^{2} d y d x .
\end{aligned}
$$


Note that $|d(x)-d(y)| \leq|x-y|$. If $|x-y| \leq d(x) / 3$, then

$$
2 d(x) / 3 \leq d(y) \leq 4 d(x) / 3 .
$$

On the other hand, if $|x-y| \leq d(y) / 2$, then

$$
d(x) / 2 \leq d(y) \leq 3 d(x) / 2 .
$$

By (4.12) and (4.13), we have

$$
\left\{\begin{array}{l}
\int_{|x-y| \leq d(y) / 4} d(y)^{-2} d y \geq 9 / 16 \int_{|x-y| \leq d(x) / 6} d(x)^{-2} d y \geq 1 / 64 \int_{|y| \leq 1} d y, \\
\int_{|x-y| \leq d(y) / 2} d(y)^{-2} d y \leq 4 \int_{|x-y| \leq 3 d(x) / 4} d(x)^{-2} d y \leq 9 / 4 \int_{|y| \leq 1} d y
\end{array}\right.
$$

Combining (4.11)-(4.14), we obtain

$$
\begin{aligned}
& \sum_{|\alpha| \leq 2} M^{4-2|\alpha|} \int_{X} d(x)^{2|\alpha|}\left|D^{\alpha} u\right|^{2} d x \\
\lesssim & \sum_{|\alpha| \leq 1} \int_{X} d(x)^{2|\alpha|}\left|D^{\alpha} u\right|^{2} d x+r \sum_{|\alpha|=2} \int_{X} d(x)^{4}\left|D^{\alpha} u\right|^{2} d x \\
& +M^{4} \int_{X}|u|^{2} d x+\sum_{|\alpha| \leq 2} \int_{X} d(x)^{4}\left|D^{\alpha} w\right|^{2} d x .
\end{aligned}
$$

We can take $M$ large enough and $r$ small enough to absorb the first two terms on the right-hand side of (4.15). Thus we conclude that

$$
\begin{aligned}
& \sum_{|\alpha| \leq 2} M^{4-2|\alpha|} \int_{X} d(x)^{2|\alpha|}\left|D^{\alpha} u\right|^{2} d x \\
\lesssim & M^{4} \int_{X}|u|^{2} d x+\sum_{|\alpha| \leq 2} \int_{X} d(x)^{4}\left|D^{\alpha} w\right|^{2} d x .
\end{aligned}
$$

Similarly, we can apply (4.8) to $\xi_{y}(x) w(x)$ and use the second equation of (1.1) to get that

$$
\begin{aligned}
& \sum_{|\alpha| \leq 4} M^{8-2|\alpha|} \int_{X} d(x)^{2|\alpha|}\left|D^{\alpha} w\right|^{2} d x \\
\lesssim & M^{8} \int_{X}|w|^{2} d x+\sum_{|\alpha| \leq 1} \int_{X} d(x)^{8}\left|D^{\alpha} u\right|^{2} d x .
\end{aligned}
$$

Combining (4.16), (4.17) and letting $M$ be sufficiently large, we can eliminate the last terms of (4.16) and (4.17). After that we fix $M$ and obtain

$$
\begin{aligned}
& \sum_{|\alpha| \leq 2} \int_{X} d(x)^{2|\alpha|}\left|D^{\alpha} u\right|^{2} d x+\sum_{|\alpha| \leq 4} \int_{X} d(x)^{2|\alpha|}\left|D^{\alpha} w\right|^{2} d x \\
\lesssim & \int_{X}|u|^{2} d x+\int_{X}|w|^{2} d x .
\end{aligned}
$$


We recall that $X=B_{a_{4} r} \backslash \overline{B_{a_{3} r}}$ and note that $d(x) \geq \widehat{C} r$ if $x \in B_{a_{2} r} \backslash B_{a_{1} r}$, where $\widehat{C}$ is independent of $r$. Hence, (4.6) is an easy consequence of (4.18).

The next result follows from Lemma 4.6:

Corollary 4.7. Let $(u, w) \in\left(H_{\mathrm{loc}}^{1}\left(B_{\bar{R}_{0}}\right)\right)^{2} \times H_{\mathrm{loc}}^{3}\left(B_{\bar{R}_{0}}\right)$ be a solution of $(1.1)$ and $v=\operatorname{div} u$. Then for any $0<a_{3}<a_{1}<a_{2}<a_{4}$, there exists a constant $r_{0}$ satisfying $a_{4} r_{0}<\bar{R}_{0}$ such that if $r \leq r_{0}$, we have

$$
\begin{aligned}
& \sum_{|\alpha| \leq 1} \int_{a_{1} r<|x|<a_{2} r}|x|^{2|\alpha|+2}\left|D^{\alpha} v\right|^{2} d x \\
\leq & C_{3} \int_{a_{3} r<|x|<a_{4} r}\left(|u|^{2}+|w|^{2}\right) d x,
\end{aligned}
$$

where the constant $C_{3}$ is independent of $r$ and $(u, w)$.

\subsection{Proof of Theorem 4.1}

To begin, we recall a Carleman estimate with weight $\varphi_{\beta}=\varphi_{\beta}(x)=\exp \left(\frac{\beta}{2}(\log |x|)^{2}\right)$ given in [15].

Lemma 4.8. [15, Corollary 3.2] Given $\sigma_{1} \in \mathbb{Z}$ and $\sigma_{2} \in \mathbb{Z}$ there exist a sufficiently large number $\beta_{0}>0$ and a sufficiently small number $r_{0}>0$ depending on $n, l, \sigma_{1}$ and $\sigma_{2}$ such that for all $u \in U_{r_{0}}$ with $0<r_{0}<e^{-1}, \beta \geq \beta_{0}$, we have that

$$
\begin{aligned}
& \sum_{|\alpha| \leq 2 l} \beta^{3 l-2|\alpha|} \int \varphi_{\beta}^{2}|x|^{2 \sigma_{1}+2|\alpha|-n}(\log |x|)^{2 \sigma_{2}+2 l-2|\alpha|}\left|D^{\alpha} u\right|^{2} d x \\
\leq & \widetilde{C}_{0} \int \varphi_{\beta}^{2}|x|^{2 \sigma_{1}+4 l-n}(\log |x|)^{2 \sigma_{2}}\left|\Delta^{l} u\right|^{2} d x,
\end{aligned}
$$

where $U_{r_{0}}=\left\{u \in C_{0}^{\infty}\left(\mathbb{R}^{n} \backslash\{0\}\right): \operatorname{supp}(u) \subset B_{r_{0}}\right\}$ and $\widetilde{C}_{0}$ is a positive constant depending on $n$ and $l$. Here $e=\exp (1)$.

Remark 4.9. The estimate (4.20) in Lemma 4.8 remains valid if we assume $u \in$ $H_{\text {loc }}^{2 l}\left(\mathbb{R}^{n} \backslash\{0\}\right)$ with compact support. This can be easily seen by cutting off $u$ for small $|x|$ and regularizing.

We first consider the case where $0<r_{1}<r_{2}<R<1 / e$ and $B_{R} \subset \Omega$. The constant $R$ will be chosen later. To use the estimate (4.20), we need to cut-off $u$. So let $\xi(x) \in C_{0}^{\infty}\left(\mathbb{R}^{n}\right)$ satisfy $0 \leq \xi(x) \leq 1$ and

$$
\xi(x)= \begin{cases}0, & |x| \leq r_{1} / e \\ 1, & r_{1} / 2<|x|<e r_{2} \\ 0, & |x| \geq 3 r_{2}\end{cases}
$$


It is easy to check that for all multi-index $\alpha$

$$
\left\{\begin{array}{l}
\left|D^{\alpha} \xi\right|=O\left(r_{1}^{-|\alpha|}\right) \text { for all } r_{1} / e \leq|x| \leq r_{1} / 2 \\
\left|D^{\alpha} \xi\right|=O\left(r_{2}^{-|\alpha|}\right) \text { for all } e r_{2} \leq|x| \leq 3 r_{2}
\end{array}\right.
$$

Noting that the commutator $\left[\Delta^{l}, \xi\right]$ is a $2 l-1$ order differential operator and using the estimate (4.20) on $\xi u$ with parameters $\sigma_{1}=0, \sigma_{2}=0, l=1, n=2$, we can derive from the first equations of (4.5) and (4.21) that

$$
\begin{aligned}
& \sum_{|\alpha| \leq 1} \beta^{3-2|\alpha|} \int_{r_{1} / 2<|x|<e r_{2}} \varphi_{\beta}^{2}|x|^{2|\alpha|-2}(\log |x|)^{2-2|\alpha|}\left|D^{\alpha} u\right|^{2} d x \\
\lesssim & \sum_{|\alpha| \leq 2} \beta^{3-2|\alpha|} \int \varphi_{\beta}^{2}|x|^{2|\alpha|-2}(\log |x|)^{2-2|\alpha|}\left|D^{\alpha}(\xi u)\right|^{2} d x \\
\lesssim & \int \varphi_{\beta}^{2}|x|^{2}|\Delta(\xi u)|^{2} d x \\
\lesssim & \int \varphi_{\beta}^{2}|x|^{2}\left(|\Delta u|^{2}+\sum_{|\alpha| \leq 1}|[\Delta, \xi] u|^{2}\right) d x \\
\lesssim & \int_{r_{1} / 2<|x|<e r_{2}} \varphi_{\beta}^{2}|x|^{2}\left[\sum_{|\alpha| \leq 1}\left(\left|D^{\alpha} u\right|^{2}+\left|D^{\alpha} v\right|^{2}\right)+\sum_{|\alpha| \leq 2}\left|D^{\alpha} w\right|^{2}\right] d x \\
& +\int_{r_{1} / e<|x|<r_{1} / 2} \varphi_{\beta}^{2}|x|^{2}\left[\sum_{|\alpha| \leq 1}\left(|x|^{2|\alpha|-4}\left|D^{\alpha} u\right|^{2}+\left|D^{\alpha} v\right|^{2}\right)+\sum_{|\alpha| \leq 2}\left|D^{\alpha} w\right|^{2}\right] d x \\
& +\int_{e r_{2}<|x|<3 r_{2}} \varphi_{\beta}^{2}|x|^{2}\left[\sum_{|\alpha| \leq 1}\left(|x|^{2|\alpha|-4}\left|D^{\alpha} u\right|^{2}+\left|D^{\alpha} v\right|^{2}\right)+\sum_{|\alpha| \leq 2}\left|D^{\alpha} w\right|^{2}\right] d x .
\end{aligned}
$$

Similarity, applying (4.20) to $\xi v$ with parameters $\sigma_{1}=1, \sigma_{2}=0, l=1, n=2$, we can derive from the second equation of (4.5) and (4.21) that

$$
\begin{aligned}
& \sum_{|\alpha| \leq 1} \beta^{3-2|\alpha|} \int_{r_{1} / 2<|x|<e r_{2}} \varphi_{\beta}^{2}|x|^{2|\alpha|}(\log |x|)^{2-2|\alpha|}\left|D^{\alpha} v\right|^{2} d x \\
& \lesssim \int_{r_{1} / 2<|x|<e r_{2}} \varphi_{\beta}^{2}|x|^{4}\left[\sum_{|\alpha| \leq 1}\left(\left|D^{\alpha} u\right|^{2}+\left|D^{\alpha} v\right|^{2}\right)+\sum_{|\alpha| \leq 3}\left|D^{\alpha} w\right|^{2}\right] d x \\
& +\int_{r_{1} / e<|x|<r_{1} / 2} \varphi_{\beta}^{2}|x|^{4}\left[\sum_{|\alpha| \leq 1}\left(|x|^{2|\alpha|-4}\left|D^{\alpha} v\right|^{2}+\left|D^{\alpha} u\right|^{2}\right)+\sum_{|\alpha| \leq 3}\left|D^{\alpha} w\right|^{2}\right] d x \\
& \quad+\int_{e r_{2}<|x|<3 r_{2}} \varphi_{\beta}^{2}|x|^{4}\left[\sum_{|\alpha| \leq 1}\left(|x|^{2|\alpha|-4}\left|D^{\alpha} v\right|^{2}+\left|D^{\alpha} u\right|^{2}\right)+\sum_{|\alpha| \leq 3}\left|D^{\alpha} w\right|^{2}\right] d x .
\end{aligned}
$$


Finally, applying (4.20) to $\xi w$ with parameters $\sigma_{1}=0, \sigma_{2}=1, l=2, n=2$, we obtain from the third equation of (4.5) and (4.21) that

$$
\begin{aligned}
& \sum_{|\alpha| \leq 3} \beta^{6-2|\alpha|} \int_{r_{1} / 2<|x|<e r_{2}} \varphi_{\beta}^{2}|x|^{2|\alpha|-2}(\log |x|)^{6-2|\alpha|}\left|D^{\alpha} w\right|^{2} d x \\
\lesssim & \int_{r_{1} / 2<|x|<e r_{2}} \varphi_{\beta}^{2}|x|^{6}(\log |x|)^{2}\left[\sum_{|\alpha| \leq 1}\left|D^{\alpha} u\right|^{2}+\sum_{|\alpha| \leq 3}\left|D^{\alpha} w\right|^{2}\right] d x \\
& +\int_{r_{1} / e<|x|<r_{1} / 2} \varphi_{\beta}^{2}|x|^{6}(\log |x|)^{2}\left[\sum_{|\alpha| \leq 1}\left|D^{\alpha} u\right|^{2}+\sum_{|\alpha| \leq 3}|x|^{2|\alpha|-8}\left|D^{\alpha} w\right|^{2}\right] d x \\
& +\int_{e r_{2}<|x|<3 r_{2}} \varphi_{\beta}^{2}|x|^{6}(\log |x|)^{2}\left[\sum_{|\alpha| \leq 1}\left|D^{\alpha} u\right|^{2}+\sum_{|\alpha| \leq 3}|x|^{2|\alpha|-8}\left|D^{\alpha} w\right|^{2}\right] d x .
\end{aligned}
$$

Putting (4.22), (4.23), (4.24) together, we can take $\beta \geq \widetilde{\beta}_{0} \gg 1$ and $R \leq \widetilde{R}_{0} \ll 1$ such that the terms $\int_{r_{1} / 2<|x|<e r_{2}}(\cdots) d x$ on the right-hand side are absorbed by the corresponding terms on the left-hand side. In other words, for $\beta \geq \beta_{0}$ and $R \leq \widetilde{R}_{0}$, we have that

$$
\begin{aligned}
& \sum_{|\alpha| \leq 1} \beta^{3-2|\alpha|} \int_{r_{1} / 2<|x|<e r_{2}} \varphi_{\beta}^{2}|x|^{2|\alpha|-2}(\log |x|)^{2-2|\alpha|}\left|D^{\alpha} u\right|^{2} d x \\
& +\sum_{|\alpha| \leq 1} \beta^{3-2|\alpha|} \int_{r_{1} / 2<|x|<e r_{2}} \varphi_{\beta}^{2}|x|^{2|\alpha|}(\log |x|)^{2-2|\alpha|}\left|D^{\alpha} v\right|^{2} d x \\
& +\sum_{|\alpha| \leq 3} \beta^{6-2|\alpha|} \int_{r_{1} / 2<|x|<e r_{2}} \varphi_{\beta}^{2}|x|^{2|\alpha|-2}(\log |x|)^{6-2|\alpha|}\left|D^{\alpha} w\right|^{2} d x \\
& \lesssim \int_{r_{1} / e<|x|<r_{1} / 2} \varphi_{\beta}^{2}|x|^{-2}\left(\sum_{|\alpha| \leq 1}|x|^{2|\alpha|}\left|D^{\alpha} u\right|^{2}+|x|^{2|\alpha|+2}\left|D^{\alpha} v\right|^{2}\right) d x \\
& +\int_{r_{1} / e<|x|<r_{1} / 2} \varphi_{\beta}^{2}(\log |x|)^{2}|x|^{-2} \sum_{|\alpha| \leq 3}|x|^{2|\alpha|}\left|D^{\alpha} w\right|^{2} d x \\
& +\int_{e r_{2}<|x|<3 r_{2}} \varphi_{\beta}^{2}|x|^{-2}\left(\sum_{|\alpha| \leq 1}|x|^{2|\alpha|}\left|D^{\alpha} u\right|^{2}+|x|^{2|\alpha|+2}\left|D^{\alpha} v\right|^{2}\right) d x \\
& +\int_{e r_{2}<|x|<3 r_{2}} \varphi_{\beta}^{2}(\log |x|)^{2}|x|^{-2} \sum_{|\alpha| \leq 3}|x|^{2|\alpha|}\left|D^{\alpha} w\right|^{2} d x .
\end{aligned}
$$


Now using (4.6) and (4.19) in (4.25) leads to

$$
\begin{aligned}
& \left(\log r_{2}\right)^{2} r_{2}^{-2} \varphi_{\beta}^{2}\left(r_{2}\right) \int_{r_{1} / 2<|x|<r_{2}}|U|^{2} d x \\
\lesssim & \left(\log r_{1}\right)^{2} r_{1}^{-2} \varphi_{\beta}^{2}\left(r_{1} / e\right) \int_{r_{1} / 4<|x|<r_{1}}|U|^{2} d x \\
& +\left(\log r_{2}\right)^{2} r_{2}^{-2} \varphi_{\beta}^{2}\left(e r_{2}\right) \int_{2 r_{2}<|x|<4 r_{2}}|U|^{2} d x .
\end{aligned}
$$

Here $U=\left(u_{1}, u_{2}, w\right)=\left(u_{1}, u_{2}, u_{3}\right)$. Note that we have used the restriction $r_{1}<$ $r_{2}<1 / e$ in the above computations. Dividing by $\left(\log r_{2}\right)^{2} r_{2}^{-2} \varphi_{\beta}^{2}\left(r_{2}\right)$ on both sides of (4.26) implies

$$
\begin{aligned}
& \int_{r_{1} / 2<|x|<r_{2}}|U|^{2} d x \\
\lesssim & {\left[\left(\log r_{1}\right)^{2} /\left(\log r_{2}\right)^{2}\right]\left(r_{2} / r_{1}\right)^{2}\left[\varphi_{\beta}^{2}\left(r_{1} / e\right) / \varphi_{\beta}^{2}\left(r_{2}\right)\right] \int_{r_{1} / 4<|x|<r_{1}}|U|^{2} d x } \\
& +\left[\varphi_{\beta}^{2}\left(e r_{2}\right) / \varphi_{\beta}^{2}\left(r_{2}\right)\right] \int_{2 r_{2}<|x|<4 r_{2}}|U|^{2} d x \\
\lesssim & {\left[\left(\log r_{1}\right)^{2} /\left(\log r_{2}\right)^{2}\right]\left(r_{2} / r_{1}\right)^{2}\left[\varphi_{\beta}^{2}\left(r_{1} / e\right) / \varphi_{\beta}^{2}\left(r_{2}\right)\right] \int_{|x|<r_{1}}|U|^{2} d x } \\
& +\left[\left(\log r_{1}\right)^{2} /\left(\log r_{2}\right)^{2}\right]\left(r_{2} / r_{1}\right)^{2}\left[\varphi_{\beta}^{2}\left(e r_{2}\right) / \varphi_{\beta}^{2}\left(r_{2}\right)\right] \int_{|x|<1}|U|^{2} d x .
\end{aligned}
$$

Adding $\int_{|x|<r_{1} / 2}|U|^{2} d x$ to both sides of (4.27), we get for $\beta \geq \beta_{0}$ that

$$
\begin{aligned}
& \int_{|x|<r_{2}}|U|^{2} d x \\
\lesssim & {\left[\left(\log r_{1}\right)^{2} /\left(\log r_{2}\right)^{2}\right]\left(r_{2} / r_{1}\right)^{2}\left[\varphi_{\beta}^{2}\left(r_{1} / e\right) / \varphi_{\beta}^{2}\left(r_{2}\right)\right] \int_{|x|<r_{1}}|U|^{2} d x } \\
& +\left[\left(\log r_{1}\right)^{2} /\left(\log r_{2}\right)^{2}\right]\left(r_{2} / r_{1}\right)^{2}\left[\varphi_{\beta}^{2}\left(e r_{2}\right) / \varphi_{\beta}^{2}\left(r_{2}\right)\right] \int_{|x|<1}|U|^{2} d x .
\end{aligned}
$$

By denoting

$$
\begin{aligned}
& E=\beta^{-1} \log \left[\varphi_{\beta}^{2}\left(r_{1} / e\right) / \varphi_{\beta}^{2}\left(r_{2}\right)\right]=\left(\log r_{1}-1\right)^{2}-\left(\log r_{2}\right)^{2}>0, \\
& B=-\beta^{-1} \log \left[\varphi_{\beta}^{2}\left(e r_{2}\right) / \varphi_{\beta}^{2}\left(r_{2}\right)\right]=-1-2 \log r_{2}>0,
\end{aligned}
$$


(4.28) becomes

$$
\begin{aligned}
& \int_{|x|<r_{2}}|U|^{2} d x \\
\lesssim & {\left[\left(\log r_{1}\right)^{2} /\left(\log r_{2}\right)^{2}\right]\left(r_{2} / r_{1}\right)^{2} \times } \\
& \left(\exp (E \beta) \int_{|x|<r_{1}}\left|U_{r_{1}}\right|^{2} d x+\exp (-B \beta) \int_{|x|<1}|U|^{2} d x\right) .
\end{aligned}
$$

To further simplify the terms on the right-hand side of (4.29), we consider two cases. If

$$
\int_{|x|<r_{1}}|U|^{2} d x \neq 0
$$

and

$$
\exp \left(E \beta_{0}\right) \int_{|x|<r_{1}}|U|^{2} d x<\exp \left(-B \beta_{0}\right) \int_{|x|<1}|U|^{2} d x
$$

then we can pick a $\beta>\beta_{0}$ such that

$$
\exp (E \beta) \int_{|x|<r_{1}}|U|^{2} d x=\exp (-B \beta) \int_{|x|<1}|U|^{2} d x .
$$

Using such $\beta$, we obtain from (4.29) that

$$
\begin{aligned}
& \int_{|x|<r_{2}}|U|^{2} d x \\
\lesssim & {\left[\left(\log r_{1}\right)^{2} /\left(\log r_{2}\right)^{2}\right]\left(r_{2} / r_{1}\right)^{2} \exp (E \beta) \int_{|x|<r_{1}}|U|^{2} d x } \\
\lesssim & {\left[\left(\log r_{1}\right)^{2} /\left(\log r_{2}\right)^{2}\right]\left(r_{2} / r_{1}\right)^{2}\left(\int_{|x|<r_{1}}|U|^{2} d x\right)^{\frac{B}{E+B}}\left(\int_{|x|<1}|U|^{2} d x\right)^{\frac{E}{E+B}} . }
\end{aligned}
$$

If

$$
\int_{|x|<r_{1}}|U|^{2} d x=0
$$

then it follows from (4.29) that

$$
\int_{|x|<r_{2}}|U|^{2} d x=0
$$

since we can take $\beta$ arbitrarily large. The three-sphere inequality obviously holds.

On the other hand, if

$$
\exp \left(-B \beta_{0}\right) \int_{|x|<1}|U|^{2} d x \leq \exp \left(E \beta_{0}\right) \int_{|x|<r_{1}}|U|^{2} d x
$$


then we have

$$
\begin{aligned}
& \int_{|x|<r_{2}}|U|^{2} d x \\
\leq & \left(\int_{|x|<1}|U|^{2} d x\right)^{\frac{B}{E+B}}\left(\int_{|x|<1}|U|^{2} d x\right)^{\frac{E}{E+B}} \\
\leq & \exp \left(B \beta_{0}\right)\left(\int_{|x|<r_{1}}|U|^{2} d x\right)^{\frac{B}{E+B}}\left(\int_{|x|<1}|U|^{2} d x\right)^{\frac{E}{E+B}} .
\end{aligned}
$$

Putting together (4.30), (4.31), we arrive at

$$
\int_{|x|<r_{2}}|U|^{2} d x \leq \tilde{C}_{3}\left(\int_{|x|<r_{1}}|U|^{2} d x\right)^{\frac{B}{E+B}}\left(\int_{|x|<1}|U|^{2} d x\right)^{\frac{E}{E+B}},
$$

where $\tilde{C}_{3}=\max \left\{\tilde{C}_{2}\left[\left(\log r_{1}\right)^{2} /\left(\log r_{2}\right)^{2}\right]\left(r_{2} / r_{1}\right)^{2}, \exp \left(B \beta_{0}\right)\right\}$ for some positive constant $\tilde{C}_{2}$, depending on $\delta_{0}, A_{2}$.

Now for the general case, we take $R_{1}=\widetilde{R}_{0}$ and consider $0<r_{1}<r_{2}<r_{3} \leq$ $\rho_{0} \bar{R}_{0}<1$ with $r_{1} / r_{3}<r_{2} / r_{3} \leq R_{1}$. By defining $\widehat{u}(y):=r_{3} u\left(r_{3} y\right), \widehat{w}(y):=$ $w\left(r_{3} y\right), \widehat{\lambda}(y):=\lambda\left(r_{3} y\right), \widehat{\mu}(y):=\mu\left(r_{3} y\right), \widehat{\theta}(y)=\theta\left(r_{3} y\right)$, we can see that the system (1.1) is invariant under this scaling. On the other hand, $\widehat{\lambda}(y), \widehat{\mu}(y)$ and $\widehat{\theta}(y)$ satisfy (3.23), respectively, with the same constants. Therefore, from (4.32), we get that

$$
\int_{|y|<r_{2} / r_{3}}|\widehat{U}|^{2} d y \leq \tilde{C}_{1}\left(\int_{|y|<r_{1} / r_{3}}|\widehat{U}|^{2} d y\right)^{\tau}\left(\int_{|y|<1}|\widehat{U}|^{2} d y\right)^{1-\tau}
$$

where $|\widehat{U}|^{2}=|\widehat{u}|^{2}+|\widehat{w}|^{2}, \tau=B /(E+B)$ with

$$
\begin{aligned}
& E=E\left(r_{1} / r_{3}, r_{2} / r_{3}\right)=\left(\log \left(r_{1} / r_{3}\right)-1\right)^{2}-\left(\log \left(r_{2} / r_{3}\right)\right)^{2}, \\
& B=B\left(r_{2} / r_{3}\right)=-1-2 \log \left(r_{2} / r_{3}\right),
\end{aligned}
$$

and $\tilde{C}_{1}=\max \left\{\tilde{C}_{2}\left[\left(\log r_{1} / r_{3}\right)^{2} /\left(\log r_{2} / r_{3}\right)^{2}\right]\left(r_{2} / r_{1}\right)^{2}, \exp \left(B \beta_{0}\right)\right\}$. Rewriting (4.33) with the original variables yields

$$
\int_{|x|<r_{2}}\left|U_{r_{2}}\right|^{2} d x \leq C_{1}\left(\int_{|x|<r_{1}}\left|U_{r_{1}}\right|^{2} d x\right)^{\tau}\left(\int_{|x|<r_{3}}\left|U_{r_{3}}\right|^{2} d x\right)^{1-\tau}
$$

with $C_{1}=\tilde{C}_{1}\left(r_{3} / r_{1}\right)^{2 \tau}$.

\subsection{Proof of Theorems 4.4 and 4.5}

In this section we prove Theorem 4.4 and 4.5. We begin with another Carleman estimate derived in [15, Lemma 2.1]: for any $f \in C_{0}^{\infty}\left(\mathbb{R}^{n} \backslash\{0\}\right)$ and for any $m \in$ 
$\left\{j+\frac{1}{2}, j \in \mathbb{N}\right\}$ we have

$$
\sum_{|\alpha| \leq 2 l} \int m^{2 l-2|\alpha|}|x|^{-2 m+2|\alpha|-n}\left|D^{\alpha} f\right|^{2} d x \leq C \int|x|^{-2 m+4 l-n}\left|\Delta^{l} f\right|^{2} d x,
$$

where $C$ depends only on the dimension $n$ and the power $l$.

Remark 4.10. Using the cut-off function and regularization, estimate (4.34) remains valid for any fixed $m$ if $f \in H_{\mathrm{loc}}^{2 l}\left(\mathbb{R}^{n} \backslash\{0\}\right)$ with compact support.

In view of Remark 4.10, we define $\chi(x) \in C_{0}^{\infty}\left(\mathbb{R}^{2} \backslash\{0\}\right)$ such that

$$
\chi(x)=\left\{\begin{array}{lll}
0 & \text { if } \quad|x| \leq \delta / 3 \\
1 & \text { in } \quad \delta / 2 \leq|x| \leq\left(R_{0}+1\right) R_{0} R / 4=r_{4} R \\
0 & \text { if } \quad 2 r_{4} R \leq|x|
\end{array}\right.
$$

where $\delta \leq R_{0}^{2} R / 4, R_{0}>0$ is a small number which will be chosen later and $R$ is given by $R=(\gamma m)^{-1}$, where $\gamma>0$ is a large constant which will be chosen later. In view of the definition of $\chi$, it is easy to see that for all multi-index $\alpha$

$$
\left\{\begin{array}{l}
\left|D^{\alpha} \chi\right|=O\left(\delta^{-|\alpha|}\right) \text { for all } \delta / 3<|x|<\delta / 2 \\
\left|D^{\alpha} \chi\right|=O\left(\left(r_{4} R\right)^{-|\alpha|}\right) \text { for all } r_{4} R<|x|<2 r_{4} R .
\end{array}\right.
$$

Using the estimate (4.34) to $\chi u$ with parameters $l=1, n=2$ and the equations (4.5), (4.35), the same arguments as (4.22) arrive that

$$
\begin{aligned}
& \sum_{|\alpha| \leq 2} m^{2-2|\alpha|} \int_{\delta / 2 \leq|x| \leq r_{4} R}|x|^{-2 m+2|\alpha|-2}\left|D^{\alpha} u\right|^{2} d x \\
\lesssim & \int_{\delta / 2 \leq|x| \leq r_{4} R}|x|^{-2 m+2}\left[\sum_{|\alpha| \leq 1}\left(\left|D^{\alpha} u\right|^{2}+\left|D^{\alpha} v\right|^{2}\right)+\sum_{|\alpha| \leq 2}\left|D^{\alpha} w\right|^{2}\right] d x \\
& +\int_{\delta / 3<|x|<\delta / 2}|x|^{-2 m+2}\left[\sum_{|\alpha| \leq 1}\left(|x|^{2|\alpha|-4}\left|D^{\alpha} u\right|^{2}+\left|D^{\alpha} v\right|^{2}\right)+\sum_{|\alpha| \leq 2}\left|D^{\alpha} w\right|^{2}\right] d x \\
& +\int_{r_{4} R<|x|<2 r_{4} R}|x|^{-2 m+2}\left[\sum_{|\alpha| \leq 1}\left(|x|^{2|\alpha|-4}\left|D^{\alpha} u\right|^{2}+\left|D^{\alpha} v\right|^{2}\right)+\sum_{|\alpha| \leq 2}\left|D^{\alpha} w\right|^{2}\right] d x .
\end{aligned}
$$


Similarity, applying (4.34) to $\chi v$ with parameters $m=m-1, l=1, n=2$, we can derive from (4.5) and (4.35) that

$$
\begin{aligned}
& \sum_{|\alpha| \leq 2}(m-1)^{2-2|\alpha|} \int_{\delta / 2 \leq|x| \leq r_{4} R}|x|^{-2 m+2|\alpha|}\left|D^{\alpha} v\right|^{2} d x \\
& \lesssim \int_{\delta / 2 \leq|x| \leq r_{4} R}|x|^{-2 m+4}\left[\sum_{|\alpha| \leq 1}\left(\left|D^{\alpha} u\right|^{2}+\left|D^{\alpha} v\right|^{2}\right)+\sum_{|\alpha| \leq 3}\left|D^{\alpha} w\right|^{2}\right] d x \\
& +\int_{\delta / 3<|x|<\delta / 2}|x|^{-2 m+4}\left[\sum_{|\alpha| \leq 1}\left(|x|^{2|\alpha|-4}\left|D^{\alpha} v\right|^{2}+\left|D^{\alpha} u\right|^{2}\right)+\sum_{|\alpha| \leq 3}\left|D^{\alpha} w\right|^{2}\right] d x \\
& +\int_{r_{4} R<|x|<2 r_{4} R}|x|^{-2 m+4}\left[\sum_{|\alpha| \leq 1}\left(|x|^{2|\alpha|-4}\left|D^{\alpha} v\right|^{2}+\left|D^{\alpha} u\right|^{2}\right)+\sum_{|\alpha| \leq 3}\left|D^{\alpha} w\right|^{2}\right] d x .
\end{aligned}
$$

Next applying (4.34) to $\chi w$ with parameters $l=2, n=2$, we get from (4.5) and (4.35) that

$$
\begin{aligned}
& \sum_{|\alpha| \leq 3} m^{4-2|\alpha|} \int_{\delta / 2 \leq|x| \leq r_{4} R}|x|^{-2 m+2|\alpha|-2}\left|D^{\alpha} w\right|^{2} d x \\
& \lesssim \int_{\delta / 2 \leq|x| \leq r_{4} R}|x|^{-2 m+6}\left[\sum_{|\alpha| \leq 1}\left|D^{\alpha} u\right|^{2}+\sum_{|\alpha| \leq 3}\left|D^{\alpha} w\right|^{2}\right] d x \\
& +\int_{\delta / 3<|x|<\delta / 2}|x|^{-2 m+6}\left[\sum_{|\alpha| \leq 1}\left|D^{\alpha} u\right|^{2}+\sum_{|\alpha| \leq 3}|x|^{2|\alpha|-8}\left|D^{\alpha} w\right|^{2}\right] d x \\
& +\int_{r_{4} R<|x|<2 r_{4} R}|x|^{-2 m+6}\left[\sum_{|\alpha| \leq 1}\left|D^{\alpha} u\right|^{2}+\sum_{|\alpha| \leq 3}|x|^{2|\alpha|-8}\left|D^{\alpha} w\right|^{2}\right] d x .
\end{aligned}
$$


Adding (4.36), $K_{1} \times(4.37)$ and $K_{2} m^{2} \times(4.38)$ together, we obtain that

$$
\begin{aligned}
& \sum_{|\alpha| \leq 2} m^{2-2|\alpha|} \int_{\delta / 2 \leq|x| \leq r_{4} R}|x|^{-2 m+2|\alpha|-2}\left|D^{\alpha} u\right|^{2} d x \\
& +K_{1} \sum_{|\alpha| \leq 2}(m-1)^{2-2|\alpha|} \int_{\delta / 2 \leq|x| \leq r_{4} R}|x|^{-2 m+2|\alpha|}\left|D^{\alpha} v\right|^{2} d x \\
& +K_{2} \sum_{|\alpha| \leq 3} m^{4-2|\alpha|} \int_{\delta / 2 \leq|x| \leq r_{4} R}|x|^{-2 m+2|\alpha|-2}\left|D^{\alpha} w\right|^{2} d x \\
& \lesssim \int_{\delta / 2 \leq|x| \leq r_{4} R}|x|^{-2 m+2}\left[\sum_{|\alpha| \leq 1}\left(\left|D^{\alpha} u\right|^{2}+\left|D^{\alpha} v\right|^{2}\right)+\sum_{|\alpha| \leq 2}\left|D^{\alpha} w\right|^{2}\right] d x \\
& +K_{1} \int_{\delta / 2 \leq|x| \leq r_{4} R}|x|^{-2 m+4}\left[\sum_{|\alpha| \leq 1}\left(\left|D^{\alpha} u\right|^{2}+\left|D^{\alpha} v\right|^{2}\right)+\sum_{|\alpha| \leq 3}\left|D^{\alpha} w\right|^{2}\right] d x \\
& +K_{2} \int_{\delta / 2 \leq|x| \leq r_{4} R}|x|^{-2 m+6}\left[\sum_{|\alpha| \leq 1}\left|D^{\alpha} u\right|^{2}+\sum_{|\alpha| \leq 3}\left|D^{\alpha} w\right|^{2}\right] d x \\
& +\int_{\delta / 3<|x|<\delta / 2}(\cdots)+\int_{r_{4} R<|x|<2 r_{4} R}(\cdots) .
\end{aligned}
$$

Now we choose $K_{1}$ sufficiently large such that the terms

$$
\int_{\delta / 2 \leq|x| \leq r_{4} R}|x|^{-2 m+2} \sum_{|\alpha|=1}\left|D^{\alpha} v\right|^{2} d x
$$

on the right-hand side of (4.39) are absorbed by its left-hand side. After that the constant $K_{1}$ is fixed. We continue to choose $K_{2}$ large enough such that

$$
K_{1} \int_{\delta / 2 \leq|x| \leq r_{4} R}|x|^{-2 m+4} \sum_{|\alpha|=3}\left|D^{\alpha} w\right|^{2} d x
$$

on the right-hand side of (4.39) are absorbed by its left-hand side. Then we fix $K_{2}$. To eliminate other terms inside the integral $\int_{\delta / 2 \leq|x| \leq r_{4} R}$ on the right-hand side of (4.39), we recall that $R=(\gamma m)^{-1}$. So by choosing $\gamma \geq \gamma_{0}$ and $m \geq m_{0}^{\prime}$ with large 
$\gamma_{0}$ and $m_{0}^{\prime}$, we have that

$$
\begin{aligned}
& \sum_{|\alpha| \leq 2} m^{2-2|\alpha|} \int_{\delta / 2 \leq|x| \leq r_{4} R}|x|^{-2 m+2|\alpha|-2}\left|D^{\alpha} u\right|^{2} d x \\
& +\sum_{|\alpha| \leq 2}(m-1)^{2-2|\alpha|} \int_{\delta / 2 \leq|x| \leq r_{4} R}|x|^{-2 m+2|\alpha|}\left|D^{\alpha} v\right|^{2} d x \\
& +\sum_{|\alpha| \leq 3} m^{6-2|\alpha|} \int_{\delta / 2 \leq|x| \leq r_{4} R}|x|^{-2 m+2|\alpha|-2}\left|D^{\alpha} w\right|^{2} d x \\
& \lesssim \int_{\delta / 3<|x|<\delta / 2}|x|^{-2 m-2}\left(\sum_{|\alpha| \leq 1}|x|^{2|\alpha|}\left|D^{\alpha} u\right|^{2}+|x|^{2|\alpha|+2}\left|D^{\alpha} v\right|^{2}\right) d x \\
& +\int_{\delta / 3<|x|<\delta / 2}|x|^{-2 m-2} m^{2} \sum_{|\alpha| \leq 3}|x|^{2|\alpha|}\left|D^{\alpha} w\right|^{2} d x \\
& +\int_{r_{4} R<|x|<2 r_{4} R}|x|^{-2 m-2}\left(\sum_{|\alpha| \leq 1}|x|^{2|\alpha|}\left|D^{\alpha} u\right|^{2}+|x|^{2|\alpha|+2}\left|D^{\alpha} v\right|^{2}\right) d x \\
& +\int_{r_{4} R<|x|<2 r_{4} R}|x|^{-2 m-2} m^{2} \sum_{|\alpha| \leq 3}|x|^{2|\alpha|}\left|D^{\alpha} w\right|^{2} d x
\end{aligned}
$$

Note that $R_{0}^{2} \leq r_{4}$ provided $R_{0} \leq 1 / 3$. Also, if $R_{0} \leq 1 / 3$, it is obvious that $2 r_{4} \leq R_{0}$. Dividing $m^{2}$ on both sides of (4.40) and using (4.19) and (4.6) in (4.40), it obtains that

$$
\begin{aligned}
& (2 \delta)^{-2 m-2} \int_{\delta / 2<|x| \leq 2 \delta}|U|^{2} d x+\left(R_{0}^{2} R\right)^{-2 m-2} \int_{2 \delta<|x| \leq R_{0}^{2} R}|U|^{2} d x \\
\lesssim & \int_{\delta / 2 \leq|x| \leq r_{4} R}|x|^{-2 m-2}|U|^{2} d x \\
\leq & C^{\prime}(\delta / 3)^{-2 m-2} \int_{|x| \leq \delta}|U|^{2} d x+C^{\prime \prime}\left(r_{4} R\right)^{-2 m-2} \int_{|x| \leq R_{0} R}|U|^{2} d x,
\end{aligned}
$$

where $C^{\prime}$ and $C^{\prime \prime}$ absolute constants. From now on, we need to trace the constants to make the estimates more clearly. Adding $(2 \delta)^{-2 m-2} \int_{|x| \leq \delta / 2}|U|^{2} d x$ to both sides 
of (4.41), we have that

$$
\begin{aligned}
& \frac{1}{2}(2 \delta)^{-2 m-2} \int_{|x| \leq 2 \delta}|U|^{2} d x+\left(R_{0}^{2} R\right)^{-2 m-2} \int_{|x| \leq R_{0}^{2} R}|U|^{2} d x \\
= & \frac{1}{2}(2 \delta)^{-2 m-2} \int_{|x| \leq 2 \delta}|U|^{2} d x+\left(R_{0}^{2} R\right)^{-2 m-2} \int_{|x| \leq 2 \delta}|U|^{2} d x \\
& +\left(R_{0}^{2} R\right)^{-2 m-2} \int_{2 \delta<|x| \leq R_{0}^{2} R}|U|^{2} d x \\
\leq & \frac{1}{2}(2 \delta)^{-2 m-2} \int_{|x| \leq 2 \delta}|U|^{2} d x+\frac{1}{2}(2 \delta)^{-2 m-2} \int_{|x| \leq 2 \delta}|U|^{2} d x \\
& +\left(R_{0}^{2} R\right)^{-2 m-2} \int_{2 \delta<|x| \leq R_{0}^{2} R}|U|^{2} d x \\
\leq & \left(C^{\prime}+1\right)(\delta / 3)^{-2 m-2} \int_{|x| \leq \delta}|U|^{2} d x+C^{\prime \prime}\left(r_{4} R\right)^{-2 m-2} \int_{|x| \leq R_{0} R}|U|^{2} d x \\
= & \left(C^{\prime}+1\right)(\delta / 3)^{-2 m-2} \int_{|x| \leq \delta}|U|^{2} d x \\
& +\left(R_{0}^{2} R\right)^{-2 m-2} C^{\prime \prime}\left(\frac{R_{0}^{2}}{r_{4}}\right)^{2 m+2} \int_{|x| \leq R_{0} R}|U|^{2} d x .
\end{aligned}
$$

We now observe that

$$
\begin{aligned}
& C^{\prime \prime}\left(\frac{R_{0}^{2}}{r_{4}}\right)^{2 m+2}=C^{\prime \prime}\left(\frac{4 R_{0}}{R_{0}+1}\right)^{2 m+2} \\
\leq & C^{\prime \prime}\left(4 R_{0}\right)^{2 m+2} \leq \exp (-2 m)
\end{aligned}
$$

for all $R_{0}<e^{-1} / 4$ and $m \geq m_{0}$, where $m_{0}$ depends on $C^{\prime \prime}$ and $R_{0}$. Thus, we obtain that

$$
\begin{aligned}
& \frac{1}{2}(2 \delta)^{-2 m-2} \int_{|x| \leq 2 \delta}|U|^{2} d x+\left(R_{0}^{2} R\right)^{-2 m-2} \int_{|x| \leq R_{0}^{2} R}|U|^{2} d x \\
\leq & \left(C^{\prime}+1\right)(\delta / 3)^{-2 m-2} \int_{|x| \leq \delta}|U|^{2} d x \\
& +\left(R_{0}^{2} R\right)^{-2 m-2} \exp (-2 m) \int_{|x| \leq R_{0} R}|U|^{2} d x .
\end{aligned}
$$

It should be noted that (4.43) is valid for all $m=j+\frac{1}{2}$ with $j \in \mathbb{N}$ and $j \geq j_{0}$, where $j_{0}$ depends on $R_{0}$. Setting $R_{j}=\left(\gamma\left(j+\frac{1}{2}\right)\right)^{-1}$ and using the relation $m=$ 
$(\gamma R)^{-1}$, we get from (4.43) that

$$
\begin{aligned}
& \frac{1}{2}(2 \delta)^{-2 m-2} \int_{|x| \leq 2 \delta}|U|^{2} d x+\left(R_{0}^{2} R_{j}\right)^{-2 m-2} \int_{|x| \leq R_{0}^{2} R_{j}}|U|^{2} d x \\
\leq & \left(C^{\prime}+1\right)(\delta / 3)^{-2 m-2} \int_{|x| \leq \delta}|U|^{2} d x \\
& +\left(R_{0}^{2} R_{j}\right)^{-2 m-2} \exp \left(-2 c R_{j}^{-1}\right) \int_{|x| \leq R_{0} R_{j}}|U|^{2} d x
\end{aligned}
$$

for all $j \geq j_{0}$ and $c=\gamma^{-1}$. We now observe that

$$
R_{j+1}<R_{j}<2 R_{j+1} \text { for all } j \in \mathbb{N} .
$$

Thus, if $R_{j+1}<r \leq R_{j}$, we can conclude that

$$
\left\{\begin{array}{l}
\int_{|x| \leq R_{0}^{2} r}|U|^{2} d x \leq \int_{|x| \leq R_{0}^{2} R_{j}}|U|^{2} d x \\
\exp \left(-2 c R_{j}^{-1}\right) \int_{|x| \leq R_{0} R_{j}}|U|^{2} d x \leq \exp \left(-c r^{-1}\right) \int_{|x| \leq r}|U|^{2} d x
\end{array}\right.
$$

where we have used the inequality $R_{0} R_{j}<2 R_{0} R_{j+1} \leq R_{j+1} /(2 e)<R_{j+1}$ to derive the second inequality above. Namely, we have from (4.44) and (4.45) that

$$
\begin{aligned}
& \frac{1}{2}(2 \delta)^{-2 m-2} \int_{|x| \leq 2 \delta}|U|^{2} d x+\left(R_{0}^{2} R_{j}\right)^{-2 m-2} \int_{|x| \leq R_{0}^{2} r}|U|^{2} d x \\
\leq & \left(C^{\prime}+1\right)(\delta / 3)^{-2 m-2} \int_{|x| \leq \delta}|U|^{2} d x \\
& +\left(R_{0}^{2} R_{j}\right)^{-2 m-2} \exp \left(-c r^{-1}\right) \int_{|x| \leq r}|U|^{2} d x .
\end{aligned}
$$

If there exists $s \in \mathbb{N}$ such that

$$
R_{j+1}<R_{0}^{2 s} \leq R_{j} \quad \text { for some } \quad j \geq j_{0},
$$

then replacing $r$ by $R_{0}^{2 s}$ in (4.46) leads to

$$
\begin{aligned}
& \frac{1}{2}(2 \delta)^{-2 m-2} \int_{|x| \leq 2 \delta}|U|^{2} d x+\left(R_{0}^{2} R_{j}\right)^{-2 m-2} \int_{|x| \leq R_{0}^{2 s+2}}|U|^{2} d x \\
\leq & \left(C^{\prime}+1\right)(\delta / 3)^{-2 m-2} \int_{|x| \leq \delta}|U|^{2} d x \\
& +\left(R_{0}^{2} R_{j}\right)^{-2 m-2} \exp \left(-c R_{0}^{-2 s}\right) \int_{|x| \leq R_{0}^{2 s}}|U|^{2} d x .
\end{aligned}
$$


Here $s$ and $R_{0}$ are yet to be determined. The trick now is to find suitable $s$ and $R_{0}$ satisfying (4.47) and the inequality

$$
\exp \left(-c R_{0}^{-2 s}\right) \int_{|x| \leq R_{0}^{2 s}}|U|^{2} d x \leq \frac{1}{2} \int_{|x| \leq R_{0}^{2 s+2}}|U|^{2} d x
$$

holds with such choices of $s$ and $R_{0}$.

It is time to use the three-ball inequality (4.1). To this end, we choose $r_{1}=$ $R_{0}^{2 k+2}, r_{2}=R_{0}^{2 k}$ and $r_{3}=R_{0}^{2 k-2}$ for $k \geq 1$ and require $R_{0}^{2} \leq \min \left\{(1 / 4 e)^{2}, R_{1}\right\}$. Thus (4.1) implies

$$
\begin{aligned}
& \int_{|x|<R_{0}^{2 k}}\left|U_{R_{0}^{2 k}}\right|^{2} d x / \int_{|x|<R_{0}^{2 k+2}}\left|U_{R_{0}^{2 k+2}}\right|^{2} d x \\
& \leq C^{1 / \tau}\left(\int_{|x|<R_{0}^{2 k-2}}\left|U_{R_{0}^{2 k-2}}\right|^{2} d x / \int_{|x|<R_{0}^{2 k}}\left|U_{R_{0}^{2 k}}\right|^{2} d x\right)^{a},
\end{aligned}
$$

where

$$
C=\max \left\{4 C_{0} R_{0}^{-4}, \exp \left(\beta_{0}\left(-1-4 \log R_{0}\right)\right)\right\} R_{0}^{-8 \tau}
$$

and

$$
\begin{aligned}
a=\frac{1-\tau}{\tau}=\frac{A}{B} & =\frac{\left(\log \left(r_{1} / r_{3}\right)-1\right)^{2}-\left(\log \left(r_{2} / r_{3}\right)\right)^{2}}{-1-2 \log \left(r_{2} / r_{3}\right)} \\
& =\frac{\left(4 \log R_{0}-1\right)^{2}-\left(2 \log R_{0}\right)^{2}}{-1-4 \log R_{0}} .
\end{aligned}
$$

It is not hard to see that

$$
\left\{\begin{array}{l}
1<C \leq C_{0} R_{0}^{-\beta_{1}}, \\
2<a \leq-4 \log R_{0},
\end{array}\right.
$$

where $\beta_{1}=32 \max \left\{1, \beta_{0}\right\}$ (note $\tau<1$ ). Combining (4.51) and using (4.50) recursively, we have that

$$
\begin{aligned}
& \int_{|x| \leq R_{0}^{2 s}}\left|U_{R_{0}^{2 s}}\right|^{2} d x / \int_{|x| \leq R_{0}^{2 s+2}}\left|U_{R_{0}^{2 s+2}}\right|^{2} d x \\
\leq & C^{1 / \tau}\left(\int_{|x|<R_{0}^{2 s-2}}\left|U_{R_{0}^{2 s-2}}\right|^{2} d x / \int_{|x|<R_{0}^{2 s}}\left|U_{R_{0}^{2 s}}\right|^{2} d x\right)^{a} \\
\leq & C^{\frac{a^{s-1}-1}{\tau(a-1)}}\left(\int_{|x|<R_{0}^{2}}\left|U_{R_{0}^{2}}\right|^{2} d x / \int_{|x|<R_{0}^{4}}\left|U_{R_{0}^{4}}\right|^{2} d x\right)^{a^{s-1}}
\end{aligned}
$$

for all $s \geq 1$. Now from the definition of $a$, we have $\tau=1 /(a+1)$ and thus

$$
\frac{a^{s-1}-1}{\tau(a-1)}=\frac{a+1}{a-1}\left(a^{s-1}-1\right) \leq 3 a^{s-1} .
$$


Then it follows from (4.52) that

$$
\begin{aligned}
& \int_{|x| \leq R_{0}^{2 s}}\left|U_{R_{0}^{2 s}}\right|^{2} d x / \int_{|x| \leq R_{0}^{2 s+2}}\left|U_{R_{0}^{2 s+2}}\right|^{2} d x \\
\leq & C^{3\left(-4 \log R_{0}\right)^{s-1}}\left(\int_{|x|<R_{0}^{2}}\left|U_{\left.R_{0}^{2}\right|^{2} d x /} \int_{|x|<R_{0}^{4}}\right| U_{\left.\left.R_{0}^{4}\right|^{2} d x\right)^{a^{s-1}}}\right. \\
\leq & \left(C_{0}^{3}\left(R_{0}\right)^{-3 \beta_{1}}\right)^{\left(-4 \log R_{0}\right)^{s-1}}\left(\int_{|x|<R_{0}^{2}}\left|U_{R_{0}^{2}}\right|^{2} d x / \int_{|x|<R_{0}^{4}}\left|U_{R_{0}^{4}}\right|^{2} d x\right)^{a^{s-1}} .
\end{aligned}
$$

Note that

$$
\begin{aligned}
& \int_{|x| \leq R_{0}^{2 s}}|U|^{2} d x \leq R_{0}^{-4 s} \int_{|x| \leq R_{0}^{2 s}}\left|U_{R_{0}^{2 s}}\right|^{2} d x, \\
& \left.\int_{|x| \leq R_{0}^{2 s+2}}\left|U_{\left.R_{0}^{2 s+2}\right|^{2}} d x \leq \int_{|x| \leq R_{0}^{2 s+2}}\right| U\right|^{2} d x .
\end{aligned}
$$

Thus, by (4.53) and (4.54), we can get that

$$
\begin{aligned}
& \exp \left(-c R_{0}^{-2 s}\right) \int_{|x| \leq R_{0}^{2 s}}|U|^{2} d x \\
\leq & \exp \left(-c R_{0}^{-2 s}\right) R_{0}^{-4 s}\left(C_{0}^{3}\left(R_{0}\right)^{-3 \beta_{1}}\right)^{\left(-4 \log R_{0}\right)^{s-1}} \\
& \left(\left.\int_{|x|<R_{0}^{2}}\left|U_{\left.R_{0}^{2}\right|^{2}} d x / \int_{|x|<R_{0}^{4}}\right| U_{R_{0}^{4}}\right|^{2} d x\right)^{a^{s-1}} \int_{|x| \leq R_{0}^{2 s+2}}|U|^{2} d x .
\end{aligned}
$$

Let $\mu=-\log R_{0}$, then if $R_{0}$ is sufficiently small, i.e., $\mu$ is sufficiently large, we can see that

$$
\frac{c}{4} \exp (2 \mu t)>4 t \mu+(4 \mu)^{t-1}\left(\log C_{0}^{3}+3 \beta_{1} \mu\right)
$$

for all $t \in \mathbb{N}$. In other words, we have that for $R_{0}$ small

$$
R_{0}^{-4 t}\left(C_{0}^{3} R_{0}^{-3 \beta_{1}}\right)^{\left(-4 \log R_{0}\right)^{t-1}}<\exp \left(c R_{0}^{-2 t} / 4\right)<(1 / 2) \exp \left(c R_{0}^{-2 t} / 2\right)
$$

for all $t \in \mathbb{N}$. We now fix a $R_{0} \leq \min \left\{1 / 4 e, \sqrt{R_{1}}\right\}$ so that (4.56) holds. The constants $m_{0}\left(R_{0}\right)$ and $j_{0}\left(R_{0}\right)$ are then fixed as well. It is a key step in our proof that we can find a universal constant $R_{0}$. After fixing $R_{0}$, we then define a number $t_{0}$, depending on $R_{0}$ and $U$, by

$$
\begin{aligned}
t_{0}= & \inf \{t \in \mathbb{R}: t \geq(\log 2-\log (a c) \\
& \left.\left.+\log \log \left(\int_{|x|<R_{0}^{2}}\left|U_{R_{0}^{2}}\right|^{2} d x / \int_{|x|<R_{0}^{4}}\left|U_{R_{0}^{4}}\right|^{2} d x\right)\right)\left(-2 \log R_{0}-\log a\right)^{-1}\right\} .
\end{aligned}
$$


By (4.51), one can easily check that $-2 \log R_{0}-\log a>0$ for all $R_{0} \leq 1 / 16$. With the choice of $t_{0}$, we can see that

$$
\left(\int_{|x|<R_{0}^{2}}\left|U_{R_{0}^{2}}\right|^{2} d x / \int_{|x|<R_{0}^{4}}\left|U_{R_{0}^{4}}\right|^{2} d x\right)^{a^{t-1}} \leq \exp \left(c R_{0}^{-2 t} / 2\right)
$$

for all $t \geq t_{0}$.

Let $s_{1}$ be the smallest positive integer such that $s_{1} \geq t_{0}$. If

$$
R_{0}^{2 s_{1}} \leq R_{j_{0}}=\left(\gamma\left(j_{0}+1 / 2\right)\right)^{-1},
$$

then we can find a $j_{1} \in \mathbb{N}$ with $j_{1} \geq j_{0}$ such that (4.47) holds, i.e.,

$$
R_{j_{1}+1}<R_{0}^{2 s_{1}} \leq R_{j_{1}}
$$

On the other hand, if

$$
R_{0}^{2 s_{1}}>R_{j_{0}}
$$

then we pick the smallest positive integer $s_{2}>s_{1}$ such that $R_{0}^{2 s_{2}} \leq R_{j_{0}}$ and thus we can also find a $j_{1} \in \mathbb{N}$ with $j_{1} \geq j_{0}$ for which (4.47) holds. We now define

$$
s=\left\{\begin{array}{llll}
s_{1} & \text { if } & (4.58) & \text { holds } \\
s_{2} & \text { if } & (4.59) & \text { holds }
\end{array}\right.
$$

It is important to note that with such an $s,(4.47)$ is satisfied for some $j_{1}$ and (4.56), (4.57) hold. Now we set $m_{1}=2+2\left(j_{1}+1 / 2\right)$ and $m=\left(m_{1}-2\right) / 2$. Combining (4.55), (4.56) and (4.57) yields that

$$
\begin{aligned}
& \exp \left(-c R_{0}^{-2 s}\right) \int_{|x| \leq R_{0}^{2 s}}|U|^{2} d x \\
& \leq \exp \left(-c R_{0}^{-2 s}\right) R_{0}^{-4 s}\left(C_{0}^{3}\left(R_{0}\right)^{-3 \beta_{1}}\right)^{\left(-3 \log R_{0}\right)^{s-1}} \\
& \left(\left.\int_{|x|<R_{0}^{2}}\left|U_{\left.R_{0}^{2}\right|^{2}} d x / \int_{|x|<R_{0}^{4}}\right| U_{R_{0}^{4}}\right|^{2} d x\right)^{a^{(s-1)}} \int_{|x| \leq R_{0}^{2 s+2}}|U|^{2} d x . \\
& \leq \frac{1}{2} \int_{|x| \leq R_{0}^{2 s+2}}|U|^{2} d x
\end{aligned}
$$

which is (4.49). Using (4.49) in (4.48), we have that

$$
\begin{aligned}
& \frac{1}{2}(2 \delta)^{-2 m-2} \int_{|x| \leq 2 \delta}|U|^{2} d x+\frac{1}{2}\left(R_{0}^{2} R_{j_{1}}\right)^{-2 m-2} \int_{|x| \leq R_{0}^{2 s+2}}|U|^{2} d x \\
& \leq\left(C^{\prime}+1\right)(\delta / 3)^{-2 m-2} \int_{|x| \leq \delta}|U|^{2} d x .
\end{aligned}
$$


It follows from (4.60) that

$$
\frac{1}{2\left(C^{\prime}+1\right)}\left(3 R_{0}^{2} R_{j_{1}}\right)^{-m_{1}} \int_{|x| \leq R_{0}^{2 s+2}}|U|^{2} d x \leq \delta^{-m_{1}} \int_{|x| \leq \delta}|U|^{2} d x
$$

and

$$
\frac{1}{2}(2 \delta)^{-2 m-2} \int_{|x| \leq 2 \delta}|U|^{2} d x \leq\left(C^{\prime}+1\right)(\delta / 3)^{-2 m-2} \int_{|x| \leq \delta}|U|^{2} d x
$$

which implies

$$
\int_{|x| \leq 2 \delta}|U|^{2} d x \leq \frac{1}{2\left(C^{\prime}+1\right)} 6^{m_{1}} \int_{|x| \leq \delta}|U|^{2} d x
$$

The estimates (4.61) and (4.62) are valid for all $\delta \leq R_{0}^{2 s+2} / 4$. Now we choose $R_{2}=$ $R_{0}$ in Theorem 4.4 and $R_{3}=R_{0}^{2 s+2} / 4$ in Theorem 4.5 . The proof is complete.

\subsection{Lipschitz propagation of smallness}

To study our inverse problem, we need to obtain three-ball inequalities in terms of $\sum_{i j}\left|e_{i j}^{\theta}(u)\right|^{2}+\rho_{0}^{2}\left|\partial_{i j}^{2} u_{3}\right|^{2}$ instead of $\left|u^{\prime}\right|^{2}+\left|u_{3}\right|^{2}$. To this end, the following Caccioppoli-type inequality is useful.

Lemma 4.11. Assume that $\lambda(x), \mu(x) \in L^{\infty}\left(B_{\rho}\right)$ satisfying (3.3) and there exists $K_{3}>0$ such that

$$
\|\lambda\|_{L^{\infty}\left(B_{\rho}\right)}+\|\mu\|_{L^{\infty}\left(B_{\rho}\right)}+\|\nabla \theta\|_{L^{\infty}\left(B_{\rho}\right)} \leq K_{3} .
$$

Let $\left(u^{\prime}, u_{3}\right) \in\left(H^{1}\left(B_{\rho}\right)\right)^{2} \times H^{2}\left(B_{\rho}\right)$ be a solution of $(1.1)$ in $B_{\rho}$. Then there exists a constant $C>0$, depending on $\delta_{0}, K_{3}$ such that

$$
\int_{B_{\rho / 2}} \sum_{i j}\left|e_{i j}^{\theta}(u)\right|^{2}+\rho_{0}^{2}\left|\partial_{i j}^{2} u_{3}\right|^{2} \leq \frac{C}{\rho^{2}} \int_{B_{\rho}}\left|u^{\prime}\right|^{2}+C\left(\frac{1}{\rho^{4}}+\frac{1}{\rho^{2}}\right) \int_{B_{\rho}}\left|u_{3}\right|^{2} .
$$

Proof. The proof of this lemma is adopted from [21]. Let $\eta \in C_{0}^{4}\left(B_{\rho}\right)$ with $0 \leq$ $\eta \leq 1$ and $\eta \equiv 1$ on $B_{\rho / 2}$ satisfying

$$
\sum_{|\alpha| \leq 3} \rho^{|\alpha|}\left|\partial^{\alpha} \eta\right| \leq C_{1} \quad \text { in } \quad B_{\rho}
$$

for some positive constant $C_{1}$. Multiplying the first equation of (1.1) by $\eta^{4} u^{\prime}$ and the second equation of (1.1) by $\eta^{4} u_{3}$ and performing integration by parts, we can get that

$$
\int_{B_{\rho}} n_{i j}^{\theta}(u) \partial_{j}\left(\eta^{4} u_{i}\right)+\int_{B_{\rho}} m_{i j}\left(u_{3}\right) \partial_{i j}^{2}\left(\eta^{4} u_{3}\right)+\int_{B_{\rho}} n_{i j}^{\theta}(u) \partial_{i} \theta \partial_{j}\left(\eta^{4} u_{3}\right)=0 .
$$


It is easy to see that (4.65) is equivalent to

$$
\begin{aligned}
& \int_{B_{\rho}} n_{i j}^{\theta}(u)\left[\eta^{4} \partial_{j} u_{i}+4\left(\partial_{j} \eta\right) \eta^{3} u_{i}\right] \\
& \quad+m_{i j}\left(u_{3}\right)\left[\eta^{4} \partial_{i j}^{2} u_{3}+8\left(\partial_{j} \eta\right) \eta^{3} \partial_{i} u_{3}+\left(\partial_{i j} \eta^{4}\right) u_{3}\right] \\
& \quad+n_{i j}^{\theta}(u) \partial_{i} \theta\left[\eta^{4} \partial_{j} u_{3}+4\left(\partial_{j} \eta\right) \eta^{3} u_{3}\right]=0
\end{aligned}
$$

It follows from (4.66) that

$$
\begin{aligned}
& \int_{B_{\rho}} \eta^{4} \sum_{i j}\left(n_{i j}^{\theta}(u) e_{i j}^{\theta}(u)+m_{i j}\left(u_{3}\right) \partial_{i j}^{2} u_{3}\right) \\
& =-4 \int_{B_{\rho}} n_{i j}^{\theta}(u)\left(\partial_{j} \eta\right) \eta^{3} u_{i}-8 \int_{B_{\rho}} m_{i j}\left(u_{3}\right)\left(\partial_{j} \eta\right) \eta^{3} \partial_{i} u_{3} \\
& \quad-\int_{B_{\rho}} m_{i j}\left(u_{3}\right)\left(\partial_{i j}^{2} \eta^{4}\right) u_{3} \\
& \quad-4 \int_{B_{\rho}} n_{i j}^{\theta}(u)\left(\partial_{i} \theta\right)\left(\partial_{j} \eta\right) \eta^{3} u_{3} .
\end{aligned}
$$

Observe that

$$
\left|\int_{B_{\rho}} n_{i j}^{\theta}(u)\left(\partial_{j} \eta\right) \eta^{3} u_{i}\right| \leq \frac{\varepsilon}{2} \int_{B_{\rho}} \eta^{4} \sum_{i j}\left|n_{i j}^{\theta}\right|^{2}+\frac{C_{2}}{\varepsilon \rho^{2}} \int_{B_{\rho}}\left|u^{\prime}\right|^{2}
$$

for some $C_{2}>0$, depending on $C_{1}$. Likewise, we can obtain that

$$
\left|\int_{B_{\rho}} m_{i j}\left(u_{3}\right)\left(\partial_{i j}^{2} \eta^{4}\right) u_{3}\right| \leq \frac{\varepsilon}{2} \int_{B_{\rho}} \eta^{4} \sum_{i j}\left|m_{i j}\left(u_{3}\right)\right|^{2}+\frac{C_{3}}{\varepsilon \rho^{4}} \int_{B_{\rho}}\left|u_{3}\right|^{2}
$$

for some $C_{3}>0$, and

$$
\left|\int_{B_{\rho}} n_{i j}^{\theta}(u)\left(\partial_{i} \theta\right)\left(\partial_{j} \eta\right) \eta^{3} u_{3}\right| \leq \frac{\varepsilon}{2} \int_{B_{\rho}} \eta^{4} \sum_{i j}\left|n_{i j}^{\theta}(u)\right|^{2}+\frac{C_{4}}{\varepsilon \rho^{2}} \int_{B_{\rho}}\left|u_{3}\right|^{2}
$$

for some $C_{4}>0$, also depending on $K_{3}$. Finally, we have

$$
\begin{aligned}
\left|\int_{B_{\rho}} m_{i j}\left(u_{3}\right)\left(\partial_{j} \eta\right) \eta^{3} \partial_{i} u_{3}\right| \leq & \frac{\varepsilon}{2} \int_{B_{\rho}} \eta^{4} \sum_{i j}\left|m_{i j}\left(u_{3}\right)\right|^{2} \\
& +\frac{1}{2 \varepsilon} \int_{B_{\rho}} \eta^{2} \sum_{i j}\left(\partial_{j} \eta \partial_{i} u_{3}\right)^{2} .
\end{aligned}
$$


Using the same computations on page 10-11 of [21], we get that

$$
\int_{B_{\rho}} \eta^{2} \sum_{i j}\left(\partial_{j} \eta \partial_{i} u_{3}\right)^{2} \leq \frac{C_{5}}{\rho^{4}}\left(1+\frac{1}{\varepsilon^{2}}\right) \int_{B_{\rho}}\left|u_{3}\right|^{2}+\frac{\varepsilon^{2}}{2} \int_{B_{\rho}} \eta^{4} \sum_{i j}\left|\partial_{i j}^{2} u_{3}\right|^{2}
$$

for some $C_{5}>0$. Putting (4.67)-(4.72) together and taking $\varepsilon$ sufficiently small, we immediately arrive at the desired estimate (4.63).

Remark 4.12. If $\rho \leq 1$, then (4.63) can be written as

$$
\int_{B_{\rho / 2}} \sum_{i j}\left|e_{i j}^{\theta}(u)\right|^{2}+\rho_{0}^{2}\left|\partial_{i j}^{2} u_{3}\right|^{2} \leq \frac{C}{\rho^{4}} \int_{B_{\rho}}\left|u^{\prime}\right|^{2}+\left|u_{3}\right|^{2} .
$$

Our aim here is to derive another version of the three sphere inequality. We will make use of arguments introduced in [5]. For any scalar or vector valued function $f$, we denote $(f)_{r}=\frac{1}{\left|B_{r}\right|} \int_{B_{r}} f$. Then we define an operator $T:\left(H^{1}\left(B_{R}\right)\right)^{2} \times$ $H^{2}\left(B_{R}\right) \rightarrow\left(H^{1}\left(B_{R}\right)\right)^{2} \times H^{2}\left(B_{R}\right)$ by $T u=T\left(u^{\prime}, u_{3}\right)=\left(v^{\prime}(x ; r), v_{3}(x ; r)\right)$, where

$$
\begin{aligned}
v^{\prime}(x ; r)= & \left(u^{\prime}\right)_{r}+\frac{1}{2}\left(\nabla u^{\prime}-\left(\nabla u^{\prime}\right)^{t}\right)_{r} x \\
& +\frac{1}{2}\left[(\nabla \theta)_{r} \otimes\left(\nabla u_{3}\right)_{r}-\left((\nabla \theta)_{r} \otimes\left(\nabla u_{3}\right)_{r}\right)^{t}\right] x \\
& -\left(\theta-(\theta)_{r}\right)\left(\nabla u_{3}\right)_{r}
\end{aligned}
$$

and

$$
v_{3}(x ; r)=\left(u_{3}\right)_{r}+\left(\nabla u_{3}\right)_{r} \cdot x .
$$

Here the tensor product of two vectors $\xi$ and $\eta$ is defined as

$$
\xi \otimes \eta=\left(\begin{array}{ll}
\xi_{1} \eta_{1} & \xi_{1} \eta_{2} \\
\xi_{2} \eta_{1} & \xi_{2} \eta_{2}
\end{array}\right)
$$

Note that $(x)_{r}=0$.

We now denote the space

$$
\mathcal{R}=\left\{w=\left(w^{\prime}, w_{3}\right) \mid w^{\prime}=a+W x-\theta c, w_{3}=b+c \cdot x\right\}
$$

where $a, c$ are two-dimensional vectors, $b$ is a scalar, and $W$ is a $2 \times 2$ skewsymmetric matrix. It is readily seen that $T u=u$ for all $u \in \mathcal{R}$. Denote $L u=$ $\left(\left(e_{i j}^{\theta}(u)\right)_{1 \leq i \leq 2,1 \leq j \leq 2},\left(\partial_{i j}^{2} u_{3}\right)_{1 \leq i \leq 2,1 \leq j \leq 2}\right)$. It is also easy to check that $\mathcal{R}$ is the null space of $L$. We need to compute the norm of $T$. Recall that

$$
\begin{aligned}
& \left\|v^{\prime}\right\|_{H^{1}\left(B_{R}\right)}^{2}+\left\|v_{3}\right\|_{H^{2}\left(B_{R}\right)}^{2} \\
= & \frac{1}{R^{2}} \int_{B_{R}}\left|v^{\prime}\right|^{2}+\int_{B_{R}}\left|\nabla v^{\prime}\right|^{2}+\frac{1}{R^{2}} \int_{B_{R}}\left|v_{3}\right|^{2}+\int_{B_{R}}\left|\nabla v_{3}\right|^{2}+R^{2} \int_{B_{R}}\left|\nabla^{2} v_{3}\right|^{2} .
\end{aligned}
$$


In view of (4.74), (4.75) and the assumption on $\theta$, we obtain that

$$
\|T\| \leq C\left(1+\frac{R^{2}}{r^{2}}+\frac{1}{r^{2}}\right)^{1 / 2}
$$

with an absolute constant $C>0$. Assume that $B_{R} \subset \Omega$ and so (3.2) holds on $B_{R}$. Now if we take $r=R$, then $\left(u^{\prime}-v^{\prime}(\cdot ; R), u_{3}-v_{3}(\cdot ; R)\right)$ satisfies the normalization conditions (3.9) with $\Omega$ be replaced by $B_{R}$ and therefore (3.10) becomes

$$
\left\|u^{\prime}-v^{\prime}(\cdot ; R)\right\|_{H^{1}\left(B_{R}\right)}^{2}+\left\|u_{3}-v_{3}(\cdot ; R)\right\|_{H^{2}\left(B_{R}\right)}^{2} \leq C \int_{B_{R}} \sum_{i j}\left|e_{i j}^{\theta}(u)\right|^{2}+R^{2}\left|\partial_{i j}^{2} u_{3}\right|^{2},
$$

where $C$ depends on $A_{2}$. Using Lemma 2.1 in [5], we conclude that

$$
\begin{gathered}
\left\|u^{\prime}-v^{\prime}(\cdot ; r)\right\|_{H^{1}\left(B_{R}\right)}^{2}+\left\|u_{3}-v_{3}(\cdot ; r)\right\|_{H^{2}\left(B_{R}\right)}^{2} \\
\leq C(1+\|T\|)^{2} \int_{B_{R}} \sum_{i j}\left|e_{i j}^{\theta}(u)\right|^{2}+R^{2}\left|\partial_{i j}^{2} u_{3}\right|^{2} \\
\leq C\left(1+\frac{R^{2}}{r^{2}}+\frac{1}{r^{2}}\right) \int_{B_{R}} \sum_{i j}\left|e_{i j}^{\theta}(u)\right|^{2}+R^{2}\left|\partial_{i j}^{2} u_{3}\right|^{2} .
\end{gathered}
$$

In particular, we have that

$$
\begin{aligned}
& \int_{B_{R}}\left|u^{\prime}-v^{\prime}(\cdot ; r)\right|^{2}+\left|u_{3}-v_{3}(\cdot ; r)\right|^{2} \\
& \leq C R^{2}\left(1+\frac{R^{2}}{r^{2}}+\frac{1}{r^{2}}\right)\left(1+\frac{R^{2}}{\rho_{0}^{2}}\right) \int_{B_{R}} \sum_{i j}\left|e_{i j}^{\theta}(u)\right|^{2}+\rho_{0}^{2}\left|\partial_{i j}^{2} u_{3}\right|^{2} .
\end{aligned}
$$

We now prove the following three-ball inequalities.

Theorem 4.13. Assume that $\bar{R}_{0}$ and $R_{1}$ are given in Theorem 4.1. If $0<r_{1}<$ $r_{2}<2 r_{2}<r_{3} \leq \min \left\{\rho_{0} \bar{R}_{0}, 1\right\}$ and $r_{1} / r_{3}<2 r_{2} / r_{3}<R_{1}$, then

$$
\begin{aligned}
& \int_{B_{r_{2}}} \sum_{i j}\left|e_{i j}^{\theta}(u)\right|^{2}+\rho_{0}^{2}\left|\partial_{i j}^{2} u_{3}\right|^{2} \\
& \leq \frac{C}{r_{2}^{6}}\left(\frac{r_{3}}{r_{1}}\right)^{2-2 \tau}\left(\int_{B_{r_{1}}} \sum_{i j}\left|e_{i j}^{\theta}(u)\right|^{2}+\rho_{0}^{2}\left|\partial_{i j}^{2} u_{3}\right|^{2}\right)^{\tau} \\
& \quad \times\left(\int_{B_{r_{3}}} \sum_{i j}\left|e_{i j}^{\theta}(u)\right|^{2}+\rho_{0}^{2}\left|\partial_{i j}^{2} u_{3}\right|^{2}\right)^{1-\tau}
\end{aligned}
$$

for $\left(u^{\prime}, u_{3}\right) \in\left(H^{1}\left(B_{\rho_{0} \bar{R}_{0}}\right)\right)^{2} \times H^{3}\left(B_{\rho_{0} \bar{R}_{0}}\right)$ satisfying (1.1), where $C>0$ and $0<$ $\tau<1$ depend on $r_{1} / r_{3}, r_{2} / r_{3}, \delta_{0}, A_{2}$. 
Proof. Let $\tilde{u}^{\prime}=u^{\prime}-v^{\prime}\left(x ; r_{1}\right), \tilde{u}_{3}=u_{3}-v_{3}\left(x ; r_{1}\right)$. Note that $\left(\tilde{u}^{\prime}, \tilde{u}_{3}\right)$ satisfies the normalization condition (3.9) on $B_{r_{1}}$. Recall that $\bar{R}_{0} \leq 1$. Now combining (4.73), (4.76), (4.2), and (3.10) implies that

$$
\begin{aligned}
& \int_{B_{r_{2}}} \sum_{i j}\left|e_{i j}^{\theta}(u)\right|^{2}+\rho_{0}^{2}\left|\partial_{i j}^{2} u_{3}\right|^{2} \\
= & \int_{B_{r_{2}}} \sum_{i j}\left|e_{i j}^{\theta}(\tilde{u})\right|^{2}+\rho_{0}^{2}\left|\partial_{i j}^{2} \tilde{u}_{3}\right|^{2} \\
\leq & \frac{C}{r_{2}^{4}} \int_{B_{2 r_{2}}}\left|\tilde{u}^{\prime}\right|^{2}+\left|\tilde{u}_{3}\right|^{2} \\
\leq & \left.\frac{C^{\prime}}{r_{2}^{6}}\left(\int_{B_{r_{1}}}\left|\tilde{u}^{\prime}\right|^{2}+\left|\tilde{u}_{3}\right|^{2}\right)^{\tau}\left(\int_{B_{r_{3}}}\left|\tilde{u}^{\prime}\right|^{2}+\left|\tilde{u}_{3}\right|^{2}\right)^{1-\tau}\right)^{\tau}\left(r_{3}^{2}\left(1+\frac{r_{3}^{2}}{r_{1}^{2}}+\frac{1}{r_{1}^{2}}\right)\left(1+\frac{r_{3}^{2}}{\rho_{0}^{2}}\right)\right)^{1-\tau} \\
\leq & \frac{C^{\prime \prime}}{r_{2}^{6}}\left(r_{1}^{2}\left(1+\frac{1}{r_{1}^{2}}\right)\left(1+\frac{r_{1}^{2}}{2}\right)\right. \\
& \times\left(\int_{B_{r_{1}}} \sum_{i j}\left|e_{i j}^{\theta}(u)\right|^{2}+\rho_{0}^{2}\left|\partial_{i j}^{2} u_{3}\right|^{2}\right)^{\tau}\left(\int_{B_{r_{3}}} \sum_{i j}\left|e_{i j}^{\theta}(u)\right|^{2}+\rho_{0}^{2}\left|\partial_{i j}^{2} u_{3}\right|^{2}\right)^{1-\tau} \\
\leq & C^{\prime \prime \prime \prime} \frac{1}{r_{2}^{6}}\left(\frac{r_{3}}{r_{1}}\right)^{2-2 \tau} \\
& \times\left(\int_{B_{r_{1}}} \sum_{i j}\left|e_{i j}^{\theta}(u)\right|^{2}+\rho_{0}^{2}\left|\partial_{i j}^{2} u_{3}\right|^{2}\right)^{\tau}\left(\int_{B_{r_{3}}} \sum_{i j}\left|e_{i j}^{\theta}(u)\right|^{2}+\rho_{0}^{2}\left|\partial_{i j}^{2} u_{3}\right|^{2}\right)^{1-\tau} .
\end{aligned}
$$

A key ingredient in solving our inverse problem is a continuation estimate from the interior for the solution $u$ of (3.4), (3.5). To do this, we need some assumptions the coupled field $(\widehat{T}, \widehat{M})$. We assume that $(\widehat{T}, \widehat{M})$ satisfies

$$
\operatorname{supp}(\widehat{T}, \widehat{M}) \subset \Gamma_{0},
$$

where $\Gamma_{0}$ is an open subarc of $\partial \Omega$ with

$$
\left|\Gamma_{0}\right| \leq\left(1-\gamma_{0}\right)|\partial \Omega|
$$

for some $\gamma_{0}>0$. We first prove the following lemma.

Lemma 4.14. Let $\Omega$ be a bounded domain in $\mathbb{R}^{2}$ with $C^{2,1}$ boundary $\partial \Omega$ characterized by constants $A_{0}$ and $\rho_{0}$. Assume that $\lambda, \mu \in L^{\infty}(\Omega)$ satisfy (3.3), $\nabla \theta \in$ $L^{\infty}(\Omega)$, and

$$
\|\lambda\|_{L^{\infty}(\Omega)}+\|\mu\|_{L^{\infty}(\Omega)}+\|\nabla \theta\|_{L^{\infty}(\Omega)} \leq A_{2}
$$


for some $A_{2}>0$. Let $\left(u^{\prime}, u_{3}\right) \in\left(H^{1}(\Omega)\right)^{2} \times H^{2}(\Omega)$ be the unique weak solution of (3.4), (3.5) satisfying (3.9), with $(\widehat{T}, \widehat{M}) \in H^{-1 / 2}(\partial \Omega) \times H^{-1 / 2}(\partial \Omega)$ satisfying (4.78), (4.79), and (3.8). Then we have

$$
\|(\widehat{T}, \widehat{M})\|_{\left(H^{-1 / 2}(\partial \Omega)\right)^{3}} \leq C\left(\int_{\Omega} \sum_{i j}\left|e_{i j}^{\theta}(u)\right|^{2}+\rho_{0}^{2}\left|\partial_{i j}^{2} u_{3}\right|^{2}\right)^{1 / 2},
$$

where $C$ depends on $\delta_{0}, A_{0}, A_{1}, A_{2}, \gamma_{0}$.

Proof. We follow the arguments used in the proof of [20, Lemma 7.1]. For any $(f, g) \in\left(H^{1 / 2}(\partial \Omega)\right)^{3}$, one can find $\left(v^{\prime}, v_{3}\right) \in\left(H^{1}(\Omega)\right)^{2} \times H^{2}(\Omega)$ such that $\left.v^{\prime}\right|_{\partial \Omega}=$ $f,\left.v_{3}\right|_{\partial \Omega}=0,\left.\partial_{\nu} v_{3}\right|_{\partial \Omega}=g$ and

$$
\left\|\left(v^{\prime}, v_{3}\right)\right\|_{\left(H^{1}(\Omega)\right)^{2} \times H^{2}(\Omega)} \leq C\left\|\left(f, \rho_{0} g\right)\right\|_{\left(H^{1 / 2}(\partial \Omega)\right)^{3}},
$$

where $C$ depends on $A_{0}$ and $A_{1}$. In view of the weak formulation of the solution, we can compute

$$
\begin{aligned}
& \int_{\partial \Omega} \frac{1}{\rho_{0}} \widehat{T} \cdot f+\widehat{M}_{\nu} g=\frac{1}{\rho_{0}} \int_{\partial \Omega} \widehat{T} \cdot v^{\prime}+\widehat{M}_{\nu} \rho_{0} g \\
& =\frac{1}{\rho_{0}} \int_{\partial \Omega} \widehat{T} \cdot v^{\prime}+\rho_{0} \widehat{M}_{\nu} \partial_{\nu} v_{3}+\rho_{0} \partial_{s} \widehat{M}_{\tau} v_{3} \\
& =\frac{1}{\rho_{0}^{2}} \int_{\Omega} \sum_{i j}\left(\rho_{0}^{2} n_{i j}^{\theta}(u) e_{i j}^{\theta}(v)+\rho_{0}^{4} m_{i j}\left(u_{3}\right) \partial_{i j}^{2} v_{3}\right) \\
& \leq C\left(\frac{1}{\rho_{0}^{2}} \int_{\Omega} \sum_{i j} \rho_{0}^{2}\left|e_{i j}^{\theta}(u)\right|^{2}+\rho_{0}^{4}\left|\partial_{i j}^{2} u_{3}\right|^{2}\right)^{1 / 2} \\
& \quad \times\left(\frac{1}{\rho_{0}^{2}} \int_{\Omega} \sum_{i j} \rho_{0}^{2}\left|e_{i j}^{\theta}(v)\right|^{2}+\rho_{0}^{4}\left|\partial_{i j}^{2} v_{3}\right|^{2}\right)^{1 / 2} \\
& \leq C\left(\frac{1}{\rho_{0}^{2}} \int_{\Omega} \sum_{i j} \rho_{0}^{2}\left|e_{i j}^{\theta}(u)\right|^{2}+\rho_{0}^{4}\left|\partial_{i j}^{2} u_{3}\right|^{2}\right)^{1 / 2}\left\|\left(v^{\prime}, v_{3}\right)\right\|_{\left(H^{1}(\Omega)\right)^{2} \times H^{2}(\Omega)} \\
& \leq C\left(\frac{1}{\rho_{0}^{2}} \int_{\Omega} \sum_{i j} \rho_{0}^{2}\left|e_{i j}^{\theta}(u)\right|^{2}+\rho_{0}^{4}\left|\partial_{i j}^{2} u_{3}\right|^{2}\right)^{1 / 2}\left\|\left(f, \rho_{0} g\right)\right\|_{\left(H^{1 / 2}(\partial \Omega)\right)^{3},}
\end{aligned}
$$

where $C$ depends on $\delta_{0}, A_{0}, A_{1}, A_{2}$. Consequently, we obtain

$$
\left\|\left(\widehat{T}, \widehat{M}_{\nu}\right)\right\|_{\left(H^{-1 / 2}(\partial \Omega)\right)^{3}} \leq C\left(\int_{\Omega} \sum_{i j}\left|e_{i j}^{\theta}(u)\right|^{2}+\rho_{0}^{2}\left|\partial_{i j}^{2} u_{3}\right|^{2}\right)^{1 / 2} .
$$


On the other hand, for any given $g \in H^{1 / 2}(\partial \Omega)$, let $h \in H^{3 / 2}\left(\Gamma_{0}\right)$ satisfy $\partial_{s} h=$ $g$ on $\Gamma_{0}$ and $\|h\|_{H^{3 / 2}\left(\Gamma_{0}\right)} \leq C\left\|\rho_{0} g\right\|_{H^{1 / 2}\left(\Gamma_{0}\right)} \leq C\left\|\rho_{0} g\right\|_{H^{1 / 2}(\partial \Omega)}$, where $C$ here depends on $A_{0}$ and $A_{1}$. Now let $\tilde{h} \in H^{3 / 2}(\partial \Omega)$ be such that $\tilde{h}=h$ on $\Gamma_{0}$ and $\|\tilde{h}\|_{H^{3 / 2}(\partial \Omega)} \leq C\|h\|_{H^{3 / 2}\left(\Gamma_{0}\right)}$, where $C$ depends on $A_{0}, A_{1}, \gamma_{0}$. Moreover, let $v_{3} \in$ $H^{2}(\Omega)$ satisfy $v_{3}=\tilde{h}, \partial_{\nu} v_{3}=0$ on $\partial \Omega$ and $\left\|v_{3}\right\|_{H^{2}(\Omega)} \leq C\|\tilde{h}\|_{H^{3 / 2}(\partial \Omega)}$, where $C$ depends on $A_{0}$ and $A_{1}$. Let $f \in\left(H^{1 / 2}(\partial \Omega)\right)^{2}$ be the same function given above. Now we can derive that

$$
\begin{aligned}
& \int_{\partial \Omega} \frac{1}{\rho_{0}} \widehat{T} \cdot(-f)+\widehat{M}_{\tau} g=\frac{1}{\rho_{0}} \int_{\Gamma_{0}} \widehat{T} \cdot(-f)+\rho_{0} \widehat{M}_{\tau} \partial_{s} h \\
= & -\frac{1}{\rho_{0}} \int_{\Gamma_{0}} \widehat{T} \cdot f+\rho_{0} \partial_{s} \widehat{M}_{\tau} \tilde{h} \\
= & -\frac{1}{\rho_{0}} \int_{\partial \Omega} \widehat{T} \cdot f+\rho_{0} \partial_{s} \widehat{M}_{\tau} v_{3}+\rho_{0} \widehat{M}_{\nu} \partial_{\nu} v_{3} \\
\leq & C\left(\frac{1}{\rho_{0}^{2}} \int_{\Omega} \sum_{i j} \rho_{0}^{2}\left|e_{i j}^{\theta}(u)\right|^{2}+\rho_{0}^{4}\left|\partial_{i j}^{2} u_{3}\right|^{2}\right)^{1 / 2}\left\|\left(f, \rho_{0} g\right)\right\|_{\left(H^{1 / 2}(\partial \Omega)\right)^{3}}
\end{aligned}
$$

with $C$ depending on $\delta_{0}, A_{0}, A_{1}, A_{2}, \gamma_{0}$, which implies

$$
\|(\widehat{T}, \widehat{M})\|_{\left(H^{-1 / 2}(\partial \Omega)\right)^{3}} \leq C\left(\int_{\Omega} \sum_{i j}\left|e_{i j}^{\theta}(u)\right|^{2}+\rho_{0}^{2}\left|\partial_{i j}^{2} u_{3}\right|^{2}\right)^{1 / 2} .
$$

Finally, combining (4.82) and (4.83) leads to (4.80).

We are now ready to prove the following theorem.

Theorem 4.15 (Lipschitz propagation of smallness). Assume that $\Omega$ is a bounded domain having boundary $\partial \Omega \in C^{4,1}$ with constants $A_{0}, \rho_{0}$. Let $\lambda, \mu \in C^{1,1}(\bar{\Omega})$ satisfy (3.3) and $\bar{\theta} \in C^{2,1}(\bar{\Omega})$ satisfy (3.22) and (3.23) hold. Let $u \in\left(H^{1}(\Omega)\right)^{2} \times$ $H^{2}(\Omega)$ be the weak solution of (3.4), (3.5) satisfying (3.9) with Neumann boundary condition $(\widehat{T}, \widehat{M}) \in\left(H^{1 / 2}(\partial \Omega)\right)^{2} \times H^{3 / 2}(\partial \Omega)$ satisfying (3.8), (4.78), (4.79). Then for every $\rho>0$ and every $x \in \Omega_{\frac{7}{\vartheta}} \rho \rho_{0}$, we have

$$
\int_{B_{\rho \rho_{0}}(x)} \sum_{i j}\left|e_{i j}^{\theta}(u)\right|^{2}+\rho_{0}^{2}\left|\partial_{i j}^{2} u_{3}\right|^{2} \geq C_{\rho} \int_{\Omega} \sum_{i j}\left|e_{i j}^{\theta}(u)\right|^{2}+\rho_{0}^{2}\left|\partial_{i j}^{2} u_{3}\right|^{2},
$$

where $C_{\rho}$ depends on $A_{0}, A_{1}, A_{2}, \delta_{0}, \gamma_{0}, \rho$, and $\|(\widehat{T}, \widehat{M})\|_{\left(L^{2}(\partial \Omega)\right)^{2} \times H^{1 / 2}(\partial \Omega)}^{2} /$ $\|(\widehat{T}, \widehat{M})\|_{\left(H^{-1 / 2}(\partial \Omega)\right)^{3}}$. Here $\vartheta=R_{1}$ and $R_{1}$ is the constant given in Theorem 4.1 .

Proof. There is no restriction to take $\rho_{0}=1$. The general case can be proved by a simple scaling argument. Note that $\Omega_{\frac{7 \rho}{\vartheta}}$ is connected for all $0<\rho \leq \zeta$, where $\zeta$ 
depends on $\delta_{0}, A_{0}, A_{2}$. It suffices to prove the result for small $\rho$. We now choose a $\rho$ such that $7 \rho / \vartheta \leq \bar{R}_{0}$. Let $y \in \Omega_{\frac{7 \rho}{\vartheta}}$ and $\gamma(t)$ be an arc in $\Omega_{\frac{7 \rho}{\vartheta}}$ joining $y$ and $x$. We now define $\left\{x_{i}\right\}, i=1, \cdots, L$, as follows: $x_{1}=x, x_{i+1}=\gamma\left(t_{i}\right)$ with $t_{i}=\max \left\{t|| \gamma(t)-x_{i} \mid=2 \rho\right\}$ if $\left|x_{i}-y\right|>2 \rho$, otherwise, let $i=L$ and stop the process. By construction, we can see that the spheres $B_{\rho}\left(x_{i}\right)$ are pairwise disjoint and $\left|x_{i+1}-x_{i}\right|=2 \rho$ for $i=1, \cdots, L-1,\left|x_{L}-y\right| \leq 2 \rho$.

Since $x_{i} \in \Omega_{\frac{7 \rho}{\vartheta}}$, we use the three-sphere inequality (4.77) with $x=x_{i}, r_{1}=\rho$, $r_{2}=3 \rho, r_{3}=\frac{7 \rho}{\vartheta} \leq \bar{R}_{0}<1$ for $i=1, \cdots, L-1$ to obtain

$$
\frac{\int_{B_{\rho}\left(x_{i+1}\right)} \sum_{i j}\left|e_{i j}^{\theta}(u)\right|^{2}+\left|\partial_{i j}^{2} u_{3}\right|^{2}}{\int_{\Omega} \sum_{i j}\left|e_{i j}^{\theta}(u)\right|^{2}+\left|\partial_{i j}^{2} u_{3}\right|^{2}} \leq C\left(\frac{1}{\rho}\right)^{6}\left(\frac{\int_{B_{\rho}\left(x_{i}\right)} \sum_{i j}\left|e_{i j}^{\theta}(u)\right|^{2}+\left|\partial_{i j}^{2} u_{3}\right|^{2}}{\int_{\Omega} \sum_{i j}\left|e_{i j}^{\theta}(u)\right|^{2}+\left|\partial_{i j}^{2} u_{3}\right|^{2}}\right)^{\tau},
$$

where $C>0$ depends on $\delta_{0}, A_{2}$. Induction on $i$ implies

$$
\frac{\int_{B_{\rho}(y)} \sum_{i j}\left|e_{i j}^{\theta}(u)\right|^{2}+\left|\partial_{i j}^{2} u_{3}\right|^{2}}{\int_{\Omega} \sum_{i j}\left|e_{i j}^{\theta}(u)\right|^{2}+\left|\partial_{i j}^{2} u_{3}\right|^{2}} \leq C^{\frac{1}{1-\tau}}\left(\frac{1}{\rho}\right)^{\frac{6}{1-\tau}}\left(\frac{\int_{B_{\rho}(x)} \sum_{i j}\left|e_{i j}^{\theta}(u)\right|^{2}+\left|\partial_{i j}^{2} u_{3}\right|^{2}}{\int_{\Omega} \sum_{i j}\left|e_{i j}^{\theta}(u)\right|^{2}+\left|\partial_{i j}^{2} u_{3}\right|^{2}}\right)^{L} .
$$

Note that $L \leq|\Omega| /\left(\pi \rho^{2}\right) \leq A_{1} / \pi$.

Let us now cover $\Omega_{\frac{8 \rho}{\vartheta}}$ with internally nonoverlapping closed cubes of side $\ell=\sqrt{2} \rho / \vartheta$. It is clear that any such cube is contained in a sphere of radius $\rho$ with center in $\Omega_{\frac{7 \rho}{\vartheta}}$ and the number of such cube is controlled by $N=2|\Omega| \vartheta^{2} /\left(4 \rho^{2}\right)$. It follows from (4.85) that

$$
\frac{\int_{\Omega_{8 \rho / \vartheta}} \sum_{i j}\left|e_{i j}^{\theta}(u)\right|^{2}+\left|\partial_{i j}^{2} u_{3}\right|^{2}}{\int_{\Omega} \sum_{i j}\left|e_{i j}^{\theta}(u)\right|^{2}+\left|\partial_{i j}^{2} u_{3}\right|^{2}} \leq C\left(\frac{1}{\rho}\right)^{\frac{8-2 \tau}{1-\tau}}\left(\frac{\int_{B_{\rho}(x)} \sum_{i j}\left|e_{i j}^{\theta}(u)\right|^{2}+\left|\partial_{i j}^{2} u_{3}\right|^{2}}{\int_{\Omega} \sum_{i j}\left|e_{i j}^{\theta}(u)\right|^{2}+\left|\partial_{i j}^{2} u_{3}\right|^{2}}\right)^{\tau^{L}} .
$$

Here $C$ depends on $\delta_{0}, A_{2},|\Omega|$.

Now we want to estimate the left-hand side of (4.86) from below by a positive constant. Obviously, we have

$$
\frac{\int_{\Omega_{8 \rho / \vartheta}} \sum_{i j}\left|e_{i j}^{\theta}(u)\right|^{2}+\left|\partial_{i j}^{2} u_{3}\right|^{2}}{\int_{\Omega} \sum_{i j}\left|e_{i j}^{\theta}(u)\right|^{2}+\left|\partial_{i j}^{2} u_{3}\right|^{2}}=1-\frac{\int_{\Omega \backslash \Omega_{8 \rho / \vartheta}} \sum_{i j}\left|e_{i j}^{\theta}(u)\right|^{2}+\left|\partial_{i j}^{2} u_{3}\right|^{2}}{\int_{\Omega} \sum_{i j}\left|e_{i j}^{\theta}(u)\right|^{2}+\left|\partial_{i j}^{2} u_{3}\right|^{2}} .
$$

It suffices to show that there exists $\bar{\rho}>0$ such that

$$
\frac{\int_{\Omega \backslash \Omega_{8 \rho / \vartheta}} \sum_{i j}\left|e_{i j}^{\theta}(u)\right|^{2}+\left|\partial_{i j}^{2} u_{3}\right|^{2}}{\int_{\Omega} \sum_{i j}\left|e_{i j}^{\theta}(u)\right|^{2}+\left|\partial_{i j}^{2} u_{3}\right|^{2}} \leq \frac{1}{2},
$$

for every $\rho, 0<\rho \leq \bar{\rho}$. The proposition, then, follows from (4.86) and (4.88). 
By Hölder's inequality and Sobolev's inequality

$$
\|w\|_{L^{4}(\Omega)}^{2} \leq C\|w\|_{H^{1 / 2}(\Omega)}^{2}
$$

with $C$ depending on $A_{0}, A_{1}$, we have

$$
\begin{aligned}
& \int_{\Omega \backslash \Omega_{8 \rho / \vartheta}} \sum_{i j}\left|e_{i j}^{\theta}(u)\right|^{2}+\left|\partial_{i j}^{2} u_{3}\right|^{2} \\
& \leq\left|\Omega \backslash \Omega_{8 \rho / \vartheta}\right|^{1 / 2} \sum_{i j}\left(\int_{\Omega \backslash \Omega_{8 \rho / \vartheta}}\left(\left|e_{i j}^{\theta}(u)\right|^{2}+\left|\partial_{i j}^{2} u_{3}\right|^{2}\right)^{2}\right)^{1 / 2} \\
& \leq \sqrt{2}\left|\Omega \backslash \Omega_{8 \rho / \vartheta}\right|^{1 / 2} \sum_{i j}\left(\left(\int_{\Omega \backslash \Omega_{8 \rho / \vartheta}}\left|e_{i j}^{\theta}(u)\right|^{4}\right)^{1 / 2}+\left(\int_{\Omega \backslash \Omega_{8 \rho / \vartheta}}\left|\partial_{i j}^{2} u_{3}\right|^{4}\right)^{1 / 2}\right) \\
& \leq C\left|\Omega \backslash \Omega_{8 \rho / \vartheta}\right|^{1 / 2}\left\|\left(u^{\prime}, u_{3}\right)\right\|_{\left(H^{3 / 2}(\Omega)\right)^{2} \times H^{5 / 2}(\Omega)}^{2} .
\end{aligned}
$$

Interpolating the global estimates (3.18) and (3.24) yields

$$
\begin{aligned}
& \left\|\left(u^{\prime}, u_{3}\right)\right\|_{\left(H^{3 / 2}(\Omega)\right)^{2} \times H^{5 / 2}(\Omega)} \\
& \leq\left\|\left(u^{\prime}, u_{3}\right)\right\|_{\left(H^{3 / 2}(\Omega)\right)^{2} \times H^{3}(\Omega)} \\
& \leq C\|(\widehat{T}, \widehat{M})\|_{\left(L^{2}(\partial \Omega)\right)^{2} \times H^{1 / 2}(\partial \Omega)}
\end{aligned}
$$

where $C$ depends on $A_{0}, A_{1}, A_{2}, \delta_{0}$.

Following the argument of [6] (see [6, A.3] for details), there exists a positive constant $C$, depending on $A_{0}, A_{1}, A_{2}, \delta_{0}$, such that

$$
\left|\Omega \backslash \Omega_{8 \rho / \vartheta}\right| \leq C \rho .
$$

It follows from (4.89), (4.90), and (4.91) that

$$
\int_{\Omega \backslash \Omega_{8 \rho / \vartheta}} \sum_{i j}\left|e_{i j}^{\theta}(u)\right|^{2}+\left|\partial_{i j}^{2} u_{3}\right|^{2} \leq C \rho^{1 / 2}\|(\widehat{T}, \widehat{M})\|_{\left(L^{2}(\partial \Omega)\right)^{2} \times H^{1 / 2}(\partial \Omega)}^{2} .
$$

From (4.80), we can obtain that

$$
\frac{\int_{\Omega \backslash \Omega_{8 \rho / \vartheta}} \sum_{i j}\left|e_{i j}^{\theta}(u)\right|^{2}+\left|\partial_{i j}^{2} u_{3}\right|^{2}}{\int_{\Omega} \sum_{i j}\left|e_{i j}^{\theta}(u)\right|^{2}+\left|\partial_{i j}^{2} u_{3}\right|^{2}} \leq C \rho^{1 / 2} \frac{\|(\widehat{T}, \widehat{M})\|_{\left(L^{2}(\partial \Omega)\right)^{2} \times H^{1 / 2}(\partial \Omega)}^{2}}{\|(\widehat{T}, \widehat{M})\|_{\left(H^{-1 / 2}(\partial \Omega)\right)^{3}}},
$$

where $C$ depends on $A_{0}, A_{1}, A_{2}, \delta_{0}, \gamma_{0}$. Finally, we can choose $\bar{\rho}$, depending on $A_{0}, A_{1}, A_{2}, \delta_{0}, \gamma_{0}$, and $\|(\widehat{T}, \widehat{M})\|_{\left(L^{2}(\partial \Omega)\right)^{2} \times H^{1 / 2}(\partial \Omega)}^{2} /\|(\widehat{T}, \widehat{M})\|_{\left(H^{-1 / 2}(\partial \Omega)\right)^{3}}$, such that

$$
\frac{\int_{\Omega \backslash \Omega_{8 \rho / \vartheta}} \sum_{i j}\left|e_{i j}^{\theta}(u)\right|^{2}+\left|\partial_{i j}^{2} u_{3}\right|^{2}}{\int_{\Omega} \sum_{i j}\left|e_{i j}^{\theta}(u)\right|^{2}+\left|\partial_{i j}^{2} u_{3}\right|^{2}} \leq \frac{1}{2}
$$

for all $0<\rho<\bar{\rho}$. The proof now is complete. 


\section{The inverse problem}

In this section, we would like to study the problem of estimating the size of inclusion embedded in a shallow shell by one boundary measurement. Let $\Omega \subset \mathbb{R}^{2}$ be an open bounded domain with boundary $\partial \Omega$, which is of class $C^{4,1}$ with constants $A_{0}, \rho_{0}$. Assume that (3.1) holds. Now let $D$ be a possibly disconnected measurable subdomain of $\Omega$ satisfying

$$
\operatorname{dist}(D, \partial \Omega) \geq d_{0} \rho_{0}
$$

for some given constant $d_{0}$. Let $\lambda, \mu \in C^{1,1}(\bar{\Omega})$ satisfy (3.3) and $\bar{\theta} \in C^{2,1}(\bar{\Omega})$ satisfy (3.22). Besides, assume that the estimate (3.23) holds. For measurable functions $\lambda_{0}, \mu_{0}$, we define

$$
\tilde{\lambda}=\lambda+\chi_{D} \lambda_{0} \quad \text { and } \quad \tilde{\mu}=\mu+\chi_{D} \mu_{0},
$$

where $\chi_{D}$ is the characteristic function of $D$. To guarantee the well-posedness of the forward problem, we assume

$$
0<\tilde{\delta}_{0} \leq \tilde{\lambda} \quad \text { and } \quad \tilde{\delta}_{0} \leq \tilde{\mu} \quad \forall x \in \Omega .
$$

To describe the jump condition, we introduce some shorthand notations. We set

$$
a=\frac{4 \lambda \mu}{\lambda+2 \mu}, b=4 \mu, c=\frac{4 \lambda \mu}{3(\lambda+2 \mu)}, d=\frac{4 \mu}{3}
$$

and the corresponding $\tilde{a}, \tilde{b}, \tilde{c}, \tilde{d}$ replacing $\lambda, \mu$ with $\tilde{\lambda}, \tilde{\mu}$ respectively. We assume the following condition on the jump at the interface $\partial D$. There exists a constant $k_{0}>0$ such that

$$
(\tilde{f}-f) \leq k_{0} f \quad \forall x \in \partial D,
$$

where $f=a, b, c, d$ and $\tilde{f}=\tilde{a}, \tilde{b}, \tilde{c}, \tilde{d}$. On the prescribed boundary field $(\widehat{T}, \widehat{M})$, we assume

$$
(\widehat{T}, \widehat{M}) \in\left(H^{1 / 2}(\partial \Omega)\right)^{2} \times H^{3 / 2}(\partial \Omega) \quad \text { and } \operatorname{supp}(\widehat{T}, \widehat{M}) \subset \Gamma_{0},
$$

where $\Gamma_{0}$ is an open subarc of $\partial \Omega$ with

$$
\left|\Gamma_{0}\right| \leq\left(1-\gamma_{0}\right)|\partial \Omega|
$$

for some $\gamma_{0}>0$ and satisfies the compatibility condition (3.8). We consider two boundary value problems. Let $u=\left(u^{\prime}, u_{3}\right)$ satisfy

$$
\left\{\begin{array}{l}
\partial_{j} n_{i j}^{\theta}(u)=0 \text { in } \Omega, \\
\partial_{i j}^{2} m_{i j}\left(u_{3}\right)-\partial_{j}\left(n_{i j}^{\theta}(u) \partial_{i} \theta\right)=0 \text { in } \Omega,
\end{array}\right.
$$


with boundary conditions

$$
\left\{\begin{array}{l}
n_{i j}^{\theta}(u) v_{j}=\rho_{0}^{-1} \widehat{T}_{i}, \\
m_{i j}\left(u_{3}\right) v_{i} v_{j}=\widehat{M}_{\nu}, \\
\left(\partial_{i} m_{i j}\left(u_{3}\right)-n_{i j}^{\theta}(u) \partial_{i} \theta\right) v_{j}+\partial_{s}\left(m_{i j}\left(u_{3}\right) v_{i} \tau_{j}\right)=-\partial_{s} \widehat{M}_{\tau} .
\end{array}\right.
$$

Next we let $\tilde{u}=\left(\widetilde{u}^{\prime}, \tilde{u}_{3}\right)$ satisfy

$$
\left\{\begin{array}{l}
\partial_{j} \widetilde{n}_{i j}^{\theta}(\widetilde{u})=0 \text { in } \Omega, \\
\partial_{i j}^{2} \widetilde{m}_{i j}\left(\widetilde{u}_{3}\right)-\partial_{j}\left(\widetilde{n}_{i j}^{\theta}(\widetilde{u}) \partial_{i} \theta\right)=0 \text { in } \Omega,
\end{array}\right.
$$

with boundary conditions

$$
\left\{\begin{array}{l}
\tilde{n}_{i j}^{\theta}\left(\widetilde{u}_{)}\right) v_{j}=\rho_{0}^{-1} \widehat{T}_{i}, \\
\tilde{m}_{i j}\left(\widetilde{u}_{3}\right) v_{i} v_{j}=\widehat{M}_{\nu}, \\
\left(\partial_{i} \tilde{m}_{i j}\left(\widetilde{u}_{3}\right)-\tilde{n}_{i j}^{\theta}(\widetilde{u}) \partial_{i} \theta\right) v_{j}+\partial_{s}\left(\tilde{m}_{i j}\left(\widetilde{u}_{3}\right) v_{i} \tau_{j}\right)=-\partial_{s} \widehat{M}_{\tau} .
\end{array}\right.
$$

To ensure the uniqueness of the solution, we impose the normalization conditions (3.9). Let

$$
\begin{aligned}
\widetilde{W} & =\int_{\partial \Omega} \rho_{0}^{-1} \widehat{T} \cdot \tilde{u}^{\prime}+\widehat{M}_{\nu} \partial_{\nu} \tilde{u}_{3}+\partial_{s} \widehat{M}_{\tau} \tilde{u}_{3}, \\
W & =\int_{\partial \Omega} \rho_{0}^{-1} \widehat{T} \cdot u^{\prime}+\widehat{M}_{\nu} \partial_{\nu} u_{3}+\partial_{s} \widehat{M}_{\tau} u_{3} \\
& =\int_{\Omega} \sum_{i j} n_{i j}^{\theta}(u) e_{i j}^{\theta}(u)+m_{i j}\left(u_{3}\right) \partial_{i j}^{2} u_{3},
\end{aligned}
$$

represent the work exerted by the boundary field when the inclusion is present or absent, respectively. For $r>0$ we shall use the notation

$$
D_{r}=\{x \in D: \operatorname{dist}(x, \partial D)>r\} .
$$

We can now state our main result.

Theorem 5.1. Suppose that all the hypotheses stated in this section hold. Moreover, we assume $\rho_{0}<1$. Let $D$ be an inclusion satisfying the following fatness condition

$$
\left|D_{h_{1} \rho_{0}}\right| \geq \frac{1}{2}|D|
$$

for a given positive constant $h_{1}$. Then we have the estimate

$$
C_{1} \rho_{0}^{2}\left|\frac{W-\widetilde{W}}{W}\right| \leq|D| \leq C_{2} \rho_{0}^{2}\left|\frac{W-\widetilde{W}}{W}\right|,
$$

where $C_{1}$ depends on $A_{0}, A_{1}, A_{2}, d_{0}, k_{0}$ and $\delta_{0}$ and $C_{2}$ depends on $A_{0}, A_{1}, A_{2}, \delta_{0}$, $\gamma_{0}, d_{0}, h_{1}$ and the ratio

$$
\|(\widehat{M}, \widehat{T})\|_{\left(L^{2}(\partial \Omega)\right)^{2} \times H^{1 / 2}(\partial \Omega)} /\|(\widehat{M}, \widehat{T})\|_{\left(H^{-1 / 2}(\partial \Omega)\right)^{3}} .
$$


The key ingredients in the proof of Theorem 5.1 are the energy estimate for the Neumann problems (5.6)-(5.7), (5.8)-(5.9), and the Lipschitz propagation of smallness.

Lemma 5.2. Let $(\widehat{M}, \widehat{T}) \in L^{2}(\partial \Omega)$ satisfy (5.4), (5.5) and (3.8). Let $\lambda, \mu, \tilde{\lambda}, \tilde{\mu} \in$ $L^{\infty}(\Omega)$ satisfy (3.3) and (5.3). Let $u, \tilde{u} \in\left(H^{1}(\Omega)\right)^{2} \times H^{2}(\Omega)$ solutions to (5.6)(5.7) and (5.8)-(5.9) respectively. Then there exist positive constants $\tilde{C}_{1}, \tilde{C}_{2}$ depending on $A_{0}, A_{1}, A_{2}, \delta_{0}, k_{0}$ such that

$$
\tilde{C}_{1} \int_{D} \sum_{i j}\left|e_{i j}^{\theta}(u)\right|^{2}+\rho_{0}^{2}\left|\partial_{i j}^{2} u_{3}\right|^{2} \leq W-\tilde{W} \leq \tilde{C}_{2} \int_{D} \sum_{i j}\left|e_{i j}^{\theta}(u)\right|^{2}+\rho_{0}^{2}\left|\partial_{i j}^{2} u_{3}\right|^{2} .
$$

With the help of Lemma 5.2 and Theorem 4.15, we first prove Theorem 5.1.

Proof of Theorem 5.1. By the interior regularity theorem and the Sobolev embedding, we have that

$$
\left\|\sum_{i j}\left|e_{i j}^{\theta}(u)\right|^{2}+\rho_{0}^{2}\left|\partial_{i j}^{2} u_{3}\right|^{2}\right\|_{L^{\infty}(D)} \leq \frac{C}{\rho_{0}^{2}}\left(\left\|u^{\prime}\right\|_{H^{1}(\Omega)}^{2}+\left\|u_{3}\right\|_{H^{2}(\Omega)}^{2}\right),
$$

with $C$ depending on $A_{2}, d_{0}, \delta_{0}$. From (5.14), Proposition 3.1, (5.10), we obtain that

$$
\left\|\sum_{i j}\left|e_{i j}^{\theta}(u)\right|^{2}+\rho_{0}^{2}\left|\partial_{i j}^{2} u_{3}\right|^{2}\right\|_{L^{\infty}(D)} \leq \frac{C}{\rho_{0}^{2}} W,
$$

where $C$ depends on $A_{0}, A_{1}, A_{2}, d_{0}, \delta_{0}$. The lower bound on $|D|$ in (5.12) follows from the right-hand side of (5.13) and from (5.15).

Let us prove the upper bound for $|D|$. Let $\varepsilon=\min \left\{2 d_{0} \vartheta / 7, h_{1} / \sqrt{2}\right\}$ and let us cover $D_{h_{1} \rho_{0}}$ with internally non overlapping closed squares $Q_{l}$ of side $\varepsilon \rho_{0}$ for $l=1, \ldots, L$. By choice of $\varepsilon$ the squares $Q_{l}$ are contained in $D$. Let $\bar{l}$ be such that

$$
\int_{Q_{\bar{l}}} \sum_{i j}\left|e_{i j}^{\theta}(u)\right|^{2}+\rho_{0}^{2}\left|\partial_{i j}^{2} u_{3}\right|^{2}=\min _{l} \int_{Q_{l}} \sum_{i j}\left|e_{i j}^{\theta}(u)\right|^{2}+\rho_{0}^{2}\left|\partial_{i j}^{2} u_{3}\right|^{2} .
$$

Noticing that $\left|D_{h_{1} \rho_{0}}\right| \leq L \varepsilon^{2} \rho_{0}^{2}$, we have

$$
\begin{aligned}
\int_{D} \sum_{i j}\left|e_{i j}^{\theta}(u)\right|^{2}+\rho_{0}^{2}\left|\partial_{i j}^{2} u_{3}\right|^{2} & \geq \int_{\cup_{l=1}^{L} Q_{l}} \sum_{i j}\left|e_{i j}^{\theta}(u)\right|^{2}+\rho_{0}^{2}\left|\partial_{i j}^{2} u_{3}\right|^{2} \\
& \geq L \int_{Q_{\bar{l}}} \sum_{i j}\left|e_{i j}^{\theta}(u)\right|^{2}+\rho_{0}^{2}\left|\partial_{i j}^{2} u_{3}\right|^{2} \\
& \geq \frac{\left|D_{h_{1} \rho_{0}}\right|}{\rho_{0}^{2} \varepsilon^{2}} \int_{Q_{\bar{l}}} \sum_{i j}\left|e_{i j}^{\theta}(u)\right|^{2}+\rho_{0}^{2}\left|\partial_{i j}^{2} u_{3}\right|^{2} .
\end{aligned}
$$


Let $\bar{x}$ be the center of $Q_{\bar{l}}$. From (5.16), estimate (4.84) with $x=\bar{x}$ and $\rho=\varepsilon / 2$, and hypothesis (5.11), we conclude that

$$
\int_{D} \sum_{i j}\left|e_{i j}^{\theta}(u)\right|^{2}+\rho_{0}^{2}\left|\partial_{i j}^{2} u_{3}\right|^{2} \geq \frac{K|D|}{\rho_{0}^{2}} W,
$$

where $K$ is a positive constant depending on $A_{0}, A_{1}, A_{2}, d_{0}, \delta_{0}, \gamma_{0}, h_{1}$ and $\|(\widehat{M}, \widehat{T})\|_{\left(L^{2}(\partial \Omega)\right)^{2} \times H^{1 / 2}(\partial \Omega)} /\|(\widehat{M}, \widehat{T})\|_{\left(H^{-1 / 2}(\partial \Omega)\right)^{3}}$. The upper bound for $D$ follows from the left-hand side of (5.13) and from (5.17).

To end this section, we give a proof of Lemma 5.2.

Proof of Lemma 5.2. Let $w=\left(w^{\prime}, w_{3}\right)$. From the first equation of (5.6), we have that

$$
\int_{\Omega} n_{i j}^{\theta}(u) \partial_{j} w_{i}^{\prime}=\int_{\partial \Omega} \rho_{0}^{-1} \widehat{T}_{i} w_{i}^{\prime} .
$$

On the other hand, by the second equation of (5.6), the integration by parts leads to

$$
\begin{aligned}
& \int_{\Omega} m_{i j}\left(u_{3}\right) \partial_{i j}^{2} w_{3}+n_{i j}^{\theta}(u) \partial_{i} \theta \partial_{j} w_{3} \\
& =\int_{\partial \Omega}-\partial_{i} m_{i j}\left(u_{3}\right) v_{j} w_{3}+m_{i j}\left(u_{3}\right) v_{i} \partial_{j} w_{3}+n_{i j}^{\theta}(u) \partial_{i} \theta v_{j} w_{3} .
\end{aligned}
$$

Replacing $\partial_{j} w_{3}$ by $v_{j} \partial_{v} w_{3}+\tau_{j} \partial_{s} w_{3}$ in (5.19) gives

$$
\begin{aligned}
& \int_{\Omega} m_{i j}\left(u_{3}\right) \partial_{i j}^{2} w_{3}+n_{i j}^{\theta}(u) \partial_{i} \theta \partial_{j} w_{3} \\
& =\int_{\partial \Omega}-\partial_{i} m_{i j}\left(u_{3}\right) v_{j} w_{3}+m_{i j}\left(u_{3}\right) v_{i}\left(v_{j} \partial_{\nu} w_{3}+\tau_{j} \partial_{s} w_{3}\right) \\
& \quad+n_{i j}^{\theta}(u) \partial_{i} \theta v_{j} w_{3} \\
& =\int_{\partial \Omega}-\partial_{i} m_{i j}\left(u_{3}\right) v_{j} w_{3}-\partial_{s}\left(m_{i j}\left(u_{3}\right) v_{i} \tau_{j}\right) w_{3}+n_{i j}^{\theta}(u) \partial_{i} \theta v_{j} w_{3} \\
& \quad+m_{i j}\left(u_{3}\right) v_{i} v_{j} \partial_{\nu} w_{3} \\
& =\int_{\partial \Omega} \partial_{s} \widehat{M}_{\tau} w_{3}+\widehat{M}_{\nu} \partial_{\nu} w_{3} .
\end{aligned}
$$

Thus, by combining (5.18) and (5.19), we get

$$
\begin{aligned}
& \int_{\Omega} n_{i j}^{\theta}(u) \partial_{j} w_{i}^{\prime}+m_{i j}\left(u_{3}\right) \partial_{i j}^{2} w_{3}+n_{i j}^{\theta}(u) \partial_{i} \theta \partial_{j} w_{3} \\
& =\int_{\partial \Omega} \rho_{0}^{-1} \widehat{T}_{i} w_{i}^{\prime}+\partial_{s} \widehat{M}_{\tau} w_{3}+\widehat{M}_{\nu} \partial_{\nu} w_{3}
\end{aligned}
$$


Likewise, we can deduce

$$
\begin{aligned}
& \int_{\Omega} \tilde{n}_{i j}^{\theta}(\widetilde{u}) \partial_{j} w_{i}^{\prime}+\tilde{m}_{i j}\left(\widetilde{u}_{3}\right) \partial_{i j}^{2} w_{3}+\widetilde{n}_{i j}^{\theta}(\widetilde{u}) \partial_{i} \theta \partial_{j} w_{3} \\
& =\int_{\partial \Omega} \rho_{0}^{-1} \widehat{T}_{i} w_{i}^{\prime}+\partial_{s} \widehat{M}_{\tau} w_{3}+\widehat{M}_{\nu} \partial_{\nu} w_{3}
\end{aligned}
$$

and therefore,

$$
\begin{aligned}
& \int_{\Omega} n_{i j}^{\theta}(u) \partial_{j} w_{i}^{\prime}+m_{i j}\left(u_{3}\right) \partial_{i j}^{2} w_{3}+n_{i j}^{\theta}(u) \partial_{i} \theta \partial_{j} w_{3} \\
= & \int_{\Omega} \tilde{n}_{i j}^{\theta}(\tilde{u}) \partial_{j} w_{i}^{\prime}+\tilde{m}_{i j}\left(\tilde{u}_{3}\right) \partial_{i j}^{2} w_{3}+\tilde{n}_{i j}^{\theta}(\tilde{u}) \partial_{i} \theta \partial_{j} w_{3} .
\end{aligned}
$$

In turn, we obtain

$$
\begin{aligned}
& \int_{\Omega} \tilde{n}_{i j}^{\theta}(\tilde{u}-u) \partial_{j} w_{i}^{\prime}+\tilde{m}_{i j}\left(\tilde{u}_{3}-u_{3}\right) \partial_{i j}^{2} w_{3}+\tilde{n}_{i j}^{\theta}(\tilde{u}-u) \partial_{i} \theta \partial_{j} w_{3} \\
& =\int_{\Omega}\left(n_{i j}^{\theta}-\tilde{n}_{i j}^{\theta}\right)(u) \partial_{j} w_{i}^{\prime}+\left(m_{i j}-\tilde{m}_{i j}\right)(u) \partial_{i j}^{2} w_{3} \\
& \quad+\left(n_{i j}^{\theta}-\tilde{n}_{i j}^{\theta}\right)(u) \partial_{i} \theta \partial_{j} w_{3} .
\end{aligned}
$$

Substituting $w=\tilde{u}$ into (5.23), we get that

$$
\begin{aligned}
& \int_{\Omega} \tilde{n}_{i j}^{\theta}(\tilde{u}-u) \partial_{j} \widetilde{u}_{i}^{\prime}+\tilde{m}_{i j}\left(\tilde{u}_{3}-u_{3}\right) \partial_{i j}^{2} \tilde{u}_{3}+\tilde{n}_{i j}^{\theta}(\tilde{u}-u) \partial_{i} \theta \partial_{j} \tilde{u}_{3} \\
& =\int_{\Omega}\left(n_{i j}^{\theta}-\tilde{n}_{i j}^{\theta}\right)(u) \partial_{j} \tilde{u}_{i}^{\prime}+\left(m_{i j}-\tilde{m}_{i j}\right)(u) \partial_{i j}^{2} \tilde{u}_{3} \\
& \quad+\left(n_{i j}^{\theta}-\tilde{n}_{i j}^{\theta}\right)(u) \partial_{i} \theta \partial_{j} \tilde{u}_{3} .
\end{aligned}
$$

By straightforward computations, we can see that

$$
\begin{aligned}
& \int_{\Omega} \tilde{n}_{i j}^{\theta}(\tilde{u}-u) \partial_{j} \tilde{u}_{i}^{\prime}+\tilde{m}_{i j}\left(\tilde{u}_{3}-u_{3}\right) \partial_{i j}^{2} \tilde{u}_{3}+\tilde{n}_{i j}^{\theta}(\tilde{u}-u) \partial_{i} \theta \partial_{j} \tilde{u}_{3} \\
= & \int_{\partial \Omega} \rho_{0}^{-1} \widehat{T}_{i}\left(\widetilde{u}_{i}^{\prime}-u_{i}^{\prime}\right)+\partial_{s} \widehat{M}_{\tau}\left(\widetilde{u}_{3}-u_{3}\right)+\widehat{M}_{\nu} \partial_{v}\left(\tilde{u}_{3}-u_{3}\right)
\end{aligned}
$$

and it follows from (5.24) that

$$
\begin{aligned}
& \int_{\Omega}\left(n_{i j}^{\theta}-\tilde{n}_{i j}^{\theta}\right)(u) \partial_{j} \widetilde{u}_{i}^{\prime}+\left(m_{i j}-\tilde{m}_{i j}\right)(u) \partial_{i j}^{2} \widetilde{u}_{3}+\left(n_{i j}^{\theta}-\tilde{n}_{i j}^{\theta}\right)(u) \partial_{i} \theta \partial_{j} \tilde{u}_{3} . \\
& =\int_{\partial \Omega} \rho_{0}^{-1} \widehat{T}_{i}\left(\widetilde{u}_{i}^{\prime}-u_{i}^{\prime}\right)+\partial_{s} \widehat{M}_{\tau}\left(\widetilde{u}_{3}-u_{3}\right)+\widehat{M}_{\nu} \partial_{\nu}\left(\widetilde{u}_{3}-u_{3}\right) .
\end{aligned}
$$


Now replacing $w=\tilde{u}-u$ in (5.23) and using (5.25), we obtain that

$$
\begin{aligned}
& \int_{\Omega} \tilde{n}_{i j}^{\theta}(\tilde{u}-u) \partial_{j}\left(\tilde{u}_{i}^{\prime}-u_{i}^{\prime}\right)+\tilde{m}_{i j}\left(\tilde{u}_{3}-u_{3}\right) \partial_{i j}^{2}\left(\tilde{u}_{3}-u_{3}\right) \\
& \quad+\tilde{n}_{i j}^{\theta}(\tilde{u}-u) \partial_{i} \theta \partial_{j}\left(\tilde{u}_{3}-u_{3}\right) \\
& =\int_{\Omega}\left(n_{i j}^{\theta}-\tilde{n}_{i j}^{\theta}\right)(u) \partial_{j}\left(\tilde{u}_{i}^{\prime}-u_{i}^{\prime}\right)+\left(m_{i j}-\tilde{m}_{i j}\right)(u) \partial_{i j}^{2}\left(\tilde{u}_{3}-u_{3}\right) \\
& \quad+\left(n_{i j}^{\theta}-\tilde{n}_{i j}^{\theta}\right)(u) \partial_{i} \theta \partial_{j}\left(\tilde{u}_{3}-u_{3}\right) \\
& =\int_{\partial \Omega} \rho_{0}^{-1} \widehat{T}_{i}\left(\widetilde{u}_{i}^{\prime}-u_{i}^{\prime}\right)+\partial_{s} \widehat{M}_{\tau}\left(\widetilde{u}_{3}-u_{3}\right)+\widehat{M}_{\nu} \partial_{\nu}\left(\widetilde{u}_{3}-u_{3}\right) \\
& \quad+\int_{D}\left(\tilde{n}_{i j}^{\theta}-n_{i j}^{\theta}\right)(u) \partial_{j} u_{i}^{\prime}+\left(\tilde{m}_{i j}-m_{i j}\right)(u) \partial_{i j}^{2} u_{3} \\
& \quad+\left(\widetilde{n}_{i j}^{\theta}-n_{i j}^{\theta}\right)(u) \partial_{i} \theta \partial_{j} u_{3} .
\end{aligned}
$$

Exchanging the role of $\tilde{u}$ and $u$, we can deduce that

$$
\begin{aligned}
& \int_{\Omega} n_{i j}^{\theta}(u-\widetilde{u}) \partial_{j}\left(u_{i}^{\prime}-\widetilde{u}_{i}^{\prime}\right)+m_{i j}\left(u_{3}-\widetilde{u}_{3}\right) \partial_{i j}^{2}\left(u_{3}-\widetilde{u}_{3}\right) \\
& \quad+n_{i j}^{\theta}(u-\widetilde{u}) \partial_{i} \theta \partial_{j}\left(u_{3}-\widetilde{u}_{3}\right) \\
& =\int_{\partial \Omega} \rho_{0}^{-1} \widehat{T}_{i}\left(u_{i}^{\prime}-\widetilde{u}_{i}^{\prime}\right)+\partial_{s} \widehat{M}_{\tau}\left(u_{3}-\widetilde{u}_{3}\right)+\widehat{M}_{\nu} \partial_{v}\left(u_{3}-\widetilde{u}_{3}\right) \\
& \quad+\int_{D}\left(n_{i j}^{\theta}-\widetilde{n}_{i j}^{\theta}\right)(\widetilde{u}) \partial_{j} \widetilde{u}_{i}^{\prime} \\
& \quad+\left(m_{i j}-\tilde{m}_{i j}\right)(\widetilde{u}) \partial_{i j}^{2} \widetilde{u}_{3}+\left(n_{i j}^{\theta}-\widetilde{n}_{i j}^{\theta}\right)(\widetilde{u}) \partial_{i} \theta \partial_{j} \tilde{u}_{3} .
\end{aligned}
$$

Finally, plugging $w=\tilde{u}$ into (5.21) and $w=u$ into (5.22), respectively, we have that

$$
\begin{aligned}
& \int_{D}\left(\tilde{n}_{i j}^{\theta}-n_{i j}^{\theta}\right)(\widetilde{u}) \partial_{j} u_{i}+\left(\tilde{m}_{i j}-m_{i j}\right)\left(\tilde{u}_{3}\right) \partial_{i j}^{2} u_{3}+\left(\tilde{n}_{i j}^{\theta}-n_{i j}^{\theta}\right)(\widetilde{u}) \partial_{i} \theta \partial_{j} u_{3} \\
& =\int_{\partial \Omega} \rho_{0}^{-1} \widehat{T}_{i}\left(u_{i}^{\prime}-\tilde{u}_{i}^{\prime}\right)+\partial_{s} \widehat{M}_{\tau}\left(u_{3}-\widetilde{u}_{3}\right)+\widehat{M}_{\nu} \partial_{\nu}\left(u_{3}-\tilde{u}_{3}\right) .
\end{aligned}
$$

The following identity is useful in our further arguments. Let $a, b, c$ and $d$ be any functions. It is easy to compute that

$$
\begin{aligned}
& \left(a e_{k k}^{\theta}(w) \delta_{i j}+b e_{i j}^{\theta}(w)\right) \partial_{j} w_{i}+\left(c\left(\Delta w_{3}\right) \delta_{i j}+d \partial_{i j}^{2} w_{3}\right) \partial_{i j}^{2} w_{3} \\
& +\left(a e_{k k}^{\theta}(w) \delta_{i j}+b e_{i j}^{\theta}(w)\right) \partial_{i} \theta \partial_{j} w_{3} \\
= & a\left|\nabla \cdot w^{\prime}+\nabla \theta \cdot \nabla w_{3}\right|^{2}+b \sum_{i j}\left|e_{i j}^{\theta}(w)\right|^{2}+c\left|\Delta w_{3}\right|^{2}+d \sum_{i j}\left|\partial_{i j}^{2} w_{3}\right|^{2}
\end{aligned}
$$


Now let $a, b, c, d$ be given in (5.2) and $\tilde{a}, \tilde{b}, \tilde{c}, \tilde{d}$ be defined similarly. Putting $w=$ $\tilde{u}-u$, using (5.29), (5.26), we get that

$$
\begin{aligned}
W-\tilde{W} \leq & \int_{D}\left(\tilde{n}_{i j}^{\theta}-n_{i j}^{\theta}\right)(u) \partial_{j} u_{i}^{\prime}+\left(\tilde{m}_{i j}-m_{i j}\right)(u) \partial_{i j}^{2} u_{3}+\left(\tilde{n}_{i j}^{\theta}-n_{i j}^{\theta}\right)(u) \partial_{i} \theta \partial_{j} u_{3} \\
= & \int_{D}(\widetilde{a}-a)\left|\nabla \cdot u^{\prime}+\nabla \theta \cdot \nabla u_{3}\right|^{2}+(\tilde{b}-b) \sum_{i j}\left|e_{i j}^{\theta}(u)\right|^{2} \\
& +(\tilde{c}-c)\left|\Delta u_{3}\right|^{2}+(\tilde{d}-d) \sum_{i j}\left|\partial_{i j}^{2} u_{3}\right|^{2} .
\end{aligned}
$$

On the other hand, for any $\varepsilon>0$, one can easily compute that

$$
\begin{aligned}
& \int_{D}(\tilde{a}-a)\left|\nabla \cdot u^{\prime}+\nabla \theta \cdot \nabla u_{3}\right|^{2}+(\tilde{b}-b) \sum_{i j}\left|e_{i j}^{\theta}(u)\right|^{2}+(\tilde{c}-c)\left|\Delta u_{3}\right|^{2} \\
& \quad+(\tilde{d}-d) \sum_{i j}\left|\partial_{i j}^{2} u_{3}\right|^{2} \\
& \leq\left(1+\varepsilon^{-1}\right) \int_{D}(\tilde{a}-a)\left|\nabla \cdot\left(\tilde{u}^{\prime}-u^{\prime}\right)+\nabla \theta \cdot \nabla\left(\tilde{u}_{3}-u_{3}\right)\right|^{2} \\
& \quad+(\tilde{b}-b) \sum_{i j}\left|e_{i j}^{\theta}(\tilde{u}-u)\right|^{2}+(\widetilde{c}-c)\left|\Delta\left(\tilde{u}_{3}-u_{3}\right)\right|^{2} \\
& \quad+(\tilde{d}-d) \sum_{i j}\left|\partial_{i j}^{2}\left(\tilde{u}_{3}-u_{3}\right)\right|^{2}+(1+\varepsilon) \int_{D}(\tilde{a}-a)\left|\nabla \cdot \tilde{u}^{\prime}+\nabla \theta \cdot \nabla \tilde{u}_{3}\right|^{2} \\
& \quad+(\tilde{b}-b) \sum_{i j}\left|e_{i j}^{\theta}(\tilde{u})\right|^{2}+(\tilde{c}-c)\left|\Delta \tilde{u}_{3}\right|^{2}+(\tilde{d}-d) \sum_{i j}\left|\partial_{i j}^{2} \tilde{u}_{3}\right|^{2} .
\end{aligned}
$$

By (5.3) we can choose $\varepsilon_{*}>0$ such that

$$
\frac{1+\varepsilon_{*}^{-1}}{1+\varepsilon_{*}}=\frac{1}{k_{0}} .
$$

Therefore, from (5.31) and (5.29), we deduce that

$$
\begin{aligned}
& \int_{D}(\widetilde{a}-a)\left|\nabla \cdot u^{\prime}+\nabla \theta \cdot \nabla u_{3}\right|^{2}+(\tilde{b}-b) \sum_{i j}\left|e_{i j}^{\theta}(u)\right|^{2}+(\widetilde{c}-c)\left|\Delta u_{3}\right|^{2} \\
& \quad+(\widetilde{d}-d) \sum_{i j}\left|\partial_{i j}^{2} u_{3}\right|^{2} \\
& \leq\left(1+\varepsilon_{*}\right)\left(\int_{D} n_{i j}^{\theta}(u-\widetilde{u}) \partial_{j}\left(u_{i}^{\prime}-\widetilde{u}_{i}^{\prime}\right)+m_{i j}\left(u_{3}-\widetilde{u}_{3}\right) \partial_{i j}^{2}\left(u_{3}-\tilde{u}_{3}\right)\right. \\
& \quad+n_{i j}^{\theta}(u-\widetilde{u}) \partial_{i} \theta \partial_{j}\left(u_{3}-\widetilde{u}_{3}\right) \\
& \left.\quad+\int_{D}\left(\widetilde{n}_{i j}^{\theta}-n_{i j}^{\theta}\right)(\widetilde{u}) \partial_{j} \widetilde{u}_{i}^{\prime}+\left(\tilde{m}_{i j}-m_{i j}\right)(\widetilde{u}) \partial_{i j}^{2} \widetilde{u}_{3}+\left(\widetilde{n}_{i j}^{\theta}-n_{i j}^{\theta}\right)(\widetilde{u}) \partial_{i} \theta \partial_{j} \widetilde{u}_{3}\right) .
\end{aligned}
$$


Now combining (5.27) and (5.32) immediately yields

$$
\begin{aligned}
& \frac{1}{1+\varepsilon_{*}} \int_{D}(\tilde{a}-a)\left|\nabla \cdot u^{\prime}+\nabla \theta \cdot \nabla u_{3}\right|^{2}+(\tilde{b}-b) \sum_{i j}\left|e_{i j}^{\theta}(u)\right|^{2} \\
& \quad+(\tilde{c}-c)\left|\Delta u_{3}\right|^{2}+(\tilde{d}-d) \sum_{i j}\left|\partial_{i j}^{2} u_{3}\right|^{2} \\
& \leq W-\widetilde{W}
\end{aligned}
$$

and we obtain (5.13).

Remark 5.3. It is tempting to estimate the size of $D$ without the a priori fatness condition (5.11) as in [2,7], and [21]. The important tool in these papers is a global doubling inequality. It seems possible to derive the size estimate without the fatness condition for the shallow shell system since we have derived local doubling inequalities (4.4). Like Theorem 4.13, to investigate this inverse problem, we actually need global doubling inequalities in terms of $\sum_{i j}\left|e_{i j}^{\theta}(u)\right|^{2}+\rho_{0}^{2}\left|\partial_{i j}^{2} u_{3}\right|^{2}$ instead of $\left|u^{\prime}\right|^{2}+\left|u_{3}\right|^{2}$. However, attempts to derive such global doubling inequalities were unsuccessful. The difficulty is due to the fact that $u^{\prime}$ and $u_{3}$ in (3.4) have different scalings.

\section{References}

[1] G. Aless Andrini and A. Morassi, Strong unique continuation for the Lamé system of elasticity, Comm. Partial Differential Equations 26 (2001), 1787-1810.

[2] G. Alessandrini, A. Morassi and E. Rosset, Detecting an inclusion in an elastic body by boundary measurements, SIAM J. Math. Anal. 33 (2002), 1247-1268.

[3] G. Alessandrini, A. Morassi and E. Rosset, Size estimates, In: "Inverse Problems: Theory and Applications" (Cortona/Pisa, 2002), Contemp. Math., Vol. 333, Amer. Math. Soc., Providence, RI, 2003, 1-33.

[4] G. Alessandrini, A. Morassi, E. Rosset and S. Vessella, On doubling inequalities for elliptic systems, J. Math. Anal. Appl. 357 (2009), 349-355.

[5] G. Aless Andrini, A. Morassi and E. Rosset, The linear constraints in Poincaré and Korn type inequalities, Forum Math. 20 (2008), 557-569.

[6] G. AlESSANDRINI and E. RosSET, The inverse conductivity problem with one measurement: bounds on the size of the unknown object, SIAM J. Appl. Math. 58 (1998), 10601071.

[7] G. Alessandrini, E. Rosset and J. K. Seo, Optimal size estimates for the inverse conductivity problem with one measurement, Proc. Amer. Mat. Soc. 128 (2000), 53-64.

[8] J. Bourgain and C. KenIG, On localization in the continuous Anderson-Bernoulli model in higher dimension, Invent. Math. 161 (2005), 1389-426.

[9] H. Donnelly and C. FefFerman, Nodal sets of eigenfunctions on Riemannian manifolds, Invent. Math. 93 (1988), 161-183.

[10] N. GaRofalO and F. H. Lin, Monotonicity properties of variational integrals, $A_{p}$ weights and unique continuation, Indiana Univ. Math. J. 35 (1986), 245-267.

[11] N. GAROFALO and F. H. LIN, Unique continuation for elliptic operators: a geometricvariational approach, Comm. Pure Appl. Math., 40 (1987), 347-366. 
[12] L. HöRmander, "The Analysis of Linear Partial Differential Operators”, Vol. 3, SpringerVerlag, Berlin/New York, 1985.

[13] V. IsAKov, "Inverse Problems for Partial Differential Equations", Second Edition, Springer, New York, 2006.

[14] I. KUKAVICA, Quantitative uniqueness for second order elliptic operators, Duke Math. J. 91 (1998), 225-240.

[15] C.L. Lin, Strong unique continuation for $m$-th powers of a Laplaceian operator with singular coefficients, Proc. Amer. Math. Soc. 135 (2007), 569-578.

[16] F. H. Lin, A uniqueness theorem for parabolic equations, Comm. Pure Appl. Math. $\mathbf{4 3}$ (1990), 127-136.

[17] C. L. Lin, S. NAGAYASU and J. N. WANG, Quantitative uniqueness for the power of the Laplacian with singular coefficients, Ann. Sc. Norm. Super. Pisa Cl. Sci. (5) 10 (2011), 513-529

[18] C. L. Lin, G. NAKAMURA and J. N. WANG, Quantitative uniqueness for second order elliptic operators with strongly singular coefficients, preprint.

[19] C. L. LIN, G. NAKAMURA and J. N. WANG, Optimal three-ball inequalities and quantitative uniqueness for the Lamé system with Lipschitz coefficients, arXiv:0901.4638 [math.AP]

[20] A. Morassi, E. Rosset and S. Vessella, Size estimates for inclusions in an elastic plate by boundary measurements, Indiana Univ. Math. J. 56 (2007), 2325-2384.

[21] A. Morassi, E. Rosset and S. Vessella, Detecting general inclusions in elastic plates, Inverse Problems 25 (2009).

Dipartimento di Matematica

Politecnico di Milano

Piazza Leonardo da Vinci, 32

20133 Milano, Italia

michele.dicristo@polimi.it

Department of Mathematics

NCTS National Cheng Kung University

Tainan 701, Taiwan

cllin2@mail.ncku.edu.tw

Department of Mathematics

NCTS (Taipei)

National Taiwan University

Taipei 106, Taiwan

jnwang@math.ntu.edu.tw 